\title{
Thermal Cycloisomerization of Putative Allenylpyridines for the Synthesis of Isoquinoline Derivatives
}

\author{
Alec E. Morrison ${ }^{\dagger}$, Jeremy J. Hrudka ${ }^{\dagger}$, and Gregory B. Dudley*, ${ }^{\dagger, \ddagger}$ \\ ${ }^{\dagger}$ Department of Chemistry and Biochemistry, Florida State University, Tallahassee, Florida, 32306 \\ ${ }^{\ddagger}$ C. Eugene Bennett Department of Chemistry, West Virginia University, Morgantown, WV, 26506, USA \\ Department of Chemistry and Biochemistry, Florida State University, Tallahassee, Florida, 32306-4390, \\ USA
}

\section{Supporting Information}

\section{Contents:}

I. General Information

II. Literature Preparation of Fragmentation/Olefination Precursors

III. Synthesis of phenylthio-enyne substrates

IV. Sonogashira coupling of aryl halides and 1,6-enynes

V. Synthesis of malonate-tethered diynyl-pyridine $\mathbf{1}$

VI. Synthesis of Isoquinolines (and Quinolines) via DBU-promoted Benzannulation...(S11)

VII. Synthesis of Brominated Isoquinolines

VIII. Site selective substitution of $\mathbf{1 3}$

IX. Synthesis of Pentacyclic Phenanthridine 28

X. Deuterium Labeling Experiments

XI. Copy of ${ }^{1} \mathrm{H}-\mathrm{NMR}$ and ${ }^{13} \mathrm{C}-\mathrm{NMR}$ Data.

XII. Absorption and Emission of 21, 23, and 28 .

XIII. X-ray Crystallography of $\mathbf{2 8}$ 


\section{General Information}

${ }^{1} \mathrm{H}-\mathrm{NMR}$ and ${ }^{13} \mathrm{CNMR}$ spectra were obtained on a 400 or $600 \mathrm{MHz}$ spectrometer using $\mathrm{CDCl}_{3}$ as the deuterated solvent. Chemical shifts are reported in parts per million (ppm) relative to residual $\mathrm{CHCl}_{3}$ (7.26 ppm for ${ }^{1} \mathrm{H}-\mathrm{NMR}$ and $77.0 \mathrm{ppm}$ for ${ }^{13} \mathrm{CNMR}$ ). Coupling constants (J) are reported in Hertz (Hz). IR spectra were recorded on an FT-IR spectrometer with diamond ATR accessory as thin film. Mass spectra were recorded using electrospray ionization (ESI) or atmospheric pressure chemical ionization (APCI). Melting points were taken using an electrothermal Mel-Temp (C) apparatus. All chemicals were used as received without further purification and all reactions were run under nitrogen atmosphere. Glassware was oven-dried prior to use and all purifications were performed by flash chromatography using silica gel with 40-63 micron particle size. Absorption spectra data were recorded on an Agilent 8453 UV-visible photo diode array spectrophotometer in a quartz cuvette with a path length of $1 \mathrm{~cm}$. Steady-state emission data were collected at room temperature using an Edinburgh FLS980 spectrometer. Samples were excited using light output from a housed $450 \mathrm{~W}$ Xe lamp passed through a single grating $(1800 \mathrm{l} / \mathrm{mm}, 250 \mathrm{~nm}$ blaze) Czerny-Turner monochromator and finally a $1 \mathrm{~nm}$ bandwidth slit. Emission from the sample was passed through a single grating $(1800 \mathrm{l} / \mathrm{mm}, 500 \mathrm{~nm}$ blaze) Czerny-Turner monochromator (1 $\mathrm{nm}$ bandwidth) and detected by a peltier-cooled Hamamatsu R928 photomultiplier tube.

\section{Literature Preparation of Fragmentation/Olefination Precursors}<smiles>CCCCOC1=CC(=O)CC(C)(C)C1</smiles>

\section{5,5-dimethyl-3-oxocyclohex-1-en-1-yl trifluoromethanesulfonate:}

To a suspension of 5,5-dimethyl-1,3-cyclohexanedione (1.38 g, $9.8 \mathrm{mmol}, 1$ equiv.) in dichloromethane $(50 \mathrm{~mL})$ was added pyridine $\left(1.59 \mathrm{~mL}, 19.6 \mathrm{mmol}, 2\right.$ equiv.). The resulting mixture was stirred at $-78{ }^{\circ} \mathrm{C}$ for 10 minutes before trifluoromethanesulfonic anhydride $(1.98 \mathrm{~mL}, 11.8 \mathrm{mmol}, 1.2$ equiv.) was added dropwise via syringe. The reaction was stirred at $-78{ }^{\circ} \mathrm{C}$ for 20 minutes, warmed to $0^{\circ} \mathrm{C}$ for 20 minutes, and room temperature for 30 minutes. When complete consumption of the starting dione was observed by TLC, the reaction was quenched using $1 \mathrm{M} \mathrm{HCl}$ solution $(20 \mathrm{~mL})$ and extracted 3 times with diethyl ether $(3 \times 10 \mathrm{~mL})$. The organic layers were combined and washed with aqueous $\mathrm{Na}_{2} \mathrm{CO}_{3}$ solution and water, dried over $\mathrm{Na}_{2} \mathrm{SO}_{4}$, filtered and concentrated by rotary evaporation. The residue was purified by flash column chromatography (eluent mixture: EtOAc/Hexane $=2 / 98$ ) to yield $2.54 \mathrm{~g}$ vinyl triflate in $95 \%$ yield as a colorless oil. Spectroscopic data was identical to the reported data from the literature. ${ }^{1}$

\footnotetext{
${ }^{1}$ Hoang, T. T.; Dudley, G. B. Org. Lett. 2013, 15, 4026
} 
<smiles>CCCCCCOC1=CC(O)CC(C)(C)C1</smiles>

\section{3-hydroxy-5,5-dimethylcyclohex-1-en-1-yl trifluoromethanesulfonate (5)}

To $40 \mathrm{~mL}$ THF solution of vinyl triflate (see above) $\left(2.67 \mathrm{~g}, 9.81 \mathrm{mmol}, 1\right.$ equiv.) at $-78{ }^{\circ} \mathrm{C}$ was slowly added $11.77 \mathrm{~mL}$ DIBAL-H (1.0 M solution in toluene, 1.2 equiv.). The reaction mixture was stirred at -78 ${ }^{\circ} \mathrm{C}$ for 10 minutes, warmed to $0{ }^{\circ} \mathrm{C}$ for 10 minutes, and room temperature for 30 minutes. The reaction was diluted with ether, cooled to $0{ }^{\circ} \mathrm{C}$ and quenched by adding $15 \% \mathrm{NaOH}$ and water. The mixture was stirred for 15 minutes until a gel formed, and $\mathrm{MgSO}_{4}$ was then added. After the addition of $\mathrm{MgSO}_{4}$, the mixture was stirred for an additional 15 minutes. Vacuum filtration and evaporation gave the crude vinylogous hemiacetal triflate 5. Purification flash column chromatography with gradient eluent from EtOAc/Hexane $=5 / 95$ to EtOAc/Hexane $=20 / 80$ yielded $2.61 \mathrm{~g}$ of $5(97 \%)$. Spectroscopic data was identical to the reported data from the literature. ${ }^{2}$<smiles>CCOP(=O)(CSc1ccccc1)OCC</smiles>

\section{diethyl ((phenylthio)methyl)phosphonate (6)}

To a round-bottom flask equipped with a reflux condenser and magnetic stir bar was added chloromethyl phenyl sulfide ( $0.63 \mathrm{~g}, 4 \mathrm{mmol}, 1$ equiv.) and triethyl phosphite (1.66 g, $10 \mathrm{mmol}, 2.5$ equiv.). The resulting mixture was heated at $150^{\circ} \mathrm{C}$ for 48 hours before being purified by Kugelrohr distillation to give diethyl phenylthiomethylphosphonate $(0.967 \mathrm{~g}, 93 \%)$ as colorless oil. Spectroscopic data was identical to the reported data. ${ }^{3}$<smiles>CCOP(=O)(OCC)C(C)[SnH]c1ccccc1</smiles>

\section{diethyl (1-(phenylthio)ethyl)phosphonate (7)}

To $4.22 \mathrm{~mL}$ THF solution of phosphonate $6\left(0.439 \mathrm{~g}, 1.69 \mathrm{mmol}, 1\right.$ equiv.) at $-78{ }^{\circ} \mathrm{C}$ was slowly added $1.16 \mathrm{~mL}$ of $n$-BuLi (1.6 M solution in hexanes, 1.1 equiv.). The reaction mixture was stirred at $-78{ }^{\circ} \mathrm{C}$ for 2 hours before iodomethane $(0.263 \mathrm{~g}, 1.87 \mathrm{mmol}, 1.1$ equiv. $)$ was added dropwise via syringe. The reaction was maintained at $-78{ }^{\circ} \mathrm{C}$ for an additional 30 minutes, warmed to $0{ }^{\circ} \mathrm{C}$ for 5 minutes, room temperature for 15 minutes, and heated in an oil bath at $45{ }^{\circ} \mathrm{C}$ for 18 hours. After 18 hours, the reaction mixture was cooled to room temperature and quenched with saturated $\mathrm{NH}_{4} \mathrm{Cl}$. The aqueous layer was extracted with diethyl ether 3 times and the combined organic layers were washed with saturated $\mathrm{NaCl}$, dried over $\mathrm{MgSO}_{4}$, and concentrated in vacuo. The crude product was purified by flash column

\footnotetext{
${ }^{2}$ Hoang, T. T.; Dudley, G. B. Org. Lett. 2013, 15, 4026

${ }^{3}$ Theobald, P. G.; Okamura, W. H. J. Org. Chem. 1990, 55, 741
} 
chromatography (eluent mixture: EtOAc/Hexane $=65 / 35)$ to give $7(0.338 \mathrm{~g}, 70 \%)$ as a colorless oil. Spectroscopic data was identical to the reported data. ${ }^{4}$

\section{Synthesis of Phenylthio-enyne Substrates}
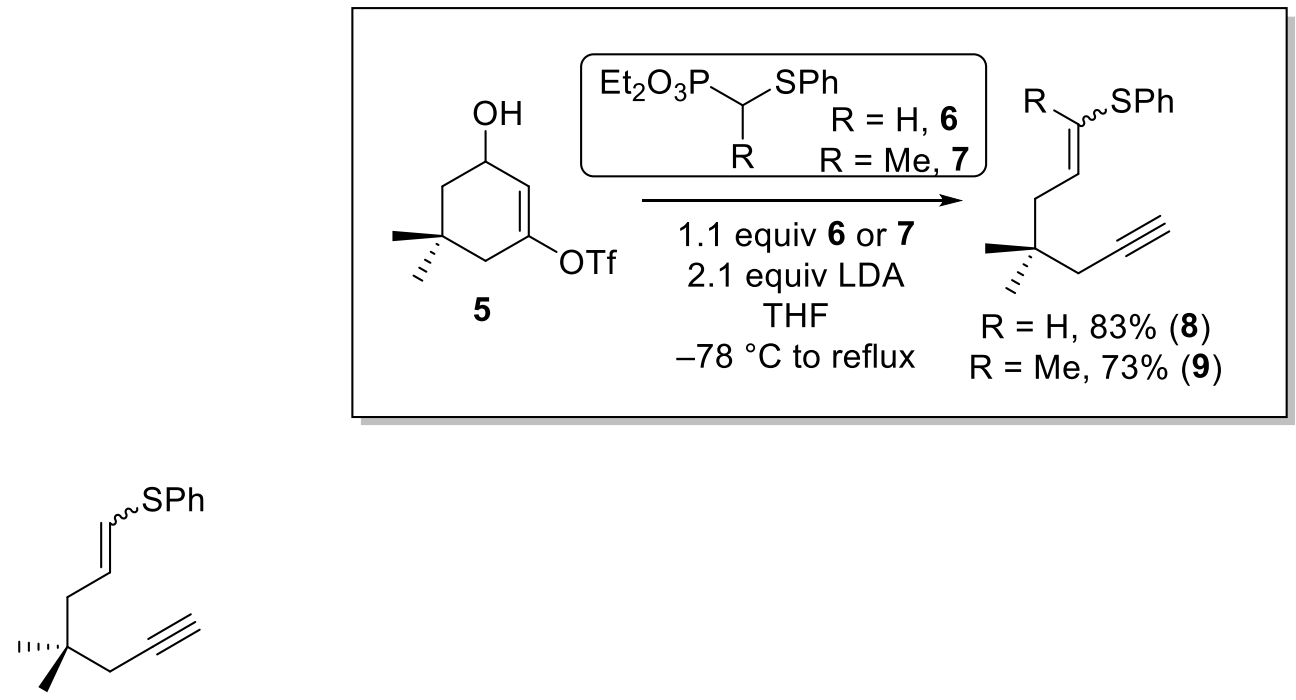

\section{(4,4-dimethylhept-1-en-6-yn-1-yl)(phenyl)sulfane (8)}

To $70 \mathrm{~mL}$ THF solution of diisopropylamine $(0.1 \mathrm{M})$ at $-78{ }^{\circ} \mathrm{C}$ was slowly added $4.15 \mathrm{~mL}$ of $n$-BuLi (1.6 $\mathrm{M}$ solution in hexanes, 2.1 equiv.). The mixture was stirred at $-78{ }^{\circ} \mathrm{C}$ for 10 minutes, warmed to $0{ }^{\circ} \mathrm{C}$ for 30 minutes, and then cooled to $-78^{\circ} \mathrm{C}$ before the addition of vinylogous hemiacetal triflate 5 . To the LDA solution (6.62 mmol, 2.1 equiv) at $-78{ }^{\circ} \mathrm{C}$ was added vinylogous hemiacetal triflate 5 ( $0.784 \mathrm{~g}, 2.86$ mmol, 1 equiv) and phosphonate $6(0.82 \mathrm{~g}, 3.15 \mathrm{mmol}, 1.1$ equiv) successively. The resulting mixture was stirred at $-78{ }^{\circ} \mathrm{C}$ for 10 minutes, warmed to $0{ }^{\circ} \mathrm{C}$ for 10 minutes, room temperature for 30 minutes, and heated in an oil bath at $60{ }^{\circ} \mathrm{C}$ for 2 hours. After 2 hours, the reaction mixture was cooled to room temperature and half-saturated $\mathrm{NH}_{4} \mathrm{Cl}$ was added to quench the reaction. The mixture was extracted with diethyl ether 3 times. The organic layers were combined and washed with water, dried over $\mathrm{MgSO}_{4}$ and concentrated. The residue was purified by flash column chromatography (eluent mixture: EtOAc/Hexane $=10 / 90)$ to give the desired enyne $8(0.547 \mathrm{~g}, 83 \%, E: Z=3.7: 1)$ as a colorless oil.

${ }^{1}$ HNMR trans isomer $\left(600 M H z, \mathbf{C D C l}_{3}\right): \delta 7.35-7.28(\mathrm{~m}, 5 \mathrm{H}), 6.20(\mathrm{dt}, \mathrm{J}=14.82,0.84 \mathrm{~Hz}, 1 \mathrm{H}), 5.97$ $(\mathrm{dt}, \mathrm{J}=14.82,7.80 \mathrm{~Hz}, 1 \mathrm{H}), 2.19(\mathrm{dd}, \mathrm{J}=7.74,1.02 \mathrm{~Hz}, 2 \mathrm{H}), 2.10(\mathrm{~d}, \mathrm{~J}=2.64,2 \mathrm{H}), 2.01(\mathrm{t}, \mathrm{J}=2.64$, $1 \mathrm{H}), 1.00(\mathrm{~s}, 6 \mathrm{H}) \mathrm{ppm}$;

${ }^{1} \mathbf{H N M R}$ cis isomer $\left(\mathbf{6 0 0 M H z}, \mathbf{C D C l}_{3}\right): \delta$ 7.22-7.18 $(\mathrm{m}, 5 \mathrm{H}), 6.32(\mathrm{dt}, \mathbf{J}=9.42,1.08 \mathrm{~Hz}, 1 \mathrm{H}), 5.86(\mathrm{dt}, \mathbf{J}$ $=9.36,7.74 \mathrm{~Hz}, 1 \mathrm{H}), 2.30(\mathrm{dd}, \mathrm{J}=7.68,1.14 \mathrm{~Hz}, 2 \mathrm{H}), 2.15(\mathrm{~d}, \mathrm{~J}=2.64,2 \mathrm{H}), 2.01(\mathrm{t}, \mathrm{J}=2.64,1 \mathrm{H}), 1.04$ (s, 6H) ppm;

${ }^{13} \mathbf{C N M R}$ of mixture $\boldsymbol{E}$ and $\boldsymbol{Z}\left(\mathbf{6 0 0 M H z}, \mathbf{C D C l}_{3}\right): \delta 136.38,136.33,132.81,129.36,128.98,128.95$, $128.64,126.25,126.19,125.39,123.96,82.30,82.19,70.24,70.18,44.54,40.61,34.55,34.07,31.63$, 31.38, 26.65, $26.52 \mathrm{ppm}$;

\footnotetext{
${ }^{4}$ Theobald, P. G.; Okamura, W. H. J. Org. Chem. 1990, 55, 741
} 
IR: $v_{\max } 3300,3067,3016,2958,2926,2116,1583,1479,1439,1386,1367,1090,1024,952,737,689$.

HRMS (APCI) calcd for $\mathrm{C}_{15} \mathrm{H}_{19} \mathrm{~S}^{+}\left[(\mathrm{M}+\mathrm{H})^{+}\right]: 231.12020$, found 231.11998<smiles>C#CCC(C)(C)CC=C(C)[SbH2]c1ccccc1</smiles>

\section{(5,5-dimethyloct-2-en-7-yn-2-yl)(phenyl)sulfane (9)}

Following the same procedure for 8 , phosphonate 7 ( $0.25 \mathrm{~g}, 0.89 \mathrm{mmol}, 2.1$ equiv.) gave enyne 9 ( 0.145 $\mathrm{g}, 73 \%, E: Z=2.7: 1)$ as a colorless oil.

${ }^{1}$ HNMR trans isomer $\left(\mathbf{6 0 0 M H z}, \mathbf{C D C l}_{3}\right): \delta 7.33-7.28(\mathrm{~m}, 5 \mathrm{H}), 5.89(\mathrm{td}, \mathrm{J}=7.89,1.32 \mathrm{~Hz}, 1 \mathrm{H}), 2.15(\mathrm{~d}$, $\mathrm{J}=7.92 \mathrm{~Hz}, 2 \mathrm{H}), 2.10(\mathrm{~d}, \mathrm{~J}=2.64 \mathrm{~Hz}, 2 \mathrm{H}), 2.00(\mathrm{t}, \mathrm{J}=2.64 \mathrm{~Hz}, 1 \mathrm{H}), 1.90(\mathrm{~s}, 3 \mathrm{H}), 1.00(\mathrm{~s}, 6 \mathrm{H}) \mathrm{ppm}$;

${ }^{1}$ HNMR cis isomer $\left(\mathbf{6 0 0 M H z}, \mathbf{C D C l}_{3}\right): \delta 7.22-7.19(\mathrm{~m}, 5 \mathrm{H}), 5.84(\mathrm{td}, \mathrm{J}=7.53,1.08 \mathrm{~Hz}, 1 \mathrm{H}), 2.38(\mathrm{dd}, \mathrm{J}$ = 7.56, $0.72 \mathrm{~Hz}, 2 \mathrm{H}), 2.13(\mathrm{~d}, \mathrm{~J}=2.64 \mathrm{~Hz}, 2 \mathrm{H}), 2.00(\mathrm{t}, \mathrm{J}=2.64 \mathrm{~Hz}, 1 \mathrm{H}), 1.92(\mathrm{~d}, \mathrm{~J}=1.02 \mathrm{~Hz}, 3 \mathrm{H}), 1.02$ (s, 6H) ppm;

${ }^{13} \mathbf{C N M R}$ of mixture $\boldsymbol{E}$ and $\boldsymbol{Z}\left(\mathbf{6 0 0 M H z}, \mathbf{C D C l}_{\mathbf{3}}\right): \delta 135.19,134.77,131.80,131.39,131.19,130.93$, $130.48,130.42$, 128.93, 128.81, 126.52, 126.31, 82.45, 82.33, 70.17, 70.12, 41.37, 40.39, 34.68, 34.44, $31.63,31.51,26.64,24.61,18.25 \mathrm{ppm}$

IR: $v_{\max } 3301,2956,2868,1711,1580,1477,1439,1230,1023,821,739,688$.

HRMS (APCI) calcd for $\mathrm{C}_{16} \mathrm{H}_{19} \mathrm{~S}^{+}\left[(\mathrm{M}+\mathrm{H})^{+}\right]$: 243.12020, found 243.12000

\section{Sonogashira coupling of aryl halides and 1,6-enynes}

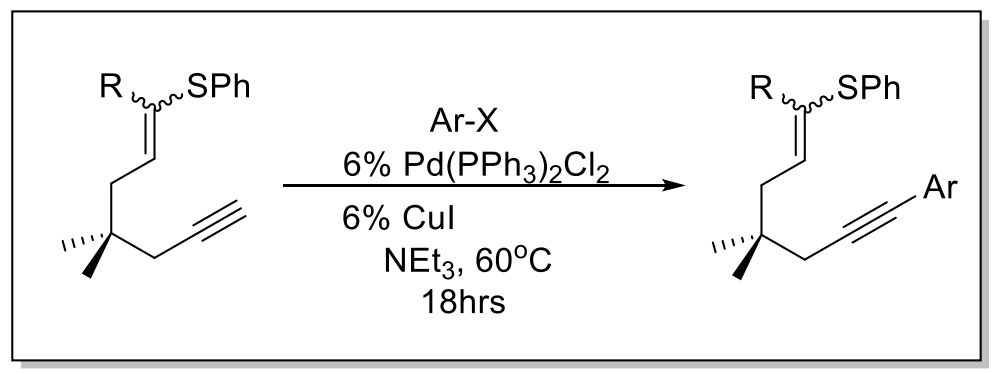

\section{General Procedure A:}

An oven-dried flask under the flow of $\mathrm{N}_{2}$ was charged with aryl halide (1 equiv.), $\mathrm{Pd}\left(\mathrm{PPh}_{3}\right)_{2} \mathrm{Cl}_{2}(0.06$ equiv.), and $\mathrm{CuI}$ (0.06 equiv.). The flask was further vacuumed and filled with $\mathrm{N}_{2} 3$ times before the addition of degassed $\mathrm{NEt}_{3}(0.1 \mathrm{M})$. The mixture was stirred for 5 minutes before slow, dropwise addition of enyne 8 or 9 (1.13 equiv.) and heating to $60^{\circ} \mathrm{C}$ for 24 hours. After 24 hours the reaction mixture was 
passed through a plug of silica gel and celite using $80 \%$ EtOAc in hexanes as the eluent and concentrated in vacuo. The crude product was purified by flash column chromatography (eluent mixture: EtOAc/Hexane $=10 / 90$ ) to give the desired product.<smiles>CC(C)(CC#Cc1ccncc1)C/C=C/[SbH]</smiles>

\section{4-(4,4-dimethyl-7-(phenylthio)hept-6-en-1-yn-1-yl)pyridine (3)}

Following the general procedure A, 4-iodopyridine $(0.236 \mathrm{~g}, 1.15 \mathrm{mmol})$ and enyne $\mathbf{8}(0.30 \mathrm{~g}, 1.30 \mathrm{mmol})$ gave $3(0.308 \mathrm{~g}, 87 \%, E: Z=2.5: 1)$ as a yellow oil.

${ }^{1}$ HNMR trans isomer $\left(\mathbf{6 0 0 M H z}, \mathbf{C D C l}_{3}\right): \delta 8.52(\mathrm{~d}, \mathrm{~J}=2.94 \mathrm{~Hz}, 2 \mathrm{H}), 7.34-7.24(\mathrm{~m}, 7 \mathrm{H}), 6.22(\mathrm{dt}, \mathrm{J}=$ 14.94, $0.86 \mathrm{~Hz}, 1 \mathrm{H}), 6.00(\mathrm{dt}, \mathrm{J}=14.88,7.8 \mathrm{~Hz}, 1 \mathrm{H}), 2.34(\mathrm{~s}, 2 \mathrm{H}), 2.23(\mathrm{dd}, \mathrm{J}=7.74,0.86 \mathrm{~Hz}, 2 \mathrm{H}), 1.06$ (s, 6H) ppm;

${ }^{1}$ HNMR cis isomer $\left(\mathbf{6 0 0 M H z}, \mathbf{C D C l}_{3}\right): \delta 8.51(\mathrm{~d}, \mathrm{~J}=5.94 \mathrm{~Hz}, 2 \mathrm{H}), 7.25-7.18(\mathrm{~m}, 7 \mathrm{H}), 6.35(\mathrm{dt}, \mathrm{J}=9.24$, $1.14 \mathrm{~Hz}, 1 \mathrm{H}), 5.89$ (dt, J = 9.30, $7.68 \mathrm{~Hz}, 1 \mathrm{H}), 2.38(\mathrm{~s}, 2 \mathrm{H}), 2.36(\mathrm{dd}, \mathrm{J}=8.46,1.14 \mathrm{~Hz}, 2 \mathrm{H}), 1.10(\mathrm{~s}, 6 \mathrm{H})$ ppm;

${ }^{13} \mathbf{C N M R}$ of mixture $\boldsymbol{E}$ and $\boldsymbol{Z}\left(\mathbf{6 0 0 M H z}, \mathbf{C D C l}_{3}\right): \delta 149.63,149.57,136.26,136.19,132.38,132.27$, $132.15,129.15,129.02,128.87,128.66,126.29,125.80,125.62,124.31,93.87,93.66,80.47,80.42$, $44.85,40.70,35.15,34.6832 .54,32.31,26.91,26.83 \mathrm{ppm}$;

IR: $v_{\max } 3079,3019,2958,2224,1715,1592,1538,1478,1278,1213,1156,988,951,819,737,689$.

HRMS (ESI+) calcd for $\mathrm{C}_{20} \mathrm{H}_{22} \mathrm{NS}^{+}\left[(\mathrm{M}+\mathrm{H})^{+}\right]$: 308.14729, found 308.14826.<smiles>CC(=CCC(C)(C)CC#Cc1ccncc1)Sc1ccccc1</smiles>

\section{4-(4,4-dimethyl-7-(phenylthio)oct-6-en-1-yn-1-yl)pyridine (10)}

Following the general procedure A, 4-iodopyridine $(0.072 \mathrm{~g}, 0.353 \mathrm{mmol})$ and enyne $9(0.110 \mathrm{~g}, 0.45$ $\mathrm{mmol})$ gave $10(0.096 \mathrm{~g}, 85 \%, E: Z=3.85: 1)$ as a colorless oil.

${ }^{1}$ HNMR $\boldsymbol{E}$ isomer $\left(\mathbf{6 0 0 M H z}, \mathbf{C D C l}_{3}\right): \delta 8.53(\mathrm{~d}, \mathrm{~J}=3.48 \mathrm{~Hz}, 2 \mathrm{H}), 7.52-7.18(\mathrm{~m}, 7 \mathrm{H}), 5.91(\mathrm{td}, \mathrm{J}=7.92$, $1.32 \mathrm{~Hz}, 1 \mathrm{H}), 2.34(\mathrm{~s}, 2 \mathrm{H}), 2.20(\mathrm{~d}, \mathrm{~J}=7.80 \mathrm{~Hz}, 2 \mathrm{H}), 1.91(\mathrm{~d}, \mathrm{~J}=0.92 \mathrm{~Hz}, 3 \mathrm{H}), 1.06(\mathrm{~s}, 6 \mathrm{H}) \mathrm{ppm}$

${ }^{1}$ HNMR $Z$ isomer $\left(600 M H z, \mathbf{C D C l}_{3}\right): \delta 8.53(\mathrm{~d}, \mathrm{~J}=3.48 \mathrm{~Hz}, 2 \mathrm{H}), 7.52-7.18(\mathrm{~m}, 7 \mathrm{H}), 5.87(\mathrm{td}, \mathrm{J}=7.50$, $1.28 \mathrm{~Hz}, 1 \mathrm{H}), 2.45(\mathrm{~d}, \mathrm{~J}=6.40 \mathrm{~Hz}, 2 \mathrm{H}), 2.36(\mathrm{~s}, 2 \mathrm{H}), 1.94(\mathrm{~d}, \mathrm{~J}=1.16 \mathrm{~Hz}, 3 \mathrm{H}), 1.08$ (s, 6H) ppm 
${ }^{13} \mathrm{CNMR}$ of mixture $\boldsymbol{E}$ and $\boldsymbol{Z}\left(\mathbf{6 0 0 M H z}, \mathbf{C D C l}_{\mathbf{3}}\right): \delta$ 149. 57, 149.48, 135.03, 134.68, 132.37, 132.24, $131.47,131.33,131.27,131.16,130.54,130.40,128.95,128.84,126.63,126.35,125.80,94.10,93.89$, $80.38,41.46,40.61,35.26,35.04,32.59,32.41,26.93,24.63,18.26 \mathrm{ppm}$

IR: $v_{\max } 3067,3031,2958,2926,2225,1593,1530,1475,1439,1262,1213,1168,1024,819,740,691$.

HRMS (ESI+) calcd for $\mathrm{C}_{21} \mathrm{H}_{24} \mathrm{NS}^{+}\left[(\mathrm{M}+\mathrm{H})^{+}\right]$: 322.16294 , found 322.16388

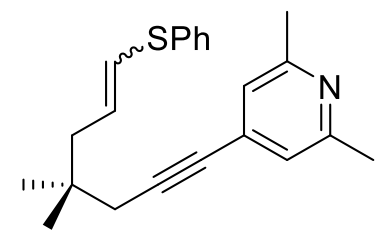

4-(4,4-dimethyl-7-(phenylthio)hept-6-en-1-yn-1-yl)-2,6-dimethylpyridine (12)

Following the general procedure A, 4-bromo-2,6-dimethylpyridine $(0.20 \mathrm{~g}, 1.075 \mathrm{mmol})$ and enyne 8 $(0.297 \mathrm{~g}, 1.29 \mathrm{mmol})$ gave $\mathbf{1 2}(0.335 \mathrm{~g}, 93 \%, E: Z=4: 1)$ as a brown oil.

${ }^{1}$ HNMR trans isomer $\left(\mathbf{6 0 0 M H z}, \mathbf{C D C l}_{3}\right): \delta 7.34-7.27(\mathrm{~m}, 5 \mathrm{H}), 6.94(\mathrm{~s}, 2 \mathrm{H}), 6.22(\mathrm{dt}, \mathrm{J}=14.94,0.78 \mathrm{~Hz}$, $1 \mathrm{H}), 6.00(\mathrm{dt}, \mathrm{J}=14.82,7.8 \mathrm{~Hz}, 1 \mathrm{H}), 2.47(\mathrm{~s}, 6 \mathrm{H}), 2.31(\mathrm{~s}, 2 \mathrm{H}), 2.22(\mathrm{dd}, \mathrm{J}=5.97,0.78 \mathrm{~Hz}, 2 \mathrm{H}), 1.05(\mathrm{~s}$, 6H) ppm;

${ }^{1}$ HNMR cis isomer $\left(\mathbf{6 0 0 M H z}, \mathbf{C D C l}_{3}\right): \delta 7.21-7.18(\mathrm{~m}, 5 \mathrm{H}), 6.94(\mathrm{~s}, 2 \mathrm{H}), 6.35(\mathrm{dt}, \mathrm{J}=9.3,1.02 \mathrm{~Hz}, 1 \mathrm{H})$, $5.89(\mathrm{dt}, \mathrm{J}=9.3,7.62 \mathrm{~Hz}, 1 \mathrm{H}), 2.46(\mathrm{~s}, 6 \mathrm{H}), 2.35(\mathrm{~s}, 2 \mathrm{H}), 2.35(\mathrm{dd}, \mathrm{J}=8.58,1.02 \mathrm{~Hz}, 2 \mathrm{H}), 1.09(\mathrm{~s}, 6 \mathrm{H})$ ppm;

${ }^{13} \mathbf{C N M R}$ of mixture $\boldsymbol{E}$ and $\boldsymbol{Z}\left(\mathbf{6 0 0 M H z}, \mathbf{C D C l}_{3}\right): \delta 157.67,157.59,136.33,136.24,132.49,132.44$, $129.29,129.00,128.85,128.68,126.26,125.50,124.24,122.34,122.30,92.28,80.89,80.82,44.83$, $40.68,35.13,34.68,32.52,32.31,26.92,26.83,24.29 \mathrm{ppm}$;

IR: $v_{\max } 3059,3007,2958,2924,2246,2220,1597,1550,1478,1214,1090,1024,950,860,737,689$.

HRMS (ESI+) calcd for $\mathrm{C}_{22} \mathrm{H}_{26} \mathrm{NS}^{+}\left[(\mathrm{M}+\mathrm{H})^{+}\right]: 336.17859$, found 336.17789

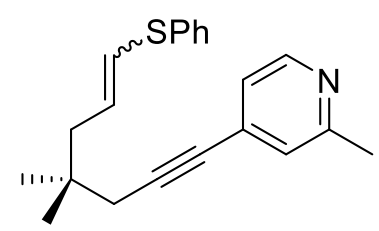

\section{4-(4,4-dimethyl-7-(phenylthio)hept-6-en-1-yn-1-yl)-2-methylpyridine (14)}

Following the general procedure A, 4-bromo-2-methylpyridine $(0.081 \mathrm{~g}, 0.47 \mathrm{mmol}, 1.13 \mathrm{equiv}$. $)$ and enyne 8 (0.096 g, $0.42 \mathrm{mmol}, 1$ equiv.) gave $14(0.095 \mathrm{~g}, 71 \%, E: Z=2.5: 1)$ as a brown oil. 
${ }^{1}$ HNMR trans isomer (400MHz, $\left.\mathbf{C D C l}_{3}\right): \delta 8.41(\mathrm{~d}, \mathrm{~J}=4.96 \mathrm{~Hz}, 1 \mathrm{H}), 7.35-7.17(\mathrm{~m}, 5 \mathrm{H}), 7.13(\mathrm{~s}, 1 \mathrm{H})$, $7.06(\mathrm{~d}, \mathrm{~J}=4.08 \mathrm{~Hz}, 1 \mathrm{H}), 6.23(\mathrm{dt}, \mathrm{J}=14.88,0.92 \mathrm{~Hz}, 1 \mathrm{H}), 6.00(\mathrm{dt}, \mathrm{J}=14.84,7.76,1 \mathrm{H}), 2.51(\mathrm{~s}, 3 \mathrm{H})$, $2.33(\mathrm{~s}, 2 \mathrm{H}), 2.23(\mathrm{dd}, \mathrm{J}=7.76,0.96 \mathrm{~Hz}, 2 \mathrm{H}), 1.06(\mathrm{~s}, 6 \mathrm{H}) \mathrm{ppm}$

${ }^{1}$ HNMR trans isomer (400MHz, $\left.\mathbf{C D C l}_{3}\right): \delta 8.40(\mathrm{~d}, \mathrm{~J}=4.6 \mathrm{~Hz}, 1 \mathrm{H}), 7.35-7.17(\mathrm{~m}, 5 \mathrm{H}), 7.13(\mathrm{~s}, 1 \mathrm{H})$, $7.06(\mathrm{~d}, \mathrm{~J}=4.08 \mathrm{~Hz}, 1 \mathrm{H}), 6.35(\mathrm{dt}, \mathrm{J}=9.32,1.14 \mathrm{~Hz}, 1 \mathrm{H}), 5.89(\mathrm{dt}, \mathrm{J}=9.32,7.68 \mathrm{~Hz}, 1 \mathrm{H}), 2.50(\mathrm{~s}, 3 \mathrm{H})$, 2.37 (s, 2H), 2.36 (dd, J = 7.67, $1.16 \mathrm{~Hz}, 2 \mathrm{H}), 1.09$ (s, 1H) ppm

${ }^{13} \mathbf{C N M R}$ of mixture $\boldsymbol{E}$ and $\boldsymbol{Z}\left(\mathbf{6 0 0 M H z}, \mathbf{C D C l}_{3}\right): 158.34,158.26,149.00,148.94,136.31,136.23$, $132.39,132.29,129.20,129.01,128.86,128.69$, 126.27, 125.58, 125.34, 125.30, 124.31, 122.85, 122.83, $93.13,92.92,80.70,80.65,44.85,40.85,35.13,34.67,32.53,32.31,29.72,29.38,26.91,26.84 \mathrm{ppm}$

IR: $v_{\max } 3067,3020,2958,2926,2234,1598,1536,1479,1439,1386,1283,1090,1024,950,833,737$, 689.

HRMS (ESI+) calcd for $\mathrm{C}_{21} \mathrm{H}_{24} \mathrm{NS}^{+}\left[(\mathrm{M}+\mathrm{H})^{+}\right]: 322.16294$, found 322.16272

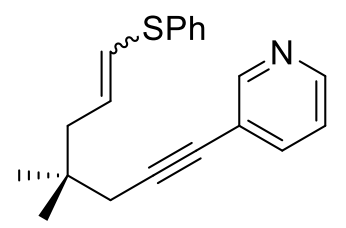

\section{3-(4,4-dimethyl-7-(phenylthio)hept-6-en-1-yn-1-yl)pyridine (16)}

Following the general procedure A, 3-iodopyridine ( $0.093 \mathrm{~g}, 0.45 \mathrm{mmol}, 1.13$ equiv.) and enyne 8 ( 0.092 $\mathrm{g}, 0.40 \mathrm{mmol}, 1$ equiv. $)$ gave $\mathbf{1 6}(0.105 \mathrm{~g}, 85 \%, E: Z=3.3: 1)$ as a light-brown oil.

${ }^{1}$ HNMR trans isomer (600MHz, $\left.\mathbf{C D C l}_{3}\right): \delta 8.63(\mathrm{~s}, 1 \mathrm{H}), 8.49(\mathrm{~d}, \mathrm{~J}=3.84 \mathrm{~Hz}, 1 \mathrm{H}), 7.66(\mathrm{~d}, \mathrm{~J}=7.84 \mathrm{~Hz}$, $1 \mathrm{H}), 7.35-7.27(\mathrm{~m}, 5 \mathrm{H}), 7.22-7.17(\mathrm{~m}, 1 \mathrm{H}), 6.23(\mathrm{~d}, \mathrm{~J}=14.88 \mathrm{~Hz}, 1 \mathrm{H}), 6.01(\mathrm{dt}, \mathrm{J}=14.84,7.76 \mathrm{~Hz}, 1 \mathrm{H})$, $2.34(\mathrm{~s}, 2 \mathrm{H}), 2.23(\mathrm{~d}, \mathrm{~J}=7.44 \mathrm{~Hz}, 2 \mathrm{H}), 1.06(\mathrm{~s}, 6 \mathrm{H}) \mathrm{ppm}$

${ }^{1}$ HNMR cis isomer $\left(\mathbf{6 0 0 M H z}, \mathbf{C D C l}_{3}\right): \delta 8.63(\mathrm{~s}, 1 \mathrm{H}), 8.49(\mathrm{~d}, \mathrm{~J}=3.84 \mathrm{~Hz}, 1 \mathrm{H}), 7.67(\mathrm{~d}, \mathrm{~J}=7.84 \mathrm{~Hz}$, $1 \mathrm{H}), 7.35-7.27(\mathrm{~m}, 1 \mathrm{H}), 7.22-7.17(\mathrm{~m}, 5 \mathrm{H}), 6.35(\mathrm{~d}, \mathrm{~J}=9.32 \mathrm{~Hz}, 1 \mathrm{H}), 5.90(\mathrm{dt}, \mathrm{J}=9.32,7.68 \mathrm{~Hz}, 1 \mathrm{H})$, $2.38(\mathrm{~s}, 2 \mathrm{H}), 2.36(\mathrm{~d}, \mathrm{~J}=7.72 \mathrm{~Hz}, 2 \mathrm{H}), 1.10(\mathrm{~s}, 6 \mathrm{H}) \mathrm{ppm}$

${ }^{13} \mathbf{C N M R}$ of mixture $\boldsymbol{E}$ and $\boldsymbol{Z}\left(\mathbf{6 0 0 M H z}, \mathbf{C D C l}_{3}\right): \delta 152.36,152.34,148.03,147.93,138.47,138.44$, $136.32,136.24,132.60,129.29,129.00,128.91,128.67,126.25,125.53,124.18,122.92,121.05,91.92$, $91.73,79.44,79.38,44.85,40.72,35.11,34.65,32.56,32.35,29.71,26.90,26.82 \mathrm{ppm}$

IR: $v_{\max } 3027,2958,2926,2222,1583,1560,1476,1439,1406,1184,1096,1023,950,802,737,704$, 689.

HRMS (ESI+) calcd for $\mathrm{C}_{20} \mathrm{H}_{22} \mathrm{NS}^{+}\left[(\mathrm{M}+\mathrm{H})^{+}\right]: 308.14729$, found 308.14811 
<smiles>CC(C)(CC#Cc1ccncc1Cl)C/C=C/[SbH]</smiles>

\section{3-chloro-4-(4,4-dimethyl-7-(phenylthio)hept-6-en-1-yn-1-yl)pyridine (18)}

Following the general procedure A, 3-chloro-4-iodopyridine ( $0.150 \mathrm{~g}, 0.626 \mathrm{mmol}, 1$ equiv) and enyne 8 (0.172 g, $0.75 \mathrm{mmol}, 1.2$ equiv.) gave $18(0.197 \mathrm{~g}, 92 \%, E: Z=3: 1)$ as a yellow oil.

${ }^{1}$ HNMR trans isomer $\left(\mathbf{6 0 0 M H z}, \mathbf{C D C l}_{3}\right): \delta 8.59(\mathrm{~s}, 1 \mathrm{H}), 8.39(\mathrm{~d}, \mathrm{~J}=4.98 \mathrm{~Hz}, 1 \mathrm{H}), 7.34-7.27(\mathrm{~m}, 6 \mathrm{H})$, $6.25(\mathrm{dt}, \mathrm{J}=14.88,0.85 \mathrm{~Hz}, 1 \mathrm{H}), 6.00(\mathrm{dt}, \mathrm{J}=14.82,7.8 \mathrm{~Hz}, 1 \mathrm{H}), 2.40(\mathrm{~s}, 2 \mathrm{H}), 2.27$ (dd, J = 7.77, 0.84 $\mathrm{Hz}, 2 \mathrm{H}), 1.09$ (s, 6H) ppm;

${ }^{1}$ HNMR cis isomer $\left(\mathbf{6 0 0 M H z}, \mathbf{C D C l}_{3}\right): \delta 8.58(\mathrm{~s}, 1 \mathrm{H}), 8.37(\mathrm{~d}, \mathrm{~J}=4.98 \mathrm{~Hz}, 1 \mathrm{H}), 7.21-7.18(\mathrm{~m}, 6 \mathrm{H}), 6.35$ (dt, J = 9.36, $1.02 \mathrm{~Hz}, 1 \mathrm{H}), 5.89(\mathrm{dt}, \mathrm{J}=9.36,7.74 \mathrm{~Hz}, 1 \mathrm{H}), 2.45$ (s, 2H), 2.38 (dd, J = 7.74, $1.02 \mathrm{~Hz}, 2 \mathrm{H})$, $1.13(\mathrm{~s}, 6 \mathrm{H}) \mathrm{ppm}$;

${ }^{13} \mathbf{C N M R}$ of mixture $\boldsymbol{E}$ and $\boldsymbol{Z}\left(\mathbf{6 0 0 M H z}, \mathbf{C D C l}_{3}\right): \delta 149.23,149.18,147.18,147.12,136.26,136.15$, 133.02, 132.21, 131.57, 131.47, 129.07, 129.00, 128.90, 128.71, 126.65, 126.60, 126.28, 125.73, 124.44, $99.78,99.59,77.63,77.59,44.73,40.77,35.28,34.81,32.79,32.50,26.93,26.82 \mathrm{ppm}$;

IR: $v_{\max } 3056,3020,2958,2925,2227,1726,1578,1470,1397,1167,1100,1024,951,832,736,689$.

HRMS (ESI+) calcd for $\mathrm{C}_{20} \mathrm{H}_{21} \mathrm{ClNS}^{+}\left[(\mathrm{M}+\mathrm{H})^{+}\right]$: 342.10832, found 342.10831<smiles>CC(C)(CC#Cc1ccnc2ccccc12)C/C=C/c1ccccc1</smiles>

\section{4-(4,4-dimethyl-7-(phenylthio)hept-6-en-1-yn-1-yl)quinolone (20)}

Following the general procedure A, 4-bromoquinoline $(0.084 \mathrm{~g}, 0.404 \mathrm{mmol}, 1.08$ equiv) and enyne 8 (0.086 g, $0.373 \mathrm{mmol}, 1$ equiv.) gave $20(0.090 \mathrm{~g}, 68 \%, E: Z=1.56: 1)$ as a yellow oil.

${ }^{1}$ HNMR trans isomer (400MHz, $\left.\mathbf{C D C l}_{3}\right): \delta 8.83(\mathrm{~d}, \mathrm{~J}=4.24 \mathrm{~Hz}, 1 \mathrm{H}), 8.26(\mathrm{dd}, \mathrm{J}=8.32,0.96 \mathrm{~Hz}, 1 \mathrm{H})$, $8.10(\mathrm{~d}, \mathrm{~J}=8.24 \mathrm{~Hz}, 1 \mathrm{H}), 7.75-7.71(\mathrm{~m}, 1 \mathrm{H}), 7.59-7.55(\mathrm{~m}, 1 \mathrm{H}), 7.44(\mathrm{~d}, \mathrm{~J}=4.44,1 \mathrm{H}), 7.35-7.27(\mathrm{~m}$, $5 \mathrm{H}), 6.26(\mathrm{dt}, \mathrm{J}=14.84,0.92 \mathrm{~Hz}, 1 \mathrm{H}), 6.04(\mathrm{dt}, \mathrm{J}=14.84,7.72 \mathrm{~Hz}, 1 \mathrm{H}) 2.51(\mathrm{~s}, 2 \mathrm{H}), 2.31(\mathrm{dd}, \mathrm{J}=7.72$, $0.92 \mathrm{~Hz}, 2 \mathrm{H}), 1.14(\mathrm{~s}, 6 \mathrm{H}) \mathrm{ppm}$

${ }^{1} \mathbf{H N M R}$ cis isomer (400MHz, $\left.\mathbf{C D C l}_{3}\right): \delta 8.82(\mathrm{~d}, \mathrm{~J}=4.04 \mathrm{~Hz}, 1 \mathrm{H}), 8.30(\mathrm{dd}, \mathrm{J}=8.30,0.96 \mathrm{~Hz}, 1 \mathrm{H})$, 8.09 (d, J = 8.44 Hz, 1H), 7.74-7.70 (m, 1H), 7.59-7.55 (m, 1H), $7.45(\mathrm{~d}, \mathrm{~J}=4.44,1 \mathrm{H}), 7.27-7.17(\mathrm{~m}$, $5 \mathrm{H}), 6.38(\mathrm{dt}, \mathrm{J}=9.36,1.12 \mathrm{~Hz}, 1 \mathrm{H}), 5.93(\mathrm{dt}, \mathrm{J}=9.36,7.68 \mathrm{~Hz}, 1 \mathrm{H}) 2.55(\mathrm{~s}, 2 \mathrm{H}), 2.44(\mathrm{dd}, \mathrm{J}=7.66,1.12$ $\mathrm{Hz}, 2 \mathrm{H}), 1.18(\mathrm{~s}, 6 \mathrm{H}) \mathrm{ppm}$ 
${ }^{13} \mathbf{C N M R}$ of mixture $\boldsymbol{E}$ and $\boldsymbol{Z}\left(\mathbf{6 0 0 M H z}, \mathbf{C D C l}_{3}\right): \delta 149.76,148.09,148.07,136.23,136.12,132.15,130$. $69,130.55,129.81,129.75,129.72,129.02,128.93,128.76,128.18,127.04,126.98,126.32,126.17$, $125.99,125.83,124.54,123.81,98.63,98.43,78.79,78.77,44.98,40.88,35.31,34.85,33.01,32.71$, $27.04,26.95 \mathrm{ppm}$

IR: $v_{\max } 3058,2956,2925,2228,1718,1683,1614,1578,1504,1388,1156,1023,896,761,739,689$.

HRMS (ESI+) calcd for $\mathrm{C}_{24} \mathrm{H}_{24} \mathrm{NS}^{+}\left[(\mathrm{M}+\mathrm{H})^{+}\right]: 358.16294$, found 358.16297

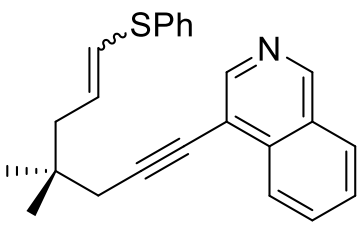

4-(4,4-dimethyl-7-(phenylthio)hept-6-en-1-yn-1-yl)isoquinoline (22)

Following the general procedure A, 4-bromoisoquinoline ( $0.082 \mathrm{~g}, 0.392 \mathrm{mmol}, 1.13$ equiv) and enyne 8 (0.080 g, $0.347 \mathrm{mmol}, 1$ equiv.) gave $22(0.109 \mathrm{~g}, 88 \%, E: Z=3.3: 1)$ as a yellow oil.

${ }^{1}$ HNMR trans isomer (600MHz, $\left.\mathbf{C D C l}_{3}\right): \delta 9.16(\mathrm{~s}, 1 \mathrm{H}), 8.65(\mathrm{~s}, 1 \mathrm{H}), 8.22(\mathrm{~d}, \mathrm{~J}=8.34 \mathrm{~Hz}, 1 \mathrm{H}), 7.97(\mathrm{~d}$, $\mathrm{J}=8.16 \mathrm{~Hz}, 1 \mathrm{H}), 7.75-7.72(\mathrm{~m}, 1 \mathrm{H}), 7.64-7.61(\mathrm{~m}, 1 \mathrm{H}), 7.35-7.26(\mathrm{~m}, 5 \mathrm{H}), 6.26(\mathrm{dt}, \mathrm{J}=14.94,0.9 \mathrm{~Hz}$, $1 \mathrm{H}), 6.05(\mathrm{dt}, \mathrm{J}=14.82,7.8 \mathrm{~Hz}, 1 \mathrm{H}) 2.50(\mathrm{~s}, 2 \mathrm{H}), 2.31(\mathrm{dd}, \mathrm{J}=7.83,0.9 \mathrm{~Hz}, 2 \mathrm{H}), 1.14(\mathrm{~s}, 6 \mathrm{H}) \mathrm{ppm}$

${ }^{1}$ HNMR cis isomer $\left(\mathbf{6 0 0 M H z}, \mathbf{C D C l}_{3}\right): \delta 9.16(\mathrm{~s}, 1 \mathrm{H}), 8.65(\mathrm{~s}, 1 \mathrm{H}), 8.27(\mathrm{~d}, \mathrm{~J}=8.34 \mathrm{~Hz}, 1 \mathrm{H}), 7.96(\mathrm{~d}, \mathrm{~J}$ $=7.92 \mathrm{~Hz}, 1 \mathrm{H}), 7.74-7.71(\mathrm{~m}, 1 \mathrm{H}), 7.63-7.61(\mathrm{~m}, 1 \mathrm{H}), 7.20-7.17(\mathrm{~m}, 5 \mathrm{H}), 6.38(\mathrm{dt}, \mathrm{J}=9.36,1.02 \mathrm{~Hz}$, $1 \mathrm{H}), 5.95(\mathrm{dt}, \mathrm{J}=9.30,7.68 \mathrm{~Hz}, 1 \mathrm{H}) 2.54(\mathrm{~s}, 2 \mathrm{H}), 2.44(\mathrm{dd}, \mathrm{J}=7.65,1.02 \mathrm{~Hz}, 2 \mathrm{H}), 1.18(\mathrm{~s}, 6 \mathrm{H}) \mathrm{ppm}$

${ }^{13} \mathbf{C N M R}$ of mixture $\boldsymbol{E}$ and $\boldsymbol{Z}\left(\mathbf{6 0 0 M H z}, \mathbf{C D C l}_{3}\right): \delta 151.33,151.25,146.39,136.30,136.20,135.93$, $135.91,132.48,130.94,130.88,129.24,129.01,129.00,128.93,128.70,127.88,127.81,127.72,127.68$, $126.27,125.67,125.28,125.10,124.31,95.98,95.81,77.77,77.74,44.94,40.88,36.69,35.25,34.78$, $33.01,32.71,27.02,26.93,24.72 \mathrm{ppm}$

IR: $v_{\max } 3060,3019,2958,2925,2222,1716,1683,1614,1578,1504,1388,1156,1023,896,761,739$, 689.

HRMS (ESI+) calcd for $\mathrm{C}_{24} \mathrm{H}_{24} \mathrm{NS}^{+}\left[(\mathrm{M}+\mathrm{H})^{+}\right]: 358.16294$, found 358.16259 


\section{Synthesis of malonate-tethered diynyl-pyridine 1 (unoptimized)}

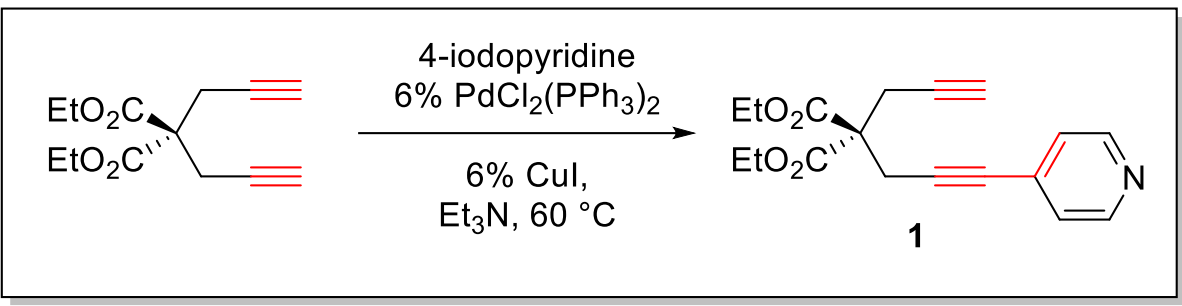

Following the general procedure A, 4-iodopyridine $(0.077 \mathrm{~g}, 0.375 \mathrm{mmol}, 1.00$ equiv) and diethyl 2,2di(prop-2-yn-1-yl)malonate $(0.100 \mathrm{~g}, 0.42 \mathrm{mmol}, 1.13$ equiv. $)$ - prepared according to a previously optimized procedure $^{5}$ - gave $\mathbf{1}(0.042 \mathrm{~g}, 36 \%)$ as a brown oil.

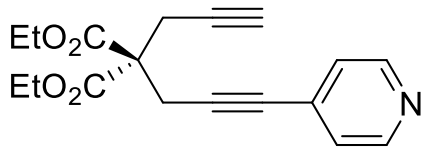

diethyl 2-(prop-2-yn-1-yl)-2-(3-(pyridin-4-yl)prop-2-yn-1-yl)malonate (1)

${ }^{1}$ HNMR (600MHz, $\left.\mathbf{C D C l}_{3}\right): \delta 8.52(\mathrm{~d}, \mathrm{~J}=5.94 \mathrm{~Hz}, 2 \mathrm{H}), 7.21(\mathrm{~d}, \mathrm{~J}=6.00 \mathrm{~Hz}, 2 \mathrm{H}), 4.24(\mathrm{q}, \mathrm{J}=7.08 \mathrm{~Hz}$, 4H), $3.22(\mathrm{~s}, 2 \mathrm{H}), 3.01(\mathrm{~d}, \mathrm{~J}=2.64 \mathrm{~Hz}, 2 \mathrm{H}), 2.05(\mathrm{t}, \mathrm{J}=2.64 \mathrm{~Hz}, 1 \mathrm{H}), 1.26(\mathrm{t}, \mathrm{J}=7.14 \mathrm{~Hz}, 6 \mathrm{H}) \mathrm{ppm}$

${ }^{13}$ CNMR (600MHz, $\left.\mathbf{C D C l}_{3}\right): \delta 168.53,149.69,131.24,125.77,89.36,81.33,78.35,71.89,62.18,56.48$, 23.52, 22.82, $14.06 \mathrm{ppm}$

IR: $v_{\max } 3287,2982,2936,2230,1733,1593,1541,1465,1446,1426,1406,1367,1323,1290,1241$, $1189,1053,1010,856,821$.

HRMS (ESI+) calcd for $\mathrm{C}_{18} \mathrm{H}_{20} \mathrm{NO}_{4}^{+}\left[(\mathrm{M}+\mathrm{H})^{+}\right]: 314.13923$, found 314.13832

\section{Synthesis of Isoquinolines (and Quinolines) via DBU-promoted Benzannulation}
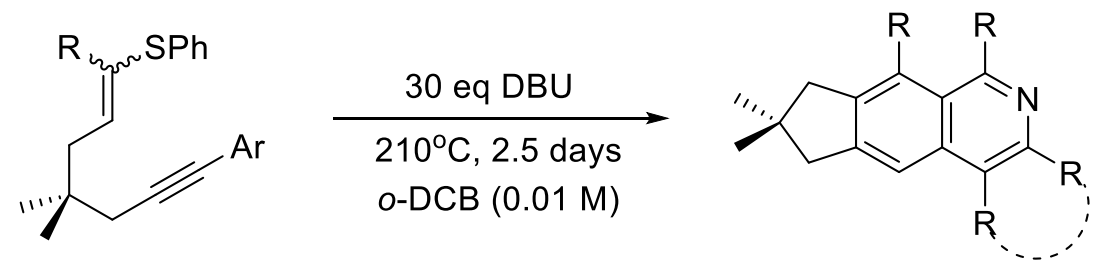

\section{General Procedure B:}

To a 1,2-dichlorobenzene solution $(0.01 \mathrm{M})$ of the appropriate aryl-alkyne (1 equiv.) in a sealed tube was added 1,8-Diazabicyclo[5.4.0] undec-7-ene (30 equiv.). Once sealed, the mixture was heated to $210^{\circ} \mathrm{C}$ for 2.5 days. After 2.5 days the reaction mixture was allowed to cool to room temperature before being

\footnotetext{
${ }^{5}$ Kotha, S.; Ali, R. Tetrahedron 2015, 71, 1597
} 
purified without concentration by flash column chromatography with gradient eluent from EtOAc/Hexane $=1 / 99$ to EtOAc/Hexane $=10 / 90$ to give the desired isoquinolines and quinolines.<smiles>CC1(C)Cc2cc3ccncc3cc2C1</smiles>

\section{7,7-dimethyl-7,8-dihydro-6H-cyclopenta[g]isoquinoline (4)}

Following the general procedure B, aryl-alkyne $3(0.160 \mathrm{~g}, 0.52 \mathrm{mmol})$ afforded $4(0.102 \mathrm{~g}, 81 \%)$ as a yellow solid.

${ }^{1}$ HNMR (600MHz, $\left.\mathbf{C D C l}_{3}\right): \delta 9.14(\mathrm{~s}, 1 \mathrm{H}), 8.41(\mathrm{~d}, \mathrm{~J}=3.72 \mathrm{~Hz}, 1 \mathrm{H}), 7.71(\mathrm{~s}, 1 \mathrm{H}), 7.57(\mathrm{~s}, 1 \mathrm{H}), 7.56(\mathrm{~d}$, $\mathrm{J}=3.72 \mathrm{~Hz}, 1 \mathrm{H}), 2.88(\mathrm{~s}, 2 \mathrm{H}), 2.87(\mathrm{~s}, 2 \mathrm{H}), 1.17(\mathrm{~s}, 6 \mathrm{H}) \mathrm{ppm}$

${ }^{13}$ CNMR (600MHz, $\mathbf{C D C l}_{3}$ ): $\delta$ 151.71, 148.22, 144.64, 142.07, 135.57, 128.44, 122.29, 121.22, 120.30, $47.58,47.26,40.73,28.25 \mathrm{ppm}$

IR: $v_{\max } 3053,2955,2933,2867,1924,1715,1634,1587,1463,1447,1367,1274,1210,907,867,809$.

HRMS (ESI+) calcd for $\mathrm{C}_{14} \mathrm{H}_{16} \mathrm{~N}^{+}\left[(\mathrm{M}+\mathrm{H})^{+}\right]:$198.12827, found 198.12959

MP: $73-77^{\circ} \mathrm{C}$

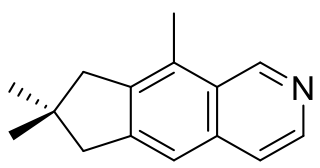

\section{7,7,9-trimethyl-7,8-dihydro-6H-cyclopenta[g]isoquinoline (11)}

Following the general procedure B, aryl-alkyne $\mathbf{1 0}(0.075 \mathrm{~g}, 0.23 \mathrm{mmol})$ afforded $\mathbf{1 1}(0.036 \mathrm{~g}, 73 \%)$ as a white paste.

${ }^{1}$ HNMR (600MHz, $\left.\mathbf{C D C l}_{3}\right): \delta 9.38(\mathrm{~s}, 1 \mathrm{H}), 8.43(\mathrm{~d}, \mathrm{~J}=4.44 \mathrm{~Hz}, 1 \mathrm{H}), 7.55(\mathrm{~d}, \mathrm{~J}=5.52 \mathrm{~Hz}, 1 \mathrm{H}), 7.44(\mathrm{~s}$, $1 \mathrm{H}), 2.88(\mathrm{~s}, 2 \mathrm{H}), 2.85(\mathrm{~s}, 2 \mathrm{H}), 2.64(\mathrm{~s}, 3 \mathrm{H}), 1.18(\mathrm{~s}, 6 \mathrm{H}) \mathrm{ppm}$

${ }^{13}$ CNMR (600MHz, $\left.\mathbf{C D C l}_{3}\right): 148.50,147.63,142.74,141.88,136.03,129.65,120.64,119.28,107.26$, $48.01,46.33,39.88,29.71,28.60 \mathrm{ppm}$

IR: $v_{\max } 2955,2921,2853,1593,1463,1377,1364,1228,870$.

HRMS (ESI+) calcd for $\mathrm{C}_{15} \mathrm{H}_{18} \mathrm{~N}^{+}\left[(\mathrm{M}+\mathrm{H})^{+}\right]: 212.14392$, found 212.14394 


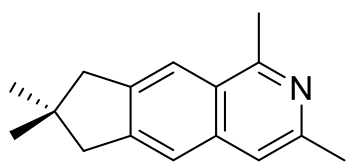

\section{1,3,7,7-tetramethyl-7,8-dihydro-6H-cyclopenta[g]isoquinoline (13)}

Following the general procedure B, aryl-alkyne $12(0.100 \mathrm{~g}, 0.298 \mathrm{mmol})$ afforded $13(0.056 \mathrm{~g}, 84 \%)$ as a beige solid.

${ }^{1}$ HNMR (400MHz, CDCl $)_{3}$ ): $\delta 7.81(\mathrm{~s}, 1 \mathrm{H}), 7.45(\mathrm{~s}, 1 \mathrm{H}), 7.25$ (s, 1H), 2.89 (s, 3H), 2.87 (s, 2H), 2.84 (s, $2 \mathrm{H}), 2.62(\mathrm{~s}, 3 \mathrm{H}), 1.17(\mathrm{~s}, 6 \mathrm{H}) \mathrm{ppm}$

${ }^{13}$ CNMR (600MHz, CDCl 3 ): $\delta$ 157.11, 149.21, 147.42, 143.31, 136.40, 125.13, 121.34, 120.29, 117.08, $47.47,40.67,28.79,28.32,24.16,22.50 \mathrm{ppm}$

IR: $v_{\max } 2957,2927,2867,2833,1980,1637,1599,1567,1465,1429,1366,1297,1174,909,889,867$.

HRMS (ESI+) calcd for $\mathrm{C}_{16} \mathrm{H}_{20} \mathrm{~N}^{+}\left[(\mathrm{M}+\mathrm{H})^{+}\right]: 226.15957$, found 226.16145

MP: $42-43^{\circ} \mathrm{C}$

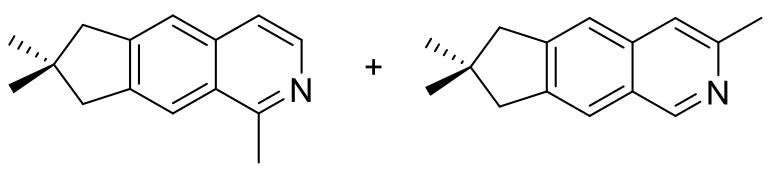

1,7,7-trimethyl-7,8-dihydro-6H-cyclopenta[g]isoquinoline (15a)

3,7,7-trimethyl-7,8-dihydro-6H-cyclopenta[g]isoquinoline (15b)

Following the general procedure B, aryl-alkyne $14(0.025 \mathrm{~g}, 0.08 \mathrm{mmol})$ afforded $15 \mathbf{a}+15 \mathbf{b}(0.015 \mathrm{~g}$, $91 \%$ combined yield) as a 1:0.4 mixture of 15a:15b regioisomers which were not separated.

${ }^{1} \mathbf{H N M R}$ of 15a $\left(\mathbf{6 0 0 M H z}, \mathbf{C D C l}_{3}\right): \delta 8.29(\mathrm{~d}, \mathrm{~J}=5.04 \mathrm{~Hz}, 1 \mathrm{H}), 7.86(\mathrm{~s}, 1 \mathrm{H}), 7.55(\mathrm{~s}, 1 \mathrm{H}), 7.42(\mathrm{~d}, 5.64$ $\mathrm{Hz}, 1 \mathrm{H}), 2.92$ (s, 3H), 2.90 (s, 2H), 2.87 (s, 2H), 1.18 (s, 6H) ppm

${ }^{1}$ HNMR of 15b (600MHz, $\left.\mathbf{C D C l}_{3}\right): \delta 9.05(\mathrm{~s}, 1 \mathrm{H}), 7.65(\mathrm{~s}, 1 \mathrm{H}), 7.47(\mathrm{~s}, 1 \mathrm{H}), 7.38(\mathrm{~s}, 1 \mathrm{H}), 2.85(\mathrm{~s}, 4 \mathrm{H})$, $2.66(\mathrm{~s}, 3 \mathrm{H}), 1.16(\mathrm{~s}, 6 \mathrm{H}) \mathrm{ppm}$

${ }^{13} \mathbf{C N M R}$ of mixture 15a and 15b (600MHz, $\left.\mathbf{C D C l}_{3}\right): \delta 157.64,151.12,150.57,148.10,147.54,144.44$, $143.55,140.83$, 136.40, 135.58, 127.06, 126.62, 122.12, 121.96, 121.76, 120.63, 120.39, 119.17, 118.35, $47.57,47.47,47.18,40.73,40.67,29.71,28.30,28.27,22.70,22.50 \mathrm{ppm}$

HRMS (ESI+) calcd for $\mathrm{C}_{15} \mathrm{H}_{18} \mathrm{~N}^{+}\left[(\mathrm{M}+\mathrm{H})^{+}\right]: 212.14392$, found 212.14376<smiles>CC1(C)Cc2cc3cccnc3cc2C1</smiles> 


\section{7,7-dimethyl-7,8-dihydro-6H-cyclopenta[g]quinolone (17b)}

Following the general procedure B, aryl-alkyne $16(0.020 \mathrm{~g}, 0.065 \mathrm{mmol})$ afforded $17 \mathbf{a}+\mathbf{1 7 b}(0.0094 \mathrm{~g}$, $73 \%$ ) as a $\mathbf{1 : 1}$ mixture of $\mathbf{1 7 a : 1 7 b}$ regioisomers which were not separated.

${ }^{1}$ HNMR of 17a (400MHz, $\mathrm{CDCl}_{3}$ ): Refer to compound 4 (above).

${ }^{1}$ HNMR of 17b (400MHz, $\left.\mathbf{C D C l}_{3}\right): \delta 8.83(\mathrm{~d}, \mathrm{~J}=4.08 \mathrm{~Hz}, 1 \mathrm{H}), 8.08(\mathrm{~d}, \mathrm{~J}=8.2 \mathrm{~Hz}, 1 \mathrm{H}), 7.87(\mathrm{~s}, 1 \mathrm{H})$, $7.58(\mathrm{~s}, 1 \mathrm{H}), 7.34-7.31(\mathrm{~m}, 1 \mathrm{H}), 2.94(\mathrm{~s}, 2 \mathrm{H}), 2.90(\mathrm{~s}, 2 \mathrm{H}), 1.20(\mathrm{~s}, 6 \mathrm{H}) \mathrm{ppm}$

${ }^{13} \mathbf{C N M R}$ of mixture 17a and 17b (600MHz, $\left.\mathbf{C D C l}_{3}\right): \delta 151.62,149.03,148.30,148.08,147.42,144.71$, $143.89,141.94,135.60,131.69,127.59,126.87,124.07,122.32,122.32,122.22,121.24,120.61,120.00$, $47.60,47.27,47.26,47.20,40.89,40.74,29.71,28.31,28.25 \mathrm{ppm}$

HRMS (ESI+) calcd for $\mathrm{C}_{14} \mathrm{H}_{16} \mathrm{~N}^{+}\left[(\mathrm{M}+\mathrm{H})^{+}\right]: 198.12827$, found 198.12852<smiles>CC1(C)Cc2cc3cncc(Cl)c3cc2C1</smiles>

\section{4-chloro-7,7-dimethyl-7,8-dihydro-6H-cyclopenta[g]isoquinoline (19)}

Following the general procedure B, aryl-alkyne $18(0.160 \mathrm{~g}, 0.47 \mathrm{mmol})$ afforded $\mathbf{1 9}(0.076 \mathrm{~g}, 71 \%)$ as a beige solid.

${ }^{1}$ HNMR (400MHz, CDCl $\mathbf{3}_{3}$ ): $\delta 9.01$ (s, 1H), 8.47 (s, 1H), 7.95 (s, 1H), 7.73 (s, 1H), 2.94 (s, 2H), 2.91 (s, 2H), $1.19(\mathrm{~s}, 6 \mathrm{H}) \mathrm{ppm}$

${ }^{13}$ CNMR (600MHz, $\left.\mathbf{C D C l}_{3}\right): \delta 150.37,149.64,145.64,141.07,133.17,129.24,128.13,122.41,118.11$, $47.78,47.18,40.83,28.21 \mathrm{ppm}$

IR: $v_{\max } 3015,2951,2937,2861,1730,1637,1577,1466,1232,1094,912,902,859$.

HRMS (ESI+) calcd for $\mathrm{C}_{14} \mathrm{H}_{15} \mathrm{ClN}^{+}\left[(\mathrm{M}+\mathrm{H})^{+}\right]$: 232.08930, found 232.09180

MP: $57-62^{\circ} \mathrm{C}$<smiles>CC1(C)Cc2cc3cnc4ccccc4c3cc2C1</smiles>

\section{9,9-dimethyl-9,10-dihydro-8H-cyclopenta[j]phenanthridine (21)}

Following the general procedure B, aryl-alkyne $20(0.075 \mathrm{~g}, 0.21 \mathrm{mmol})$ afforded $21(0.044 \mathrm{~g}, 86 \%)$ as a yellow solid. 
${ }^{1}$ HNMR (400MHz, CDCl3): $\delta 9.20(\mathrm{~s}, 1 \mathrm{H}), 8.54(\mathrm{~d}, \mathrm{~J}=7.40 \mathrm{~Hz}, 1 \mathrm{H}), 8.39$ (s, 1H), 8.16 (d, J = $7.92 \mathrm{~Hz}$, 1H), $7.80(\mathrm{~s}, 1 \mathrm{H}), 7.70(\mathrm{td}, \mathrm{J}=7.48,1.20 \mathrm{~Hz}, 1 \mathrm{H}), 7.64(\mathrm{td}, \mathrm{J}=7.56,1.28 \mathrm{~Hz}, 1 \mathrm{H}), 2.99(\mathrm{~s}, 2 \mathrm{H}), 2.94(\mathrm{~s}$, 2H), $1.22(\mathrm{~s}, 6 \mathrm{H}) \mathrm{ppm}$

${ }^{13}$ CNMR (600MHz, $\mathbf{C D C l}_{3}$ ): $\delta$ 153.42, 148.61, 144.36, 144.30, 131.82, 129.97, 128.04, 126.67, 125.84, $124.52,124.03,122.10,117.26,48.10,47.32,40.89,28.43 \mathrm{ppm}$

IR: $v_{\max } 3071,2947,2930,2862,1630,1588,1574,1456,1373,1363,1181,1096,949,939,863,753$.

HRMS (ESI+) calcd for $\mathrm{C}_{18} \mathrm{H}_{18} \mathrm{~N}^{+}\left[(\mathrm{M}+\mathrm{H})^{+}\right]: 248.14392$, found 248.14452

MP: $100-104^{\circ} \mathrm{C}$<smiles></smiles>

\section{9,9-dimethyl-9,10-dihydro-8H-cyclopenta[b]phenanthridine (23)}

Following the general procedure B, aryl-alkyne $22(0.040 \mathrm{~g}, 0.11 \mathrm{mmol})$ afforded $23(0.027 \mathrm{~g},>95 \%)$ as a yellow solid.

${ }^{1}$ HNMR (600MHz, $\left.\mathbf{C D C l}_{3}\right): \delta 9.21(\mathrm{~s}, 1 \mathrm{H}), 8.57(\mathrm{~d}, \mathrm{~J}=8.28 \mathrm{~Hz}, 1 \mathrm{H}), 8.36(\mathrm{~s}, 1 \mathrm{H}), 8.02(\mathrm{~d}, \mathrm{~J}=7.74 \mathrm{~Hz}$, 1H), 7.95 (s, 1H), 7.82 (td, J = 7.65, 1.26 Hz, 1H), 7.66 (td, J = 7.47, $0.66 \mathrm{~Hz}, 1 \mathrm{H}), 2.97$ (s, 2H), 2.96 (s, $2 \mathrm{H}), 1.22(\mathrm{~s}, 6 \mathrm{H}) \mathrm{ppm}$

${ }^{13}$ CNMR (600MHz, $\left.\mathbf{C D C l}_{3}\right): \delta$ 152.22, 145.83, 144.09, 143.91, 132.82, 130.66, 128.67, 126.80, 126.08, $125.30,122.79,121.74,117.32,47.76,47.61,41.02,29.71,28.51 \mathrm{ppm}$

IR: $v_{\max } 3068,2951,2922,2854,1769,1715,1616,1585,1574,1479,1455,1444,1364,1251,1097$, $1028,863,793,748$.

HRMS (ESI+) calcd for $\mathrm{C}_{18} \mathrm{H}_{18} \mathrm{~N}^{+}\left[(\mathrm{M}+\mathrm{H})^{+}\right]: 248.14392$, found 248.14447

MP: $76-82{ }^{\circ} \mathrm{C}$

\section{Synthesis of Brominated Isoquinolines}

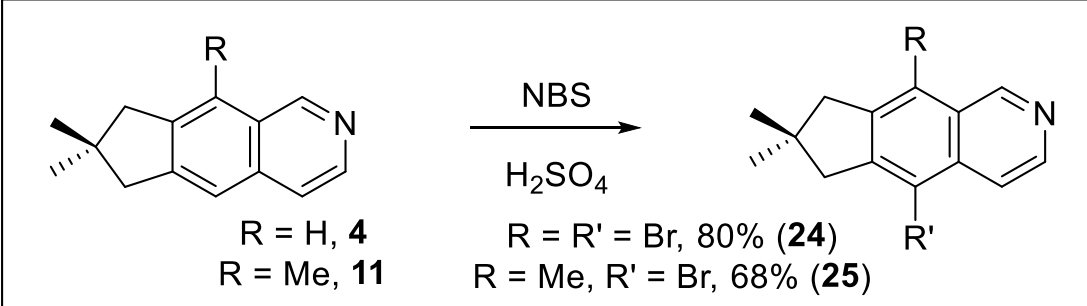


<smiles>CC1(C)Cc2c(c(Br)c3cnccc3c2Br)C1</smiles>

\section{5,9-dibromo-7,7-dimethyl-7,8-dihydro-6H-cyclopenta[g]isoquinoline (24)}

Compound 24 was prepared according to a procedure adapted from Gouliaev. ${ }^{6}$ To stirred, concentrated $\mathrm{H}_{2} \mathrm{SO}_{4}(0.3 \mathrm{~mL})$ was added isoquinoline $4(0.027 \mathrm{~g}, 0.136 \mathrm{mmol}, 1$ equiv.). The mixture was cooled to 0 ${ }^{\circ} \mathrm{C}$ and N-bromosuccinimide ( $0.053 \mathrm{~g}, 0.2992 \mathrm{mmol}, 2.2$ equiv.) was slowly added. After 5 minutes at 0 ${ }^{\circ} \mathrm{C}$, the mixture was allowed to warm to room temperature for 18 hours before being diluted with $\mathrm{H}_{2} \mathrm{O}$. The $\mathrm{pH}$ was adjusted to 9 with $\mathrm{NH}_{4} \mathrm{OH}$ and the reaction mixture was extracted with methylene chloride 4 times. The combined organic layers were washed with one portion of $1 \mathrm{M} \mathrm{NaOH}$, saturated $\mathrm{NaCl}$, and dried over $\mathrm{MgSO}_{4}$ before being concentrated in vacuo. The crude residue was passed through a silica plug with eluent EtOAc/Hexanes = 40/60 to give pure $24(0.038 \mathrm{~g}, 80 \%)$ as an off-white solid.

${ }^{1}$ HNMR (600MHz, CDCl $)$ ): $\delta 9.53(\mathrm{~s}, 1 \mathrm{H}), 8.64(\mathrm{~d}, \mathrm{~J}=5.7,1 \mathrm{H}), 7.93(\mathrm{~d}, \mathrm{~J}=5.82,1 \mathrm{H}), 3.07(\mathrm{~s}, 4 \mathrm{H})$, $1.24(\mathrm{~s}, 6 \mathrm{H}) \mathrm{ppm}$

${ }^{13}$ CNMR (600MHz, $\left.\mathbf{C D C l}_{3}\right): \delta 151.16,148.52,145.40,144.51,135.98,118.63,118.18,117.22,50.83$, $50.29,38.81,28.68 \mathrm{ppm}$

IR: $v_{\max } 3061,2949,2919,2853,1916,1621,1585,1460,1415,1252,1093,1050,937,918,815$.

HRMS (ESI+) calcd for $\mathrm{C}_{14} \mathrm{H}_{14} \mathrm{Br}_{2} \mathrm{~N}^{+}\left[(\mathrm{M}+\mathrm{H})^{+}\right]: 353.94930$, found 353.94871

MP: $156-160^{\circ} \mathrm{C}$<smiles>Cc1c2c(c(Br)c3ccncc13)CC(C)(C)C2</smiles>

5-bromo-7,7,9-trimethyl-7,8-dihydro-6H-cyclopenta[g]isoquinoline (25)

Following the same procedure as $\mathbf{2 4}$, to stirred, concentrated $\mathrm{H}_{2} \mathrm{SO}_{4}(0.25 \mathrm{~mL})$ was added isoquinoline 4 (0.016 g, $0.075 \mathrm{mmol}, 1$ equiv.). The mixture was cooled to $0{ }^{\circ} \mathrm{C}$ and $\mathrm{N}$-bromosuccinimide $(0.015 \mathrm{~g}$, $0.082 \mathrm{mmol}, 1.1$ equiv.) was slowly added. After 5 minutes at $0{ }^{\circ} \mathrm{C}$, the mixture was allowed to warm to room temperature for 18 hours before being diluted with $\mathrm{H}_{2} \mathrm{O}$. The $\mathrm{pH}$ was adjusted to 9 with $\mathrm{NH}_{4} \mathrm{OH}$ and the reaction mixture was extracted with methylene chloride 4 times. The combined organic layers were washed with one portion of $1 \mathrm{M} \mathrm{NaOH}$, saturated $\mathrm{NaCl}$, and dried over $\mathrm{MgSO}_{4}$ before being concentrated in vacuo. The crude residue was passed through a silica plug with eluent EtOAc/Hexanes $=40 / 60$ to give pure $25(0.015 \mathrm{~g}, 68 \%)$ as a light-brown solid.

\footnotetext{
${ }^{6}$ Brown, W. D.; Gouliaev, A. H. Synthesis 2002, 01, 0083
} 
${ }^{1}$ HNMR (600MHz, $\left.\mathbf{C D C l}_{3}\right): \delta 9.36(\mathrm{~s}, 1 \mathrm{H}), 8.57(\mathrm{~d}, \mathrm{~J}=5.64,1 \mathrm{H}), 7.96(\mathrm{~d}, \mathrm{~J}=5.82), 2.99(\mathrm{~s}, 1 \mathrm{H}), 2.98$ (s, 1H), $2.62(\mathrm{~s}, 3 \mathrm{H}), 1.22(\mathrm{~s}, 1 \mathrm{H}) \mathrm{ppm}$

${ }^{13}$ CNMR (600MHz, $\left.\mathbf{C D C l}_{3}\right): \delta 148.44,148.31,143.15,142.91,134.88,129.38,128.38,119.25,115.39$, $50.22,47.50,38.95,29.71,28.80,14.52 \mathrm{ppm}$

IR: $v_{\max } 3059,2948,2922,2862,1912,1717,1622,1589,1465,1420,1373,1272,1178,1028,915,817$.

HRMS (ESI+) calcd for $\mathrm{C}_{15} \mathrm{H}_{17} \mathrm{BrN}^{+}\left[(\mathrm{M}+\mathrm{H})^{+}\right]: 290.05444$, found 290.05431

MP: $125-129^{\circ} \mathrm{C}$

\section{Site-selective substitution of 13}

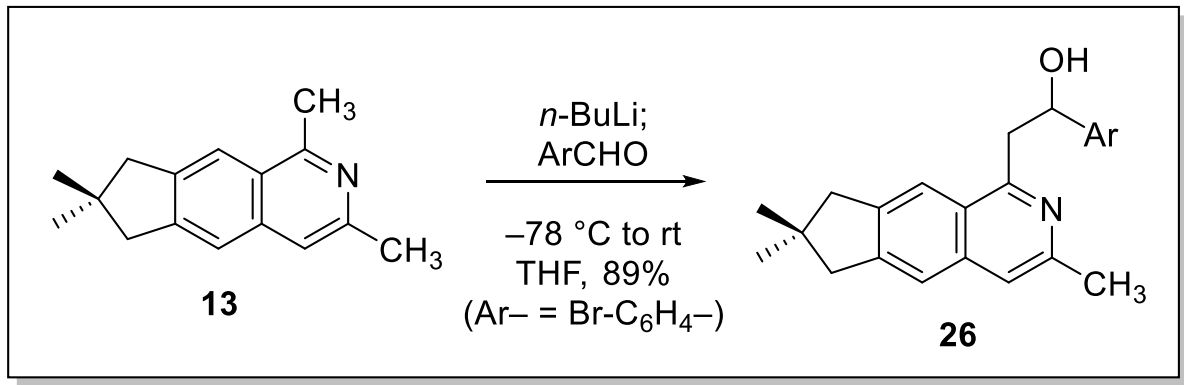

1-(4-bromophenyl)-2-(3,7,7-trimethyl-7,8-dihydro-6H-cyclopenta[g]isoquinolin-1-yl)ethan-1-ol (26)

To $0.32 \mathrm{~mL}$ THF solution of isoquinoline $13(0.0145 \mathrm{~g}, 0.064 \mathrm{mmol}, 1$ equiv. $)$ at $-78{ }^{\circ} \mathrm{C}$ was slowly added $0.04 \mathrm{~mL}$ of $n$-BuLi (1.6 M solution in hexanes, 1 equiv.). The reaction mixture was stirred at $-78{ }^{\circ} \mathrm{C}$ for 30 minutes before 4-bromobenzaldehyde ( $0.036 \mathrm{~g}, 0.193 \mathrm{mmol}, 3$ equiv.) in $0.1 \mathrm{~mL}$ of THF was added dropwise via syringe. The reaction was immediately warmed to $0{ }^{\circ} \mathrm{C}$ for 5 minutes before being brought to room temperature for 1.5 hours. After 1.5 hours, the reaction mixture was quenched with $\mathrm{H}_{2} \mathrm{O}$, extracted with diethyl ether 3 times, and the combined organic layers were washed with saturated $\mathrm{NaCl}$, dried over $\mathrm{MgSO}_{4}$, and concentrated in vacuo. The crude residue was purified by flash column chromatography with gradient eluent from EtOAc/Hexane $=10 / 90$ to EtOAc/Hexane $=20 / 80$ to give 26 $(0.0235 \mathrm{~g}, 89 \%)$ as a yellow solid.

${ }^{1}$ HNMR (600MHz, $\left.\mathbf{C D C l}_{3}\right): \delta 7.69(\mathrm{~s}, 1 \mathrm{H}), 7.50(\mathrm{~d}, \mathrm{~J}=8.4 \mathrm{~Hz}, 2 \mathrm{H}), 7.48(\mathrm{~s}, 1 \mathrm{H}), 7.40$ (d, J= $8.34 \mathrm{~Hz}$, 2H), $7.32(\mathrm{~s}, 1 \mathrm{H}), 5.34(\mathrm{dd}, \mathrm{J}=9.9,2.16 \mathrm{~Hz}, 1 \mathrm{H}) 3.62(\mathrm{~d}, \mathrm{~J}=16.32,1 \mathrm{H}), 3.32(\mathrm{dd}, \mathrm{J}=12,10.02 \mathrm{~Hz}, 1 \mathrm{H})$, $2.85(\mathrm{~s}, 2 \mathrm{H}), 2.82(\mathrm{~s}, 2 \mathrm{H}), 2.65(\mathrm{~s}, 3 \mathrm{H}), 1.16(\mathrm{~s}, 6 \mathrm{H}) \mathrm{ppm}$

${ }^{13}$ CNMR (600MHz, $\left.\mathbf{C D C l}_{3}\right): \delta 158.07,144.06,143.49,136.82,131.64,131.42,128.58,127.78,124.81$, $121.66,120.93,119.26,117.82 \mathrm{ppm}$

IR: $v_{\max } 3488,3254,2952,2922,2852,1717,1640,1602,1564,1485,1461,1425,1067,1009,908,881$, 820.

HRMS (ESI+) calcd for $\mathrm{C}_{23} \mathrm{H}_{25} \mathrm{BrNO}^{+}\left[(\mathrm{M}+\mathrm{H})^{+}\right]: 410.11195$, found 410.11127 
MP: $93-96^{\circ} \mathrm{C}$

\section{Synthesis of Pentacyclic Phenanthridine 28}
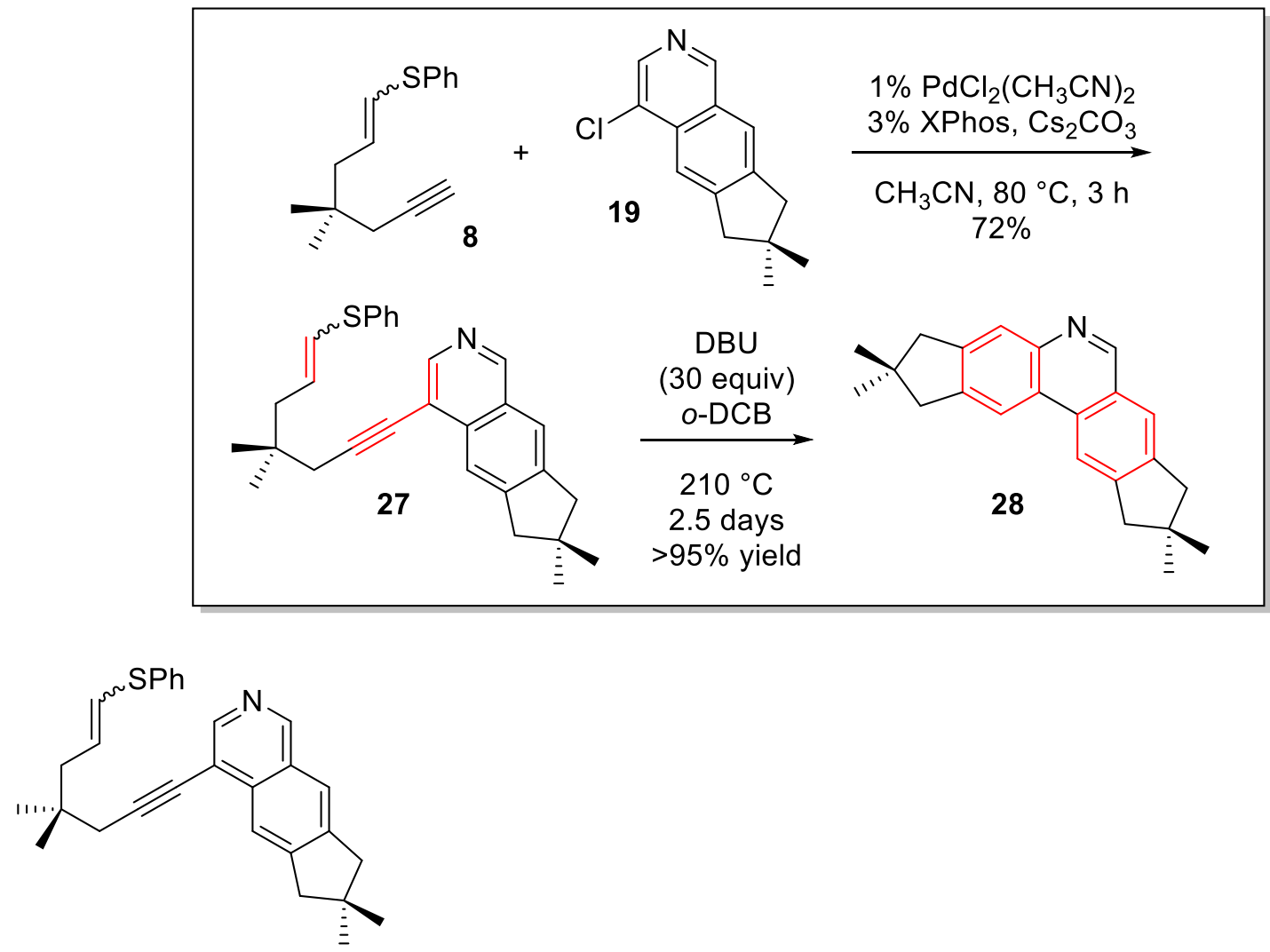

\section{4-(4,4-dimethyl-7-(phenylthio)hept-6-en-1-yn-1-yl)-7,7-dimethyl-7,8-dihydro-6H-} cyclopenta[g]isoquinoline (27)

Compound 27 was prepared according to a procedure adapted from Buchwald. ${ }^{8}$ An oven-dried flask under the flow of $\mathrm{N}_{2}$ was charged with $\mathrm{PdCl}_{2}\left(\mathrm{CH}_{3} \mathrm{CN}\right)_{2}(0.006 \mathrm{~g}, 0.0023 \mathrm{mmol}, 0.01$ equiv.), XPhos (0.0033 g, 0.0069 mmol, 0.03 equiv.), and $\mathrm{Cs}_{2} \mathrm{CO}_{3}(0.15 \mathrm{~g}, 0.46 \mathrm{mmol}, 2$ equiv.). The flask was further vacuumed and filled with $\mathrm{N}_{2} 3$ times before the addition of $0.5 \mathrm{~mL}$ of degassed $\mathrm{CH}_{3} \mathrm{CN}(0.5 \mathrm{M})$ and aryl chloride 19 ( $0.054 \mathrm{~g}, 0.23 \mathrm{mmol}, 1$ equiv.). The slightly yellow suspension was stirred for 25 minutes before slow, dropwise addition of enyne $8\left(0.069 \mathrm{~g}, 0.30 \mathrm{mmol}, 1.3\right.$ equiv.) and heating to $80^{\circ} \mathrm{C}$ for 3 hours. After 3 hours the reaction mixture was allowed to cool to room temperature, diluted with $15 \mathrm{~mL}$ of $\mathrm{H}_{2} \mathrm{O}$, and extracted with diethyl ether $(4 \times 20 \mathrm{~mL})$. The combined organic layers were dried over $\mathrm{MgSO}_{4}$, concentrated, and the residue was purified by flash column chromatography (eluent mixture: EtOAc/Hexane $=10 / 90)$ to give $27(0.071 \mathrm{~g}, 72 \%, E: Z=3.3: 1)$ as a yellow oil.

\footnotetext{
${ }^{8}$ Gelman, D.; Buchwald, S. L. Angew. Chem., Int. Ed. 2003, 42, 5993
} 
${ }^{1}$ HNMR trans isomer $\left(\mathbf{6 0 0 M H z}, \mathbf{C D C l}_{3}\right): \delta 9.02(\mathrm{~s}, 1 \mathrm{H}), 8.54(\mathrm{~s}, 1 \mathrm{H}), 7.97(\mathrm{~s}, 1 \mathrm{H}), 7.70(\mathrm{~s}, 1 \mathrm{H}), 7.35$ $7.27(\mathrm{~m}, 5 \mathrm{H}), 6.27(\mathrm{~d}, \mathrm{~J}=14.88 \mathrm{~Hz}, 1 \mathrm{H}), 6.06(\mathrm{dt}, \mathrm{J}=14.82,7.8 \mathrm{~Hz}, 1 \mathrm{H}) 2.88(\mathrm{~s}, 2 \mathrm{H}) 2.87$ (s, 2H), 2.49 (s, 2H), $2.32(\mathrm{~d}, \mathrm{~J}=7.8 \mathrm{~Hz}, 2 \mathrm{H}), 1.16(\mathrm{~s}, 6 \mathrm{H}), 1.14(\mathrm{~s}, 6 \mathrm{H}) \mathrm{ppm}$

${ }^{1}$ HNMR cis isomer $\left(\mathbf{6 0 0 M H z}, \mathbf{C D C l}_{3}\right): \delta 9.02(\mathrm{~s}, 1 \mathrm{H}), 8.54(\mathrm{~s}, 1 \mathrm{H}), 8.02(\mathrm{~s}, 1 \mathrm{H}), 7.70(\mathrm{~s}, 1 \mathrm{H}), 7.23-7.16$ $(\mathrm{m}, 5 \mathrm{H}), 6.37(\mathrm{~d}, \mathrm{~J}=9.36,7.68 \mathrm{~Hz}, 1 \mathrm{H}), 5.95(\mathrm{dt}, \mathrm{J}=9.3,7.68 \mathrm{~Hz}, 1 \mathrm{H}) 2.88(\mathrm{~s}, 2 \mathrm{H}), 2.87(\mathrm{~s}, 2 \mathrm{H}), 2.54(\mathrm{~s}$, $2 \mathrm{H}), 2.44(\mathrm{~d}, \mathrm{~J}=7.62,2 \mathrm{H}), 1.17(\mathrm{~s}, 6 \mathrm{H}) \mathrm{ppm}$

${ }^{13} \mathbf{C N M R}$ of mixture $\boldsymbol{E}$ and $\boldsymbol{Z}\left(\mathbf{6 0 0 M H z}, \mathbf{C D C l}_{3}\right): \delta 150.53,149.08,149.02,145.60,145.07,145.02$, $136.32,136.26,135.68,135.66,132.77,129.37,129.26,129.15,128.99,128.98,128.61,127.53,126.28$, 126.22 , 125.64, 124.14, 122.54, 122.48, 120.03, 119.88, 119.47, 116.18, 95.19, 95.03, 78.26, 78.21, $47.78,47.74,47.21,44.90,40.89,40.76,36.67,35.25,34.78,33.08,32.71,29.71,28.25,27.02,26.91$ ppm

IR: $v_{\max } 3067,3020,2953,2925,2866,2234,1717,1683,1575,1463,1365,1157,907,894,788,689$.

HRMS (ESI+) calcd for $\mathrm{C}_{29} \mathrm{H}_{32} \mathrm{NS}^{+}\left[(\mathrm{M}+\mathrm{H})^{+}\right]$: 426.22554 , found 426.22566

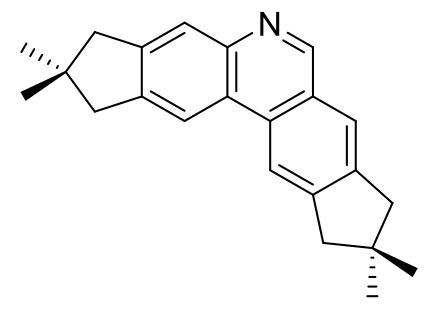

\section{2,2,9,9-tetramethyl-1,2,3,8,9,10-hexahydrodicyclopenta[b,j]phenanthridine (28)}

Following the general procedure B, aryl-alkyne $27(0.060 \mathrm{~g}, 0.14 \mathrm{mmol})$ afforded $28(0.044 \mathrm{~g},>95 \%)$ as a white solid.

${ }^{1}$ HNMR (600MHz, CDCl $)$ ): $\delta 9.11(\mathrm{~s}, 1 \mathrm{H}), 8.34(\mathrm{~s}, 1 \mathrm{H}), 8.31(\mathrm{~s}, 1 \mathrm{H}), 7.91(\mathrm{~s}, 1 \mathrm{H}), 7.75(\mathrm{~s}, 1 \mathrm{H}), 2.95(\mathrm{~s}$, 2H), $2.94(\mathrm{~s}, 4 \mathrm{H}), 2.91(\mathrm{~s}, 2 \mathrm{H}), 1.21(\mathrm{~s}, 6 \mathrm{H}), 1.20(\mathrm{~s}, 6 \mathrm{H}) \mathrm{ppm}$

${ }^{13}$ CNMR (600MHz, $\left.\mathbf{C D C l}_{3}\right): \delta 152.10,148.10,145.03,143.82,143.54,132.03,128.64,125.51,125.15$, $123.79,123.16,117.17,117.06,48.07,47.76,47.59,47.28,40.96,40.83,28.53,28.44$ ppm

IR: $v_{\max } 2953,2921,2862,1629,1589,1575,1502,1451,1364,1246,1166,1098,931,855$.

HRMS (ESI+) calcd for $\mathrm{C}_{23} \mathrm{H}_{26} \mathrm{~N}^{+}\left[(\mathrm{M}+\mathrm{H})^{+}\right]: 316.20652$, found 316.20826

MP: $145-147^{\circ} \mathrm{C}$ 


\section{Deuterium Labeling Experiments}
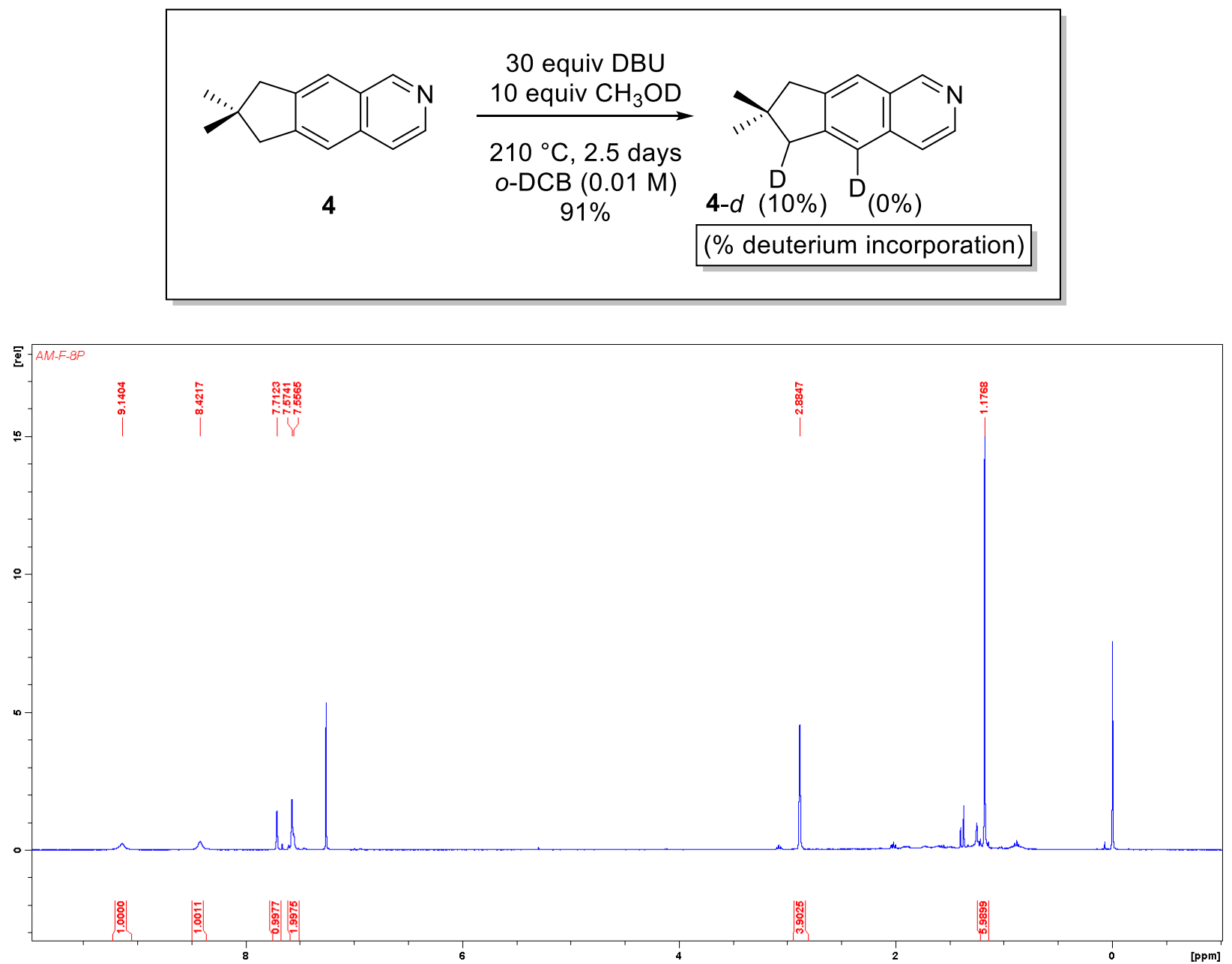

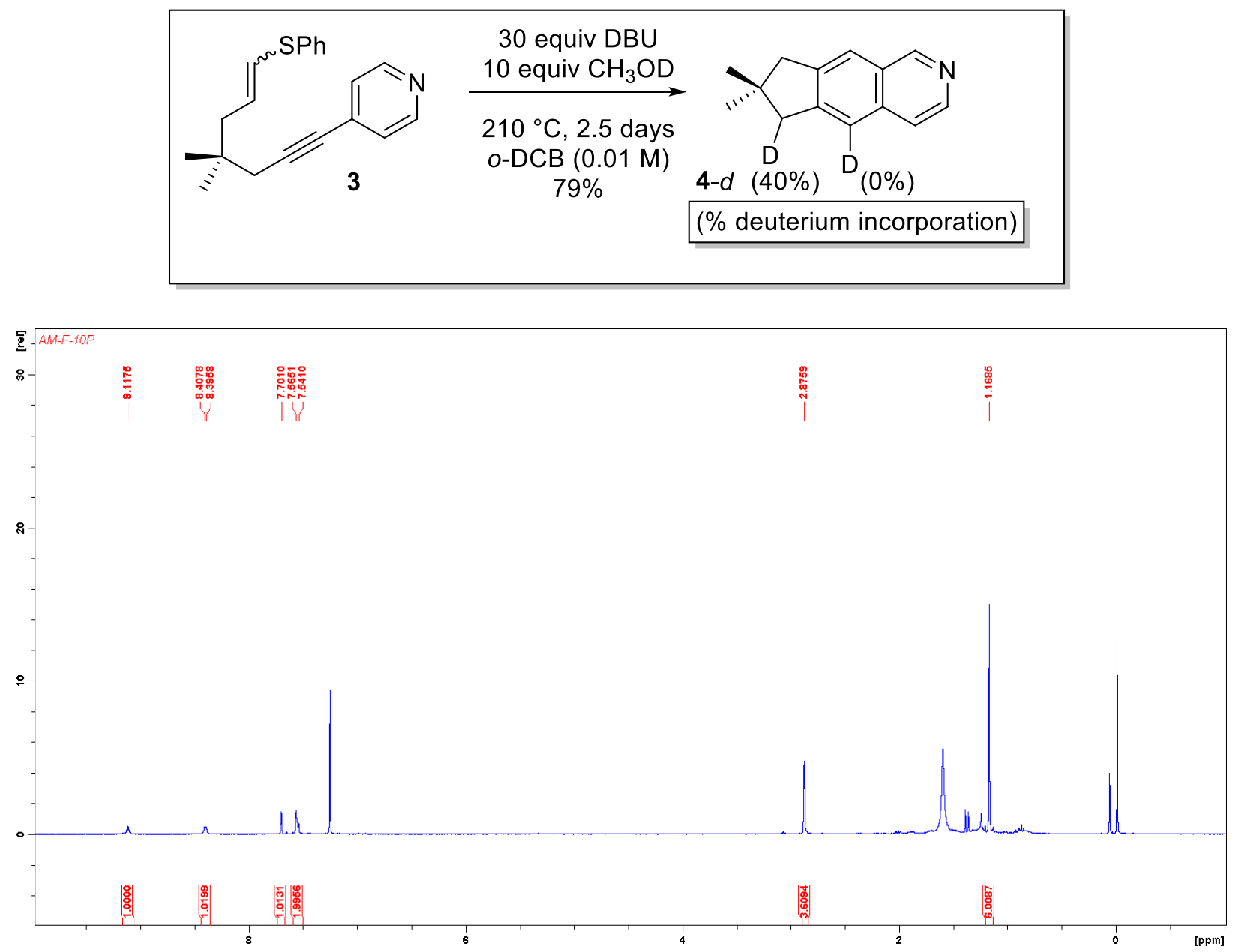


\section{Copy of ${ }^{1} \mathrm{H}-\mathrm{NMR}$ and ${ }^{13} \mathrm{C}-\mathrm{NMR}$ Data}<smiles>C#CCC(C)(C)C/C=C/[SbH]c1ccccc1</smiles>

(4,4-dimethylhept-1-en-6-yn-1-yl)(phenyl)sulfane (8)
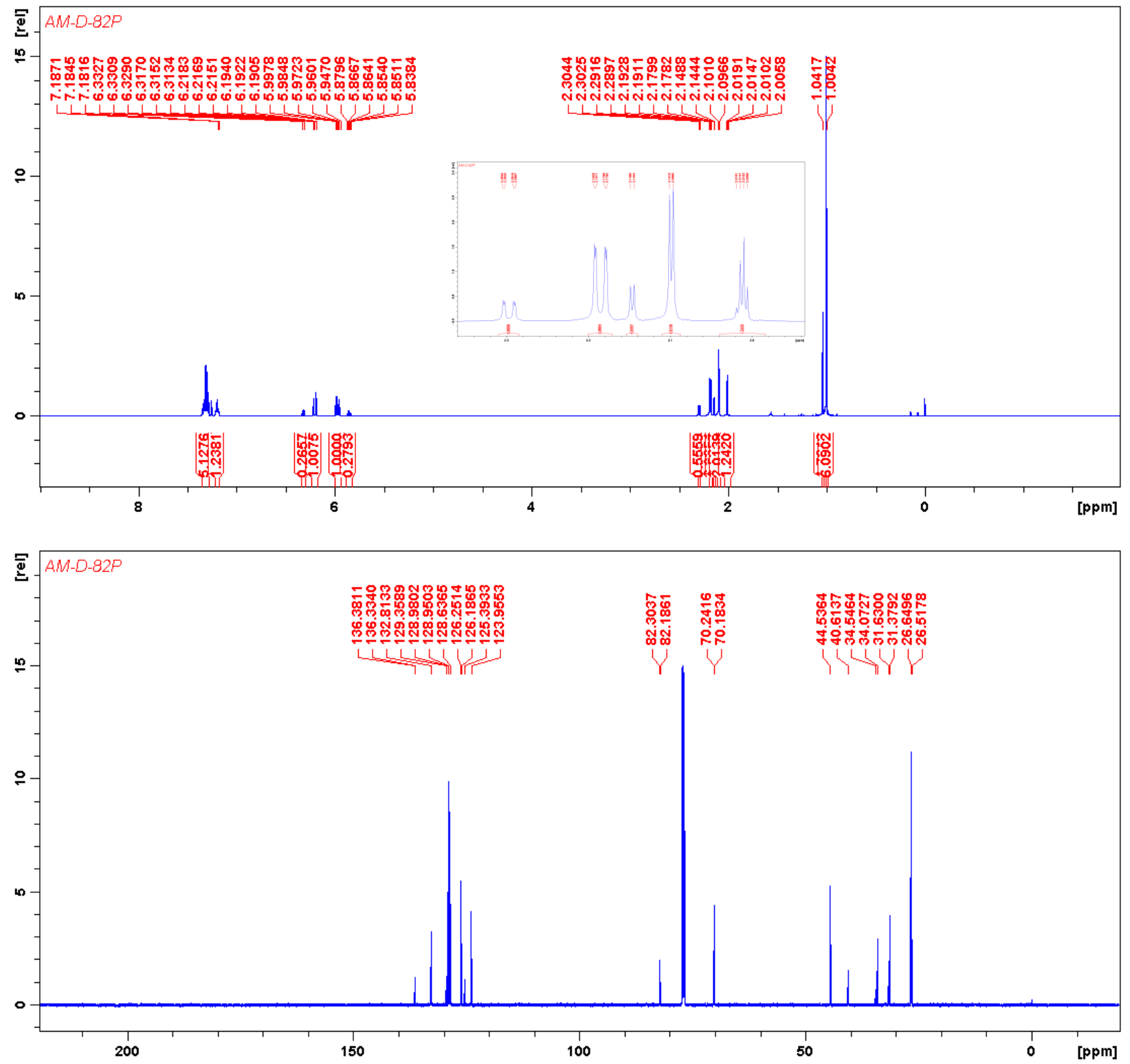


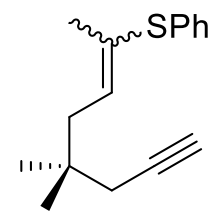

(5,5-dimethyloct-2-en-7-yn-2-yl)(phenyl)sulfane (9)
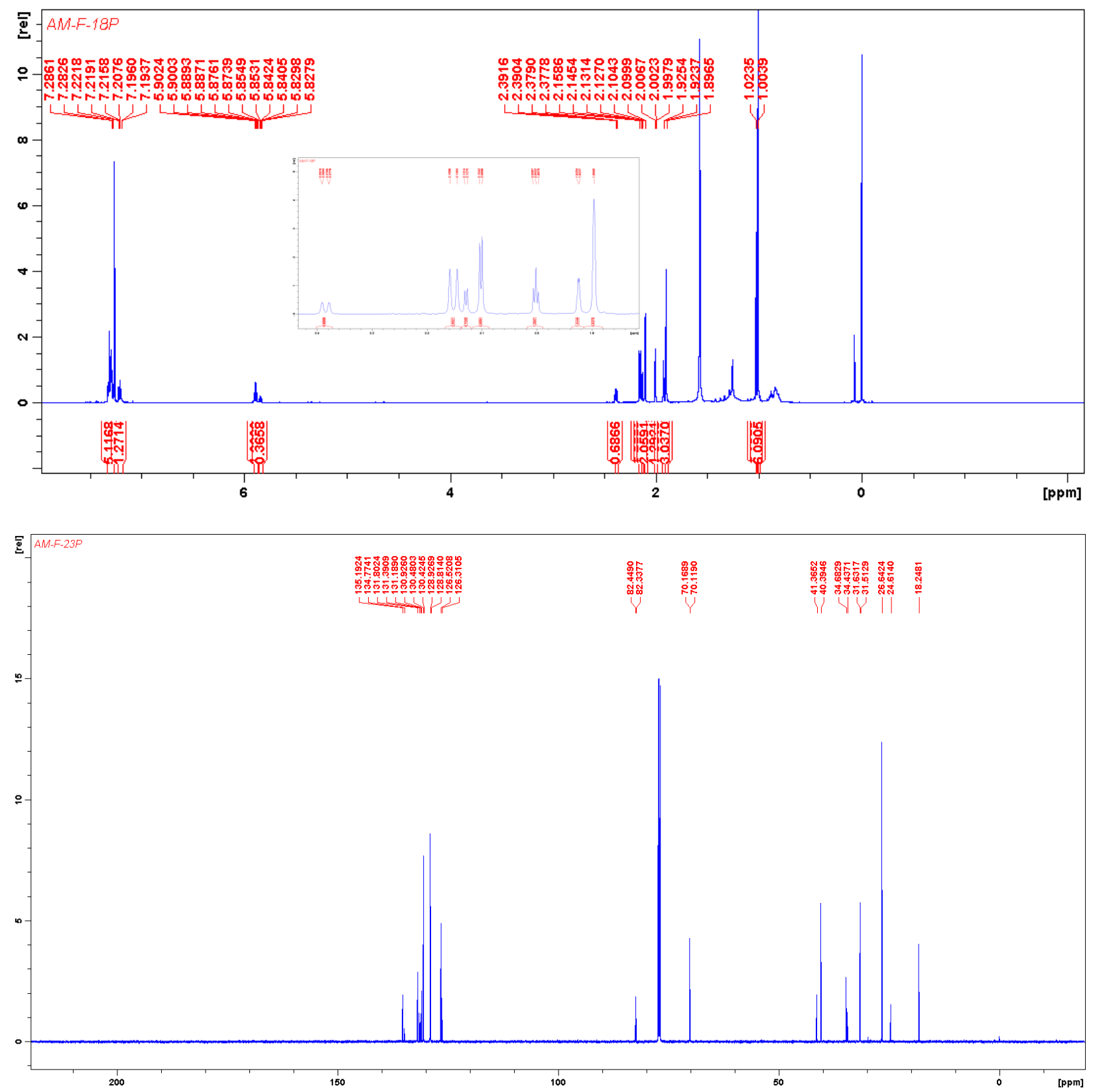


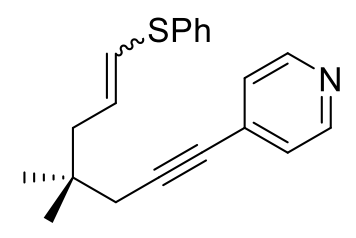

\section{4-(4,4-dimethyl-7-(phenylthio)hept-6-en-1-yn-1-yl)pyridine (3)}
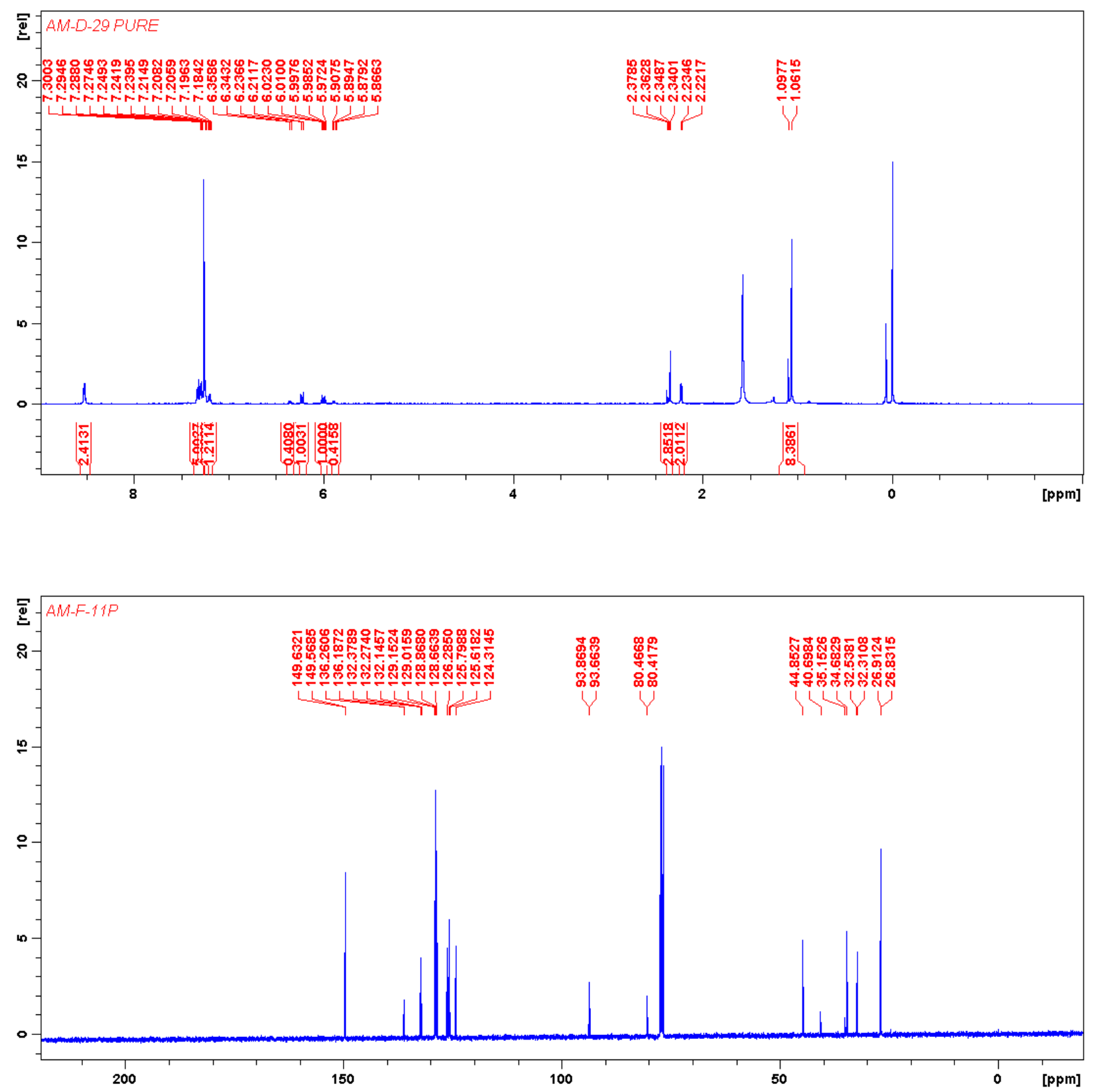


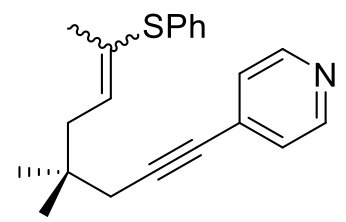

4-(4,4-dimethyl-7-(phenylthio)oct-6-en-1-yn-1-yl)pyridine (10)
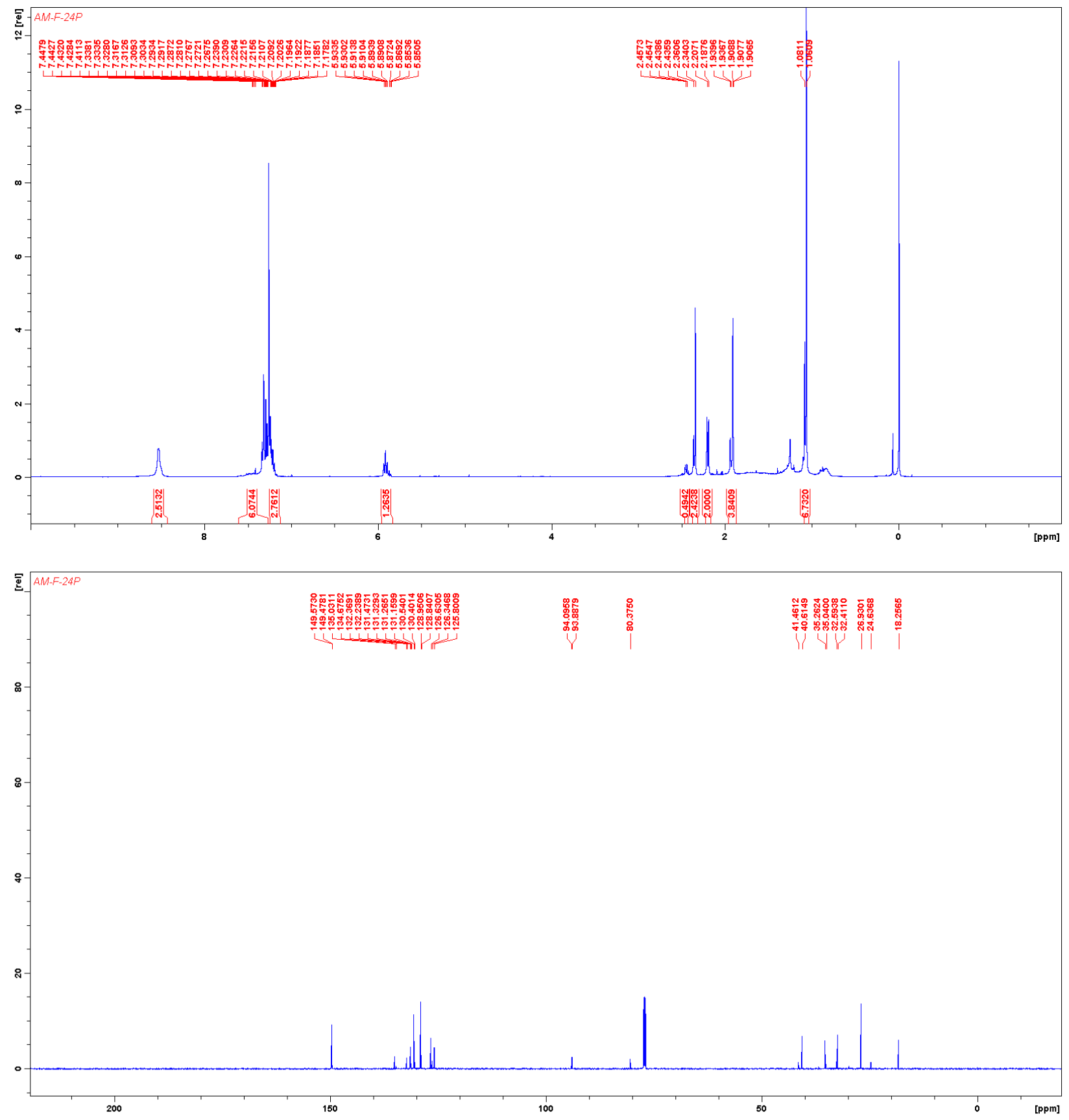
<smiles>Cc1cc(C#CCC(C)(C)C/C=C/[SbH]c2ccccc2)cc(C)n1</smiles>

4-(4,4-dimethyl-7-(phenylthio)hept-6-en-1-yn-1-yl)-2,6-dimethylpyridine (12)
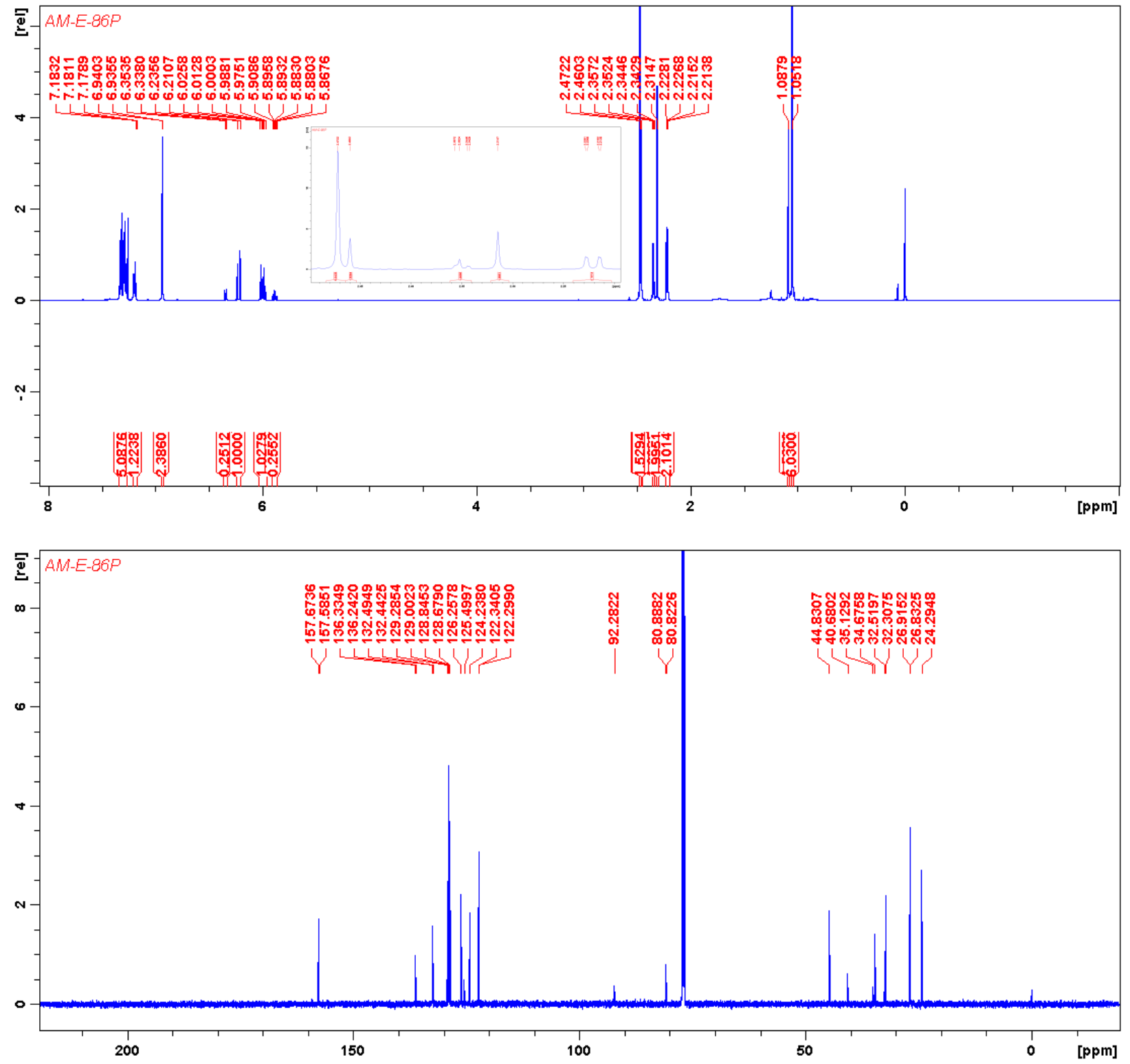


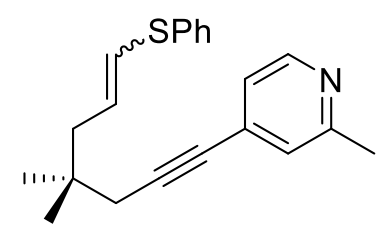

4-(4,4-dimethyl-7-(phenylthio)hept-6-en-1-yn-1-yl)-2-methylpyridine (14)
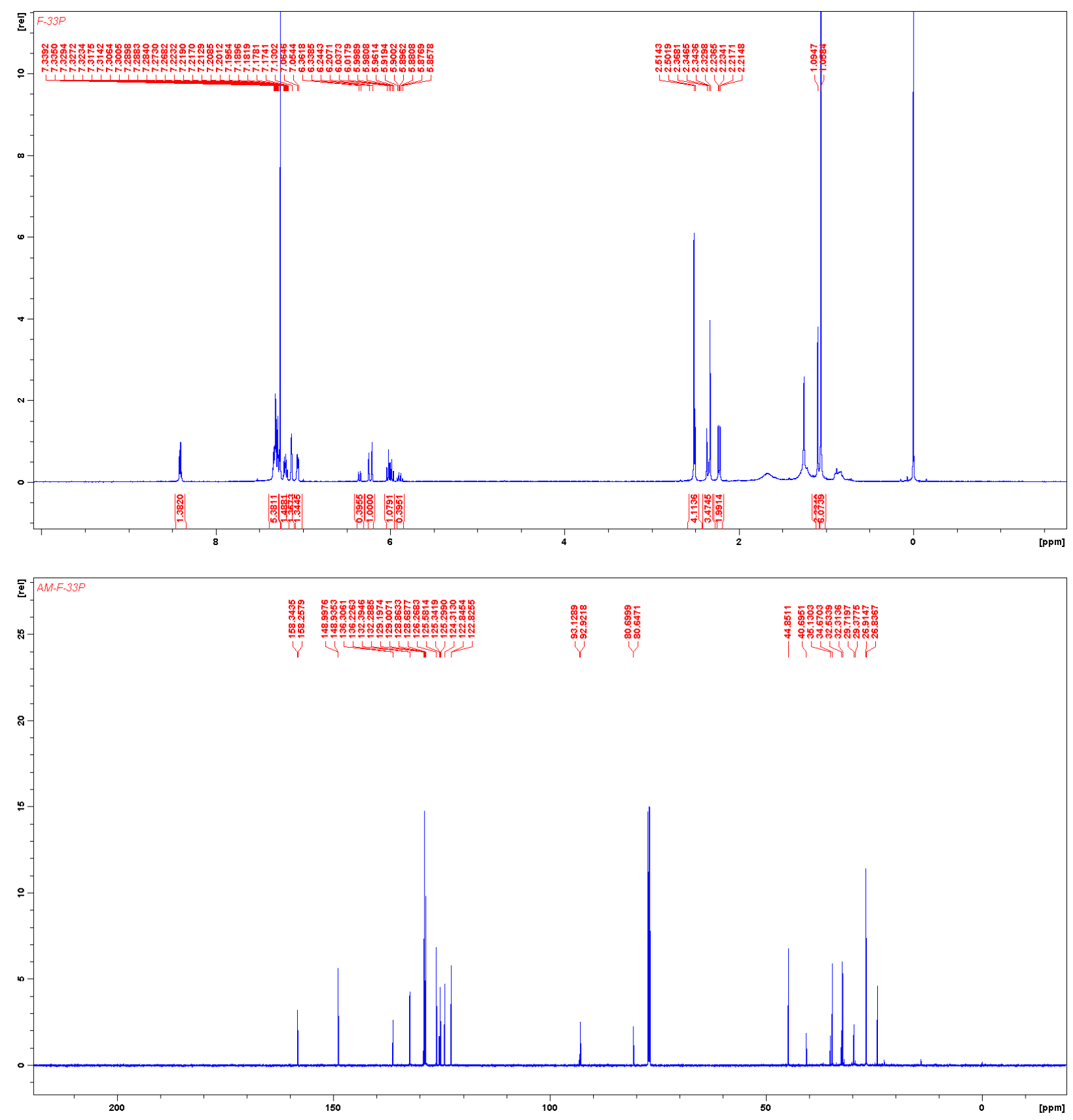


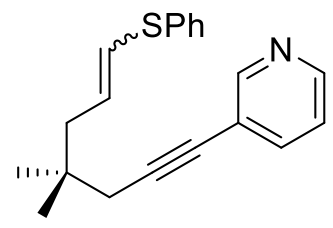

3-(4,4-dimethyl-7-(phenylthio)hept-6-en-1-yn-1-yl)pyridine (16)
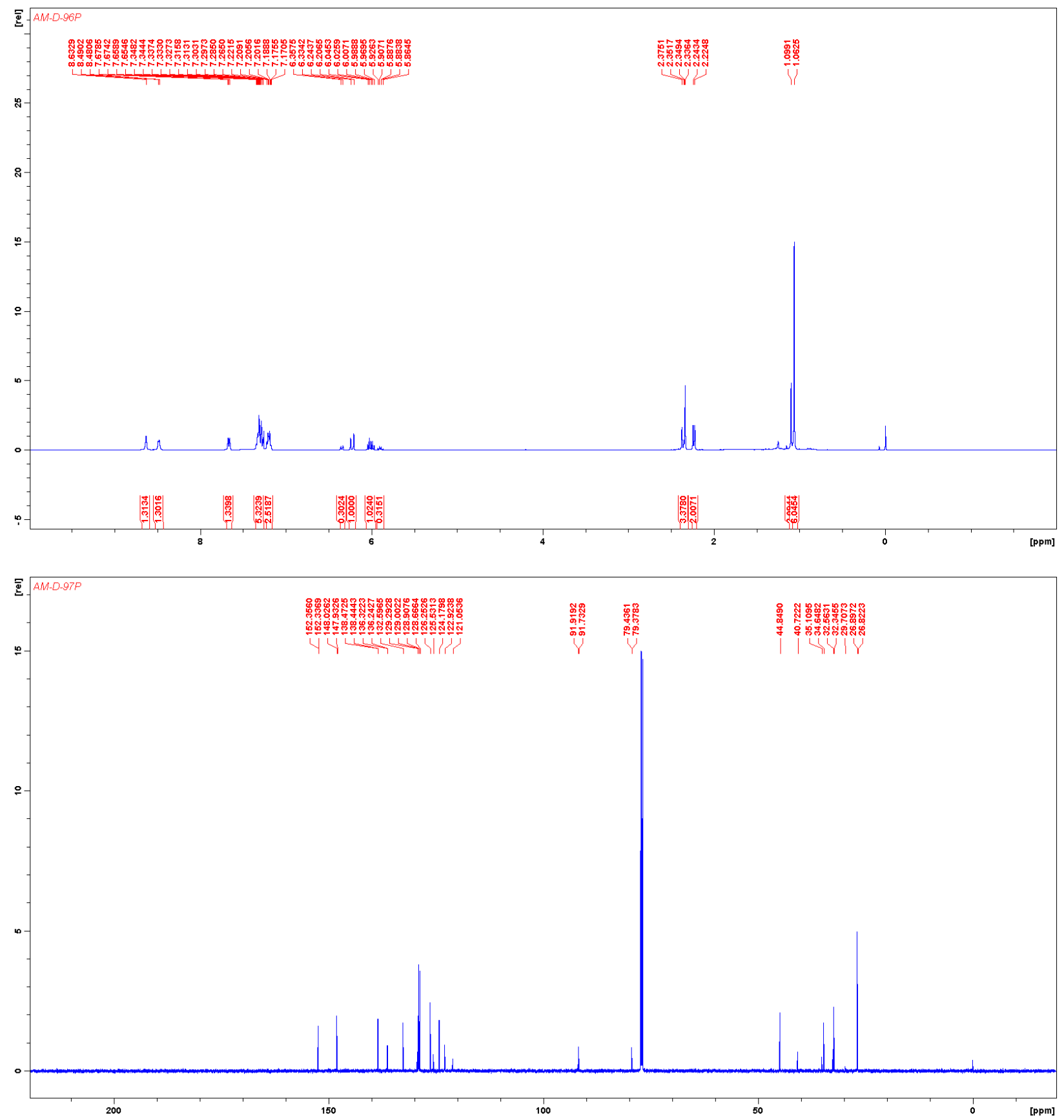
<smiles>CC(C)(CC#Cc1ccncc1Cl)C/C=C/[SbH]c1ccccc1</smiles>

3-chloro-4-(4,4-dimethyl-7-(phenylthio)hept-6-en-1-yn-1-yl)pyridine (18)
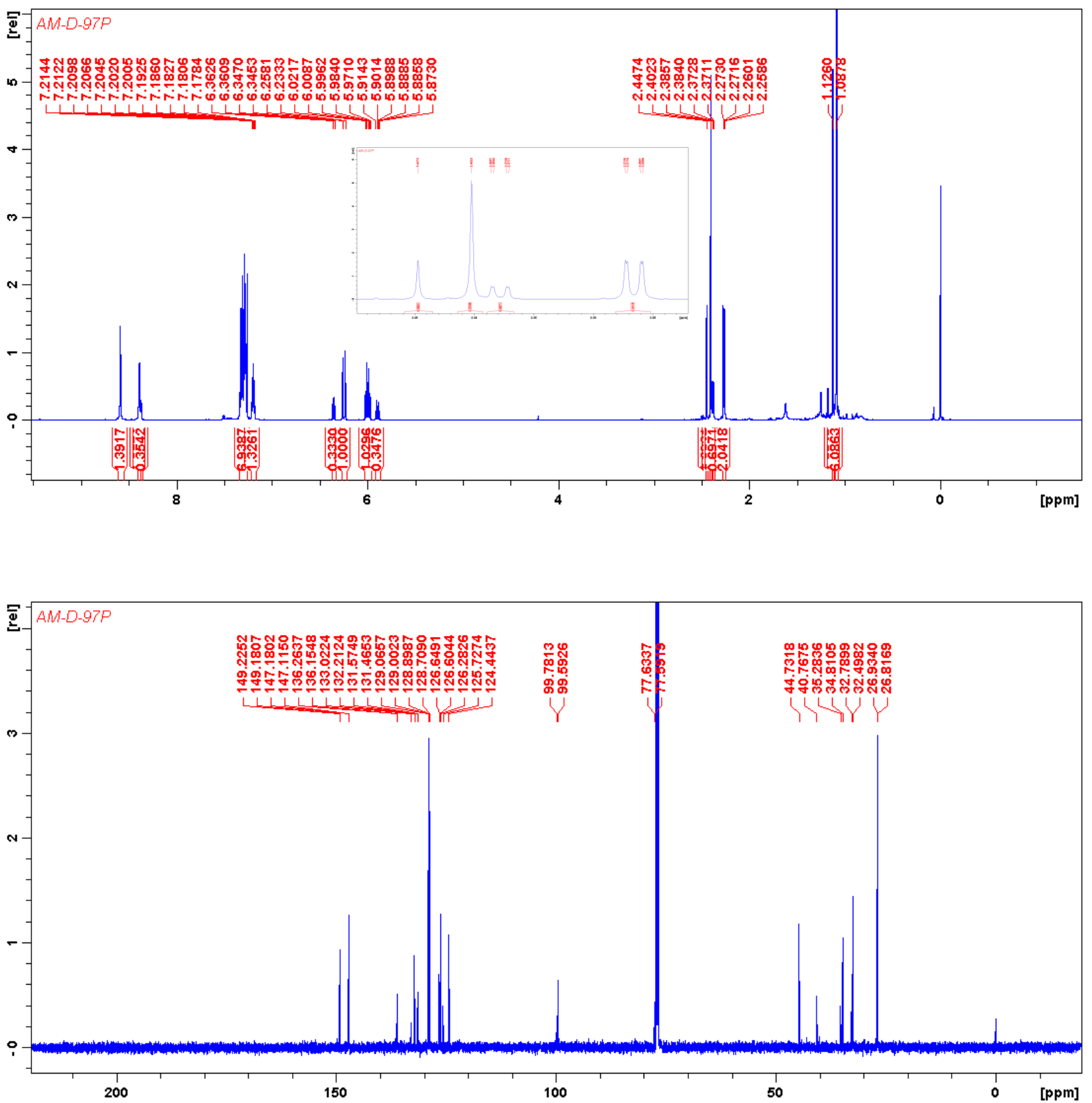
<smiles>CC(C)(CC#Cc1ccnc2ccccc12)C/C=C/[Sb]c1ccccc1</smiles>

4-(4,4-dimethyl-7-(phenylthio)hept-6-en-1-yn-1-yl)quinolone (20)
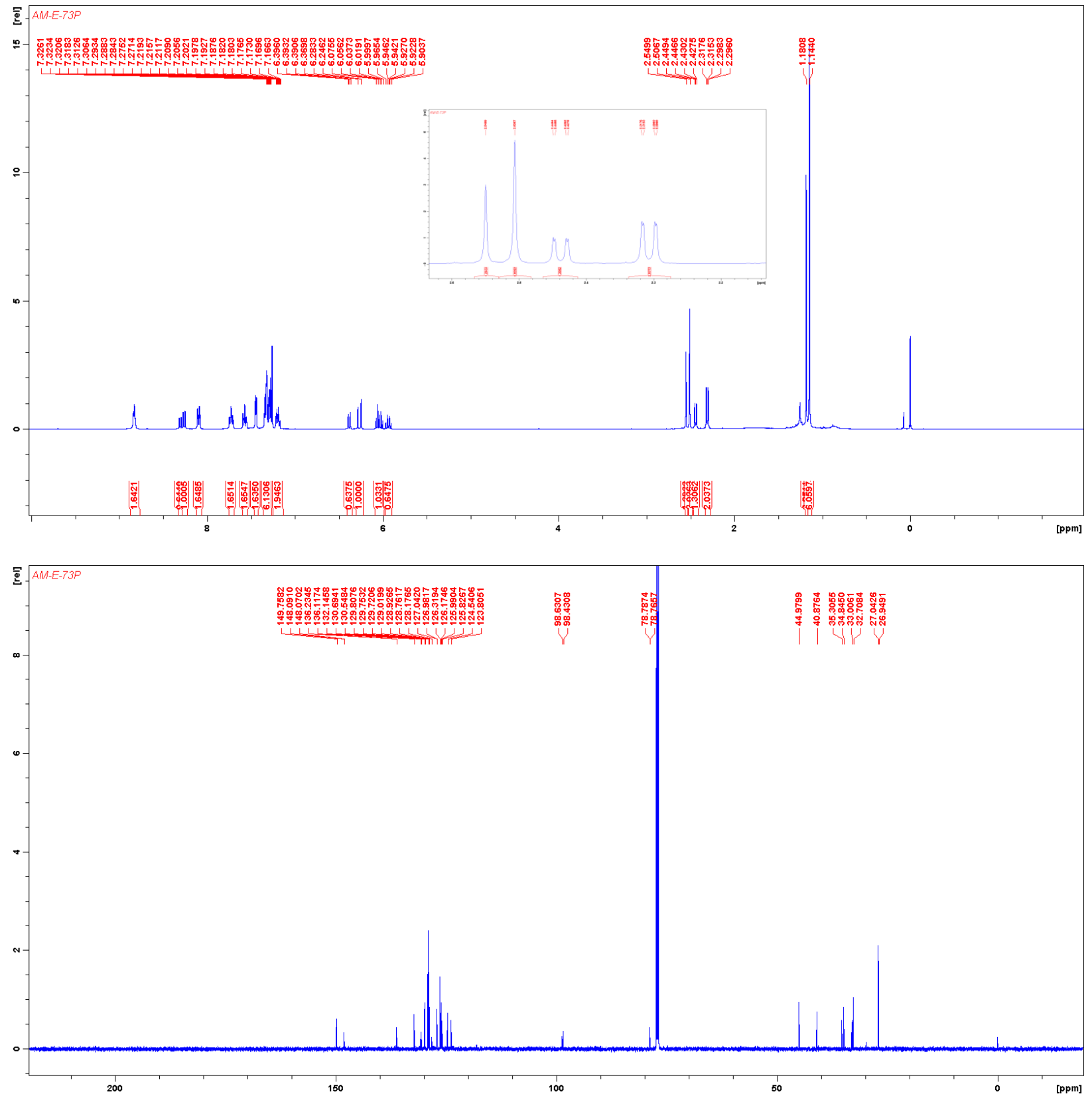
<smiles>CC(C)(CC#Cc1cncc2ccccc12)C/C=C/[Sb]c1ccccc1</smiles>

4-(4,4-dimethyl-7-(phenylthio)hept-6-en-1-yn-1-yl)isoquinoline (22)
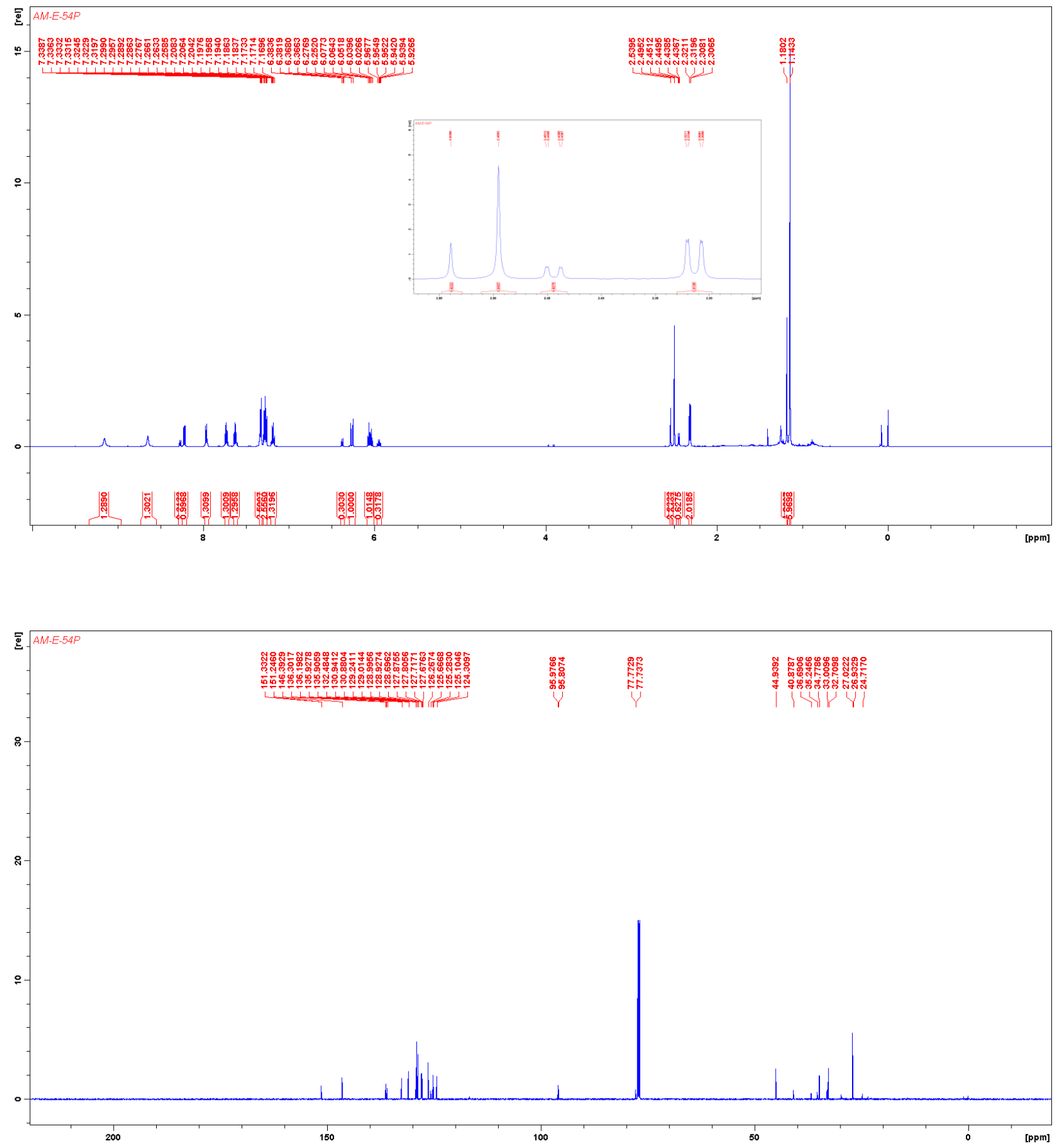
S32

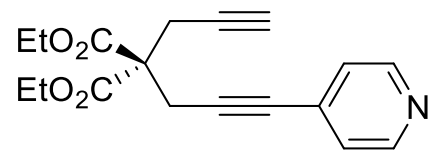

diethyl 2-(prop-2-yn-1-yl)-2-(3-(pyridin-4-yl)prop-2-yn-1-yl)malonate (1)
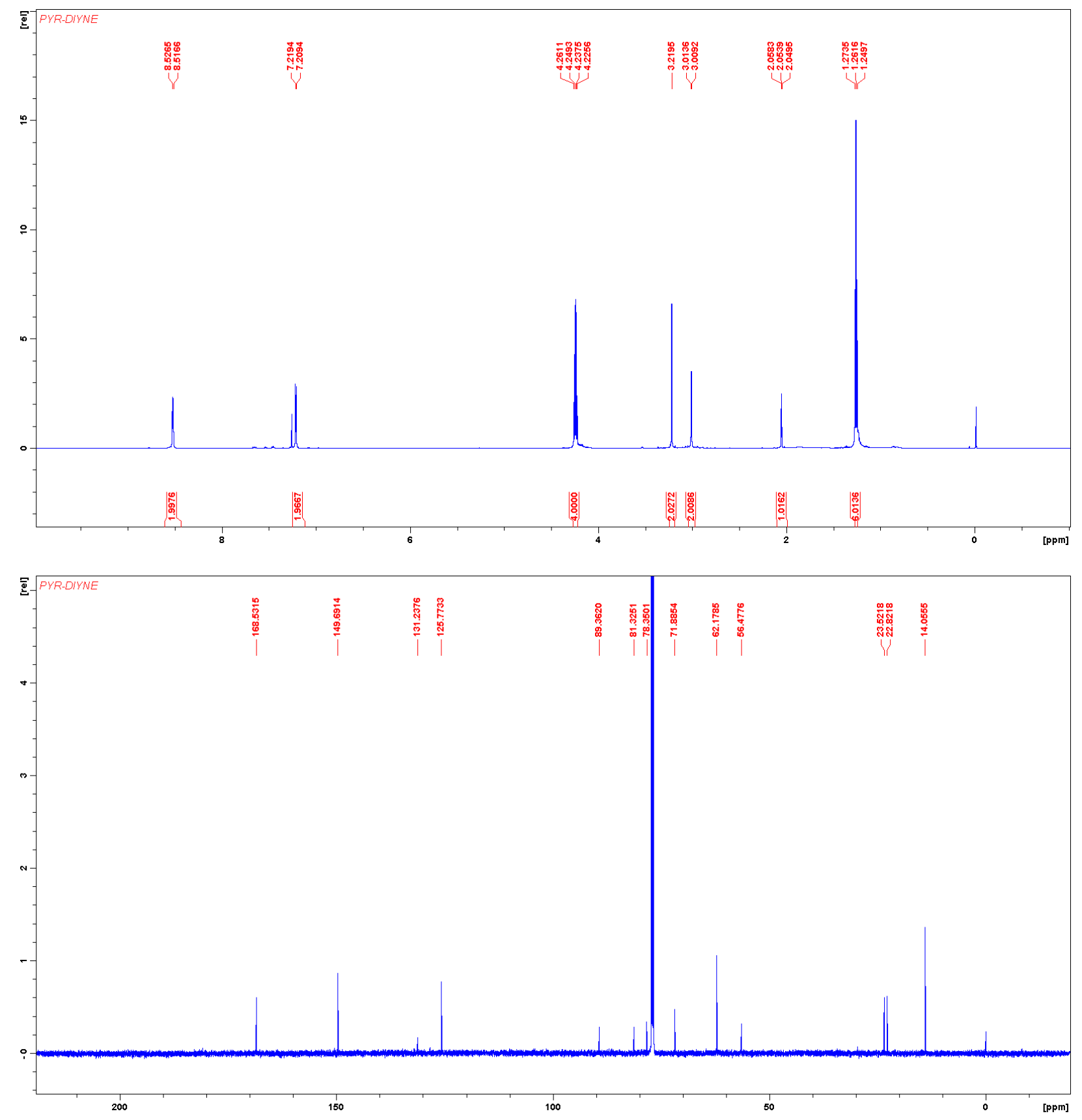
<smiles>CC1(C)Cc2cc3ccncc3cc2C1</smiles>

7,7-dimethyl-7,8-dihydro-6H-cyclopenta[g]isoquinoline (4)
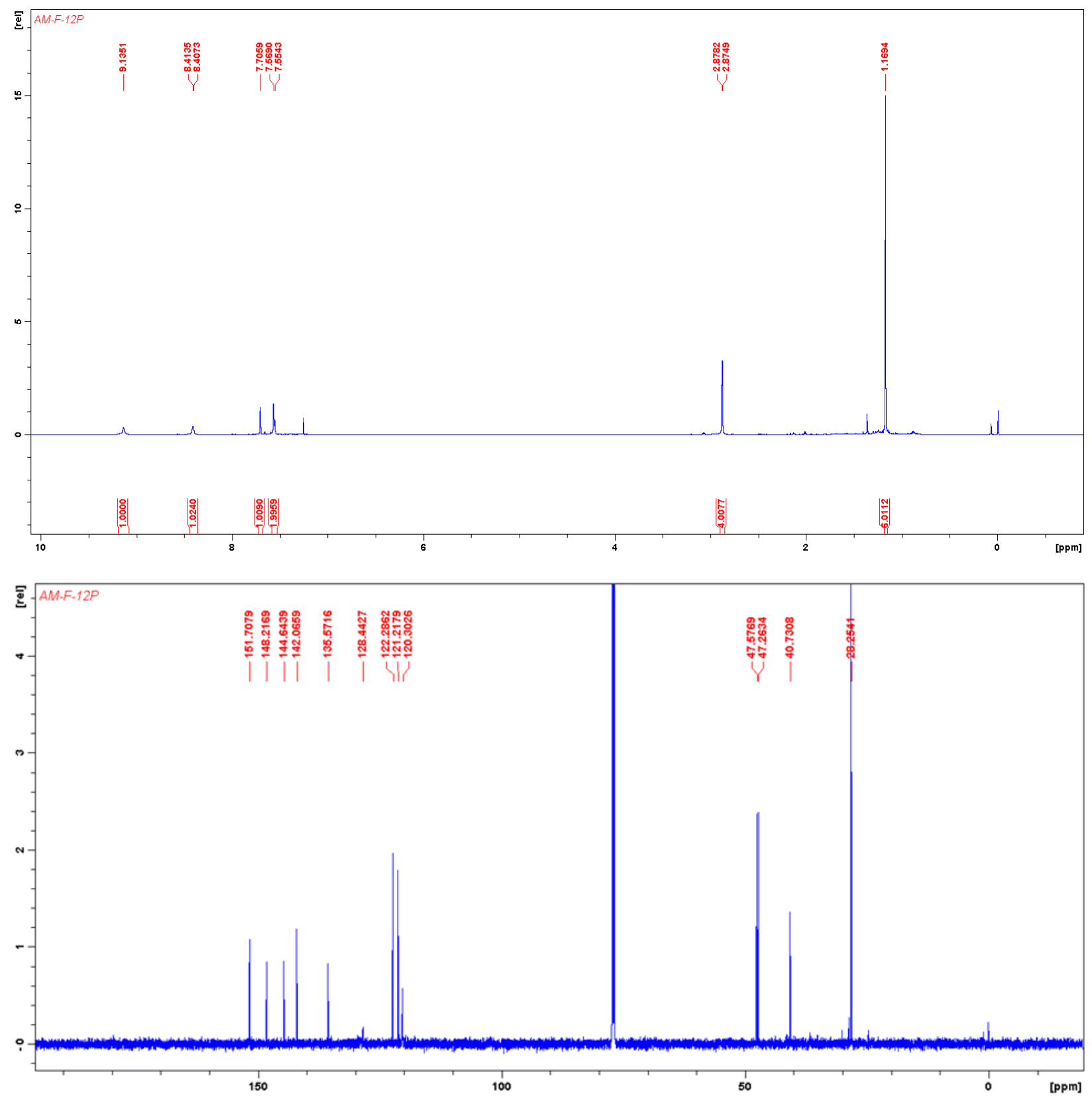
S34

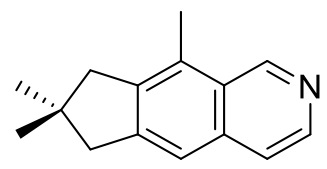

7,7,9-trimethyl-7,8-dihydro-6H-cyclopenta[g]isoquinoline (11)
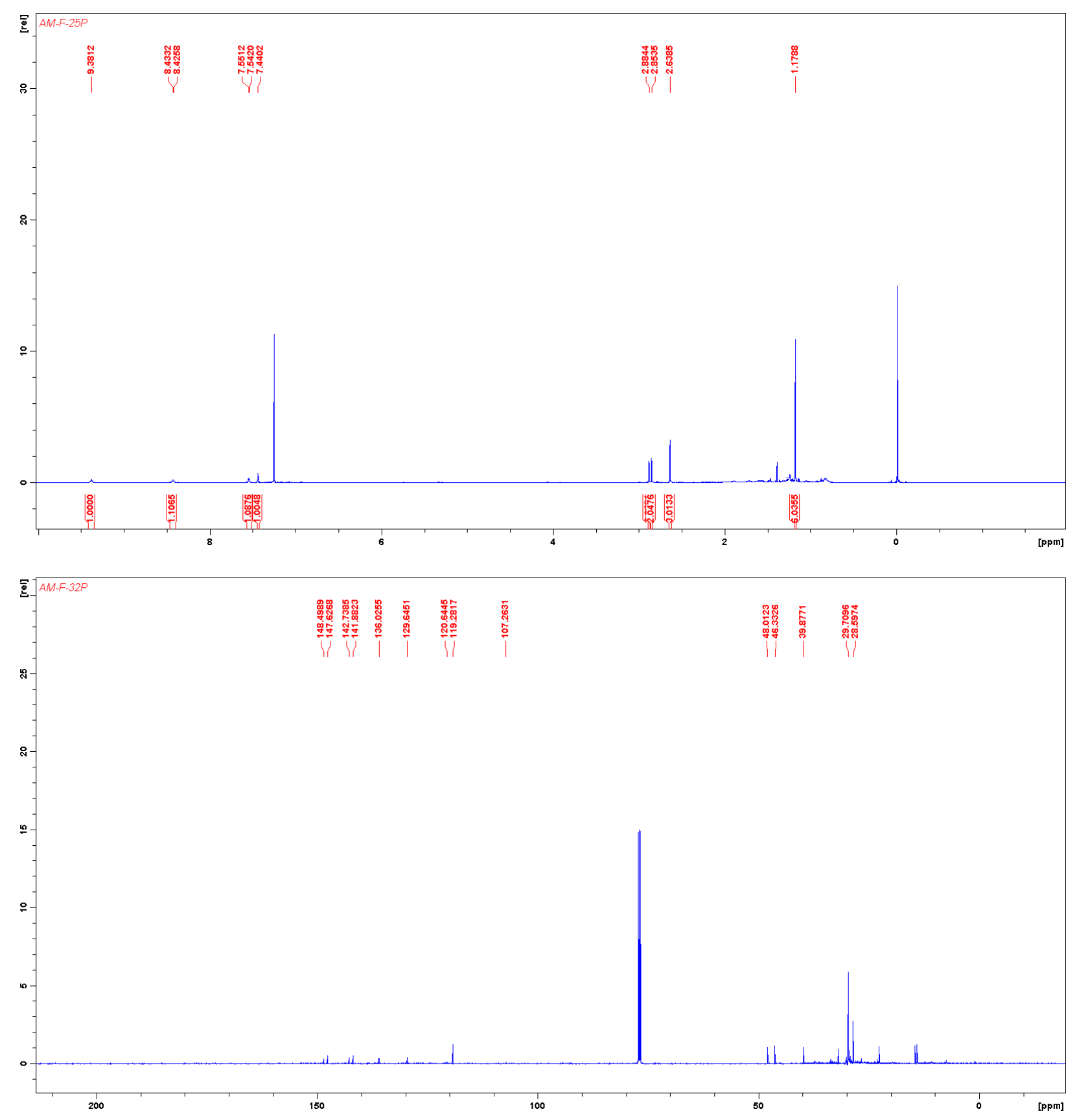


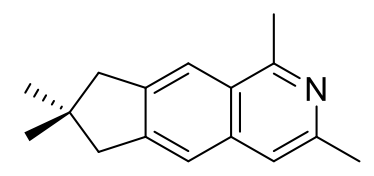

1,3,7,7-tetramethyl-7,8-dihydro-6H-cyclopenta[g]isoquinoline (13)
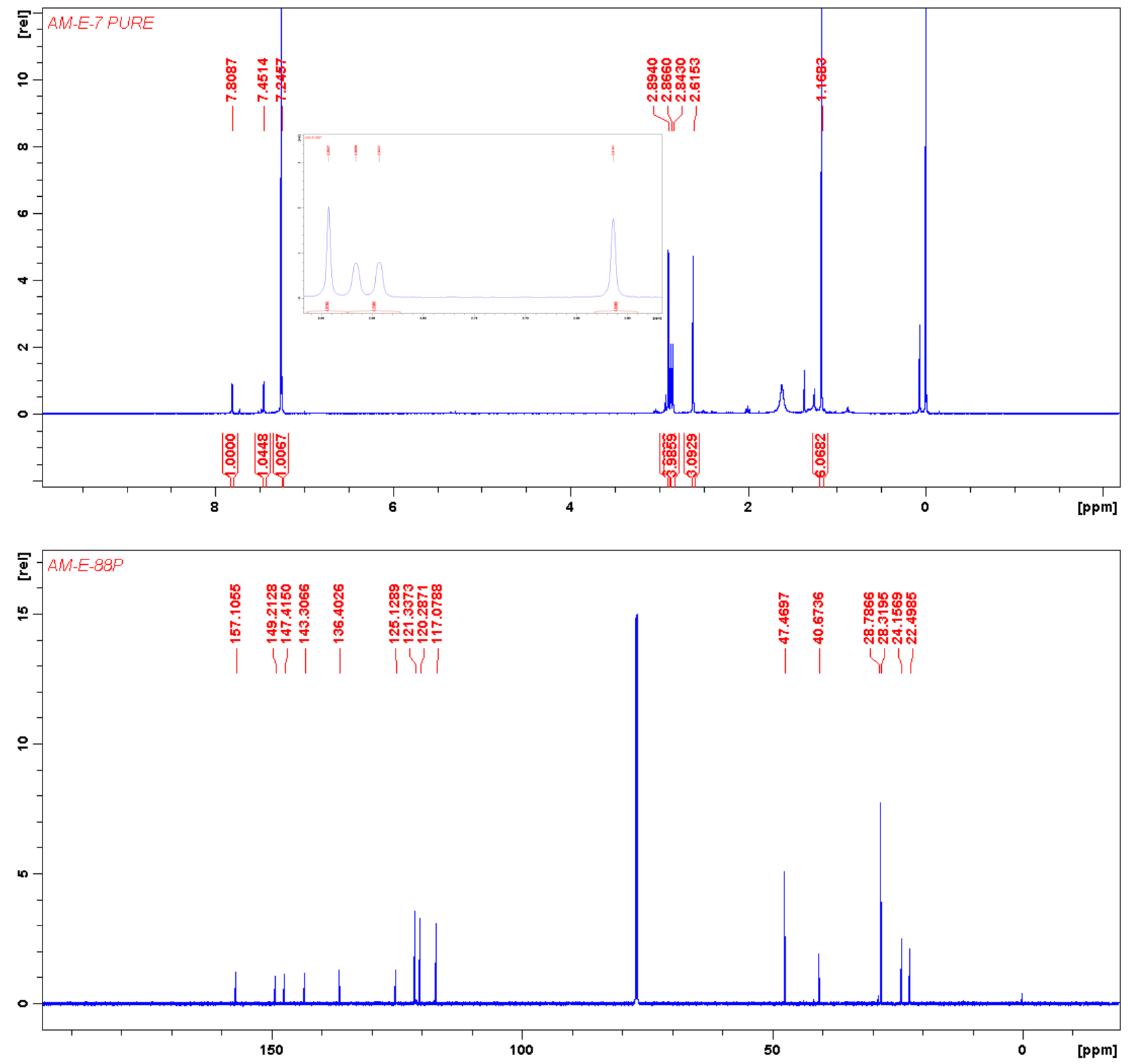
<smiles>Cc1cc2cc3c(cc2cn1)CC(C)(C)C3</smiles>

1,7,7-trimethyl-7,8-dihydro-6H-cyclopenta[g]isoquinoline (15a)

3,7,7-trimethyl-7,8-dihydro-6H-cyclopenta[g]isoquinoline (15b)
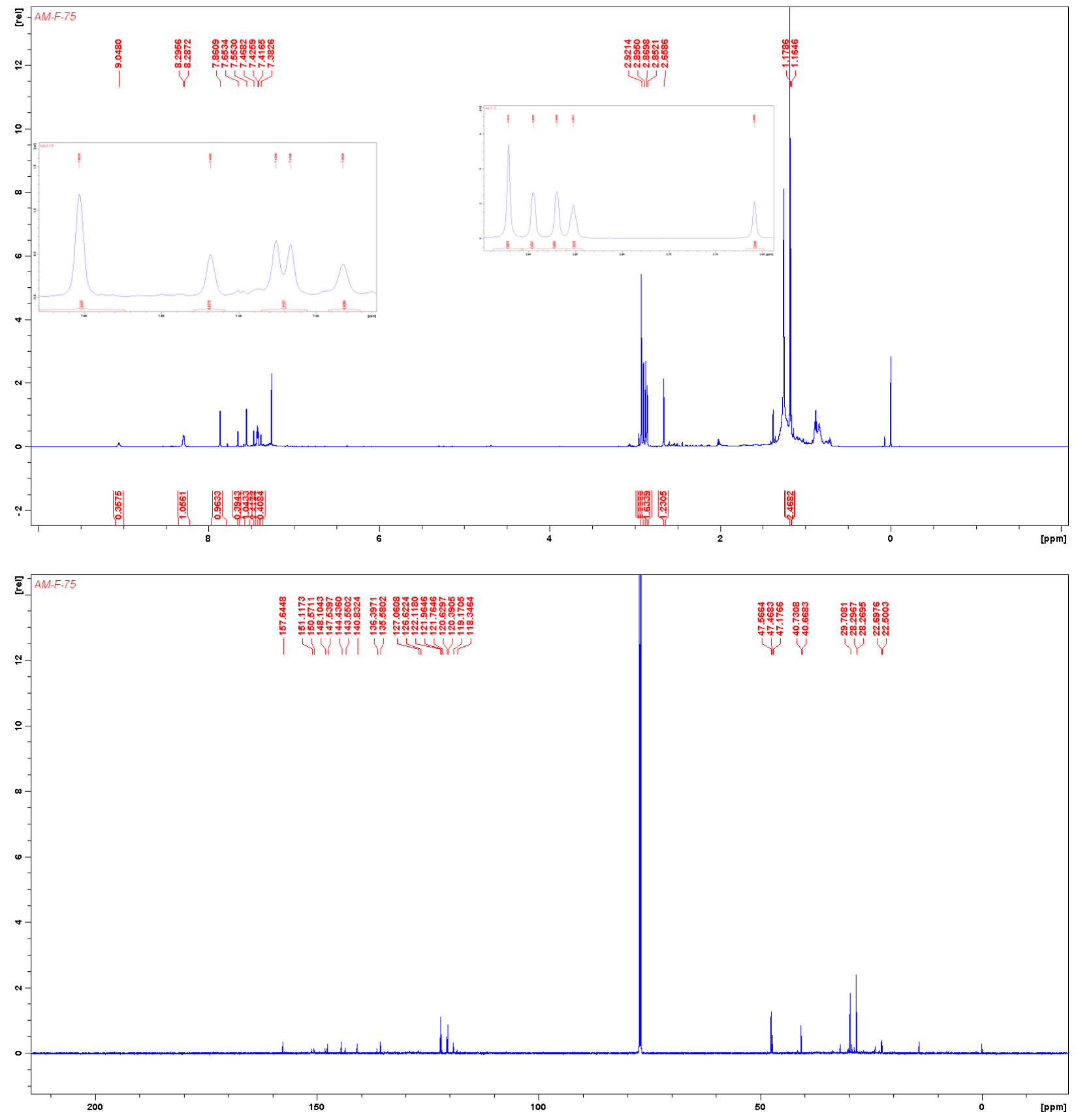


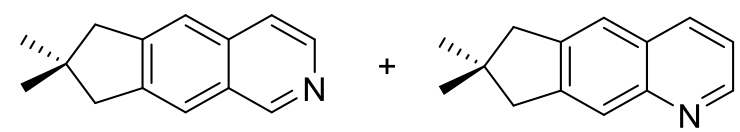

7,7-dimethyl-7,8-dihydro-6H-cyclopenta[g]isoquinoline (17a)

7,7-dimethyl-7,8-dihydro-6H-cyclopenta[g]quinolone (17b)
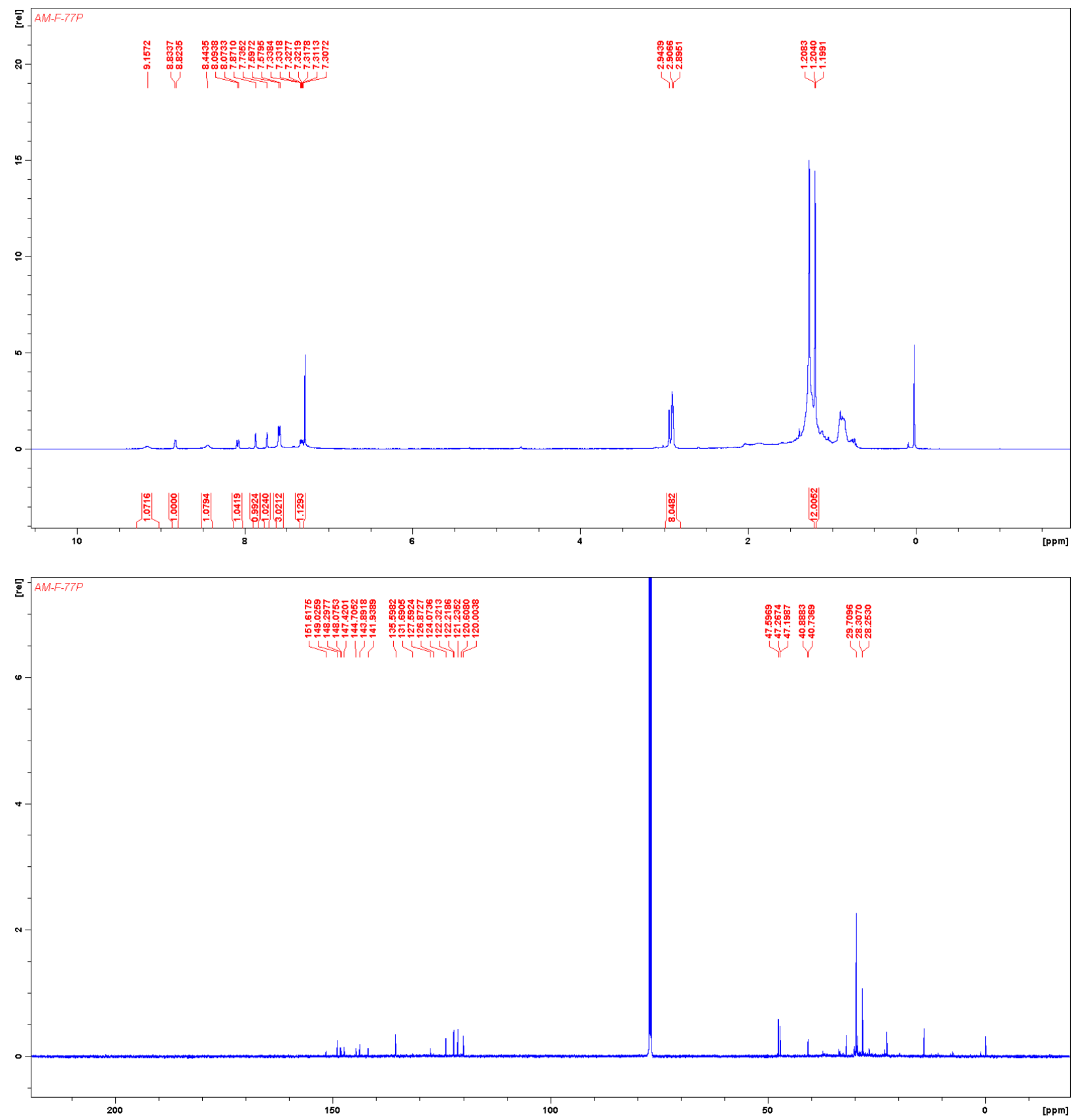
<smiles>CC1(C)Cc2cc3cncc(Cl)c3cc2C1</smiles>

4-chloro-7,7-dimethyl-7,8-dihydro-6H-cyclopenta[g]isoquinoline (19)
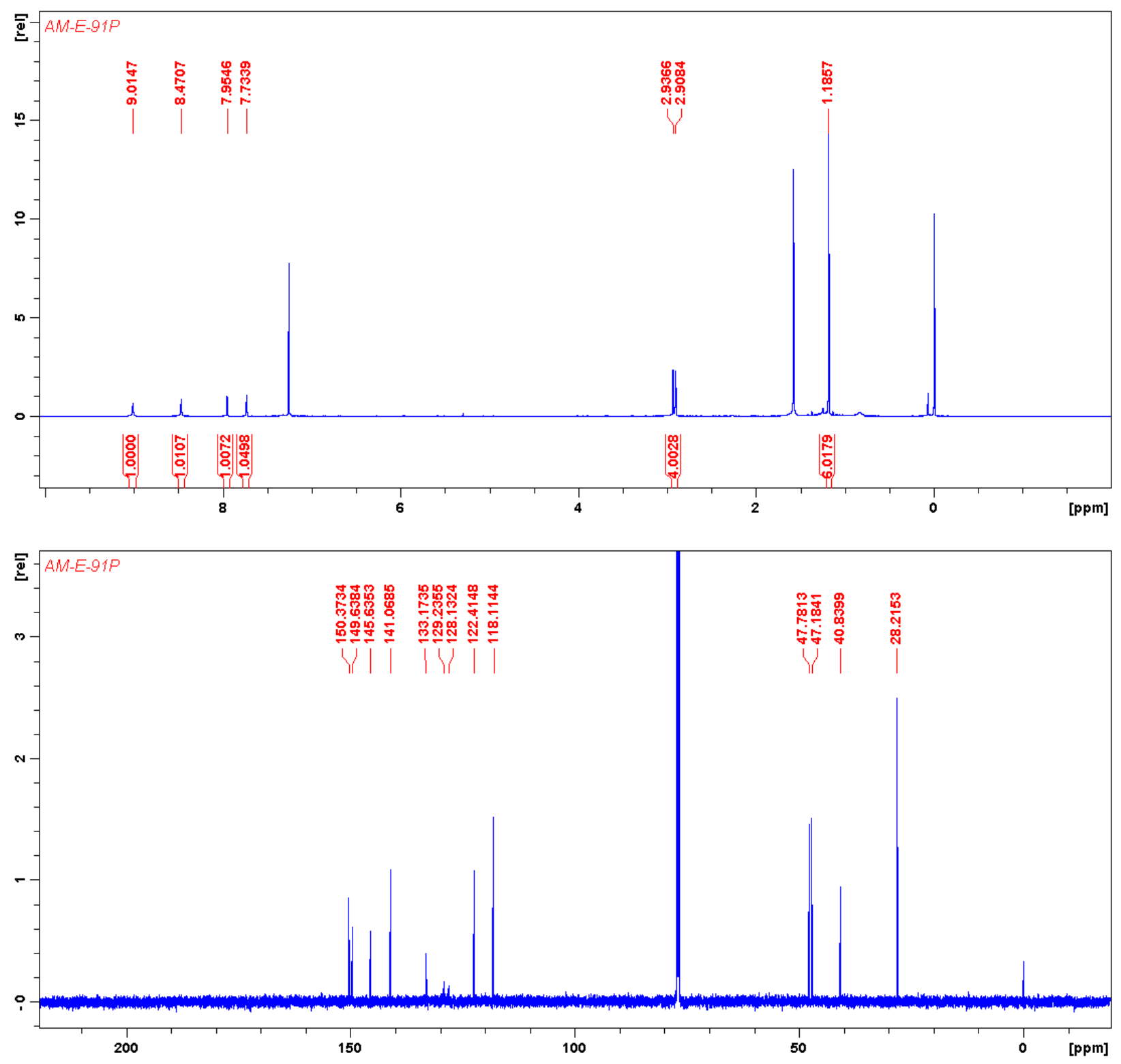
<smiles>CC1(C)Cc2cc3cnc4ccccc4c3cc2C1</smiles>

9,9-dimethyl-9,10-dihydro-8H-cyclopenta[j]phenanthridine (21)
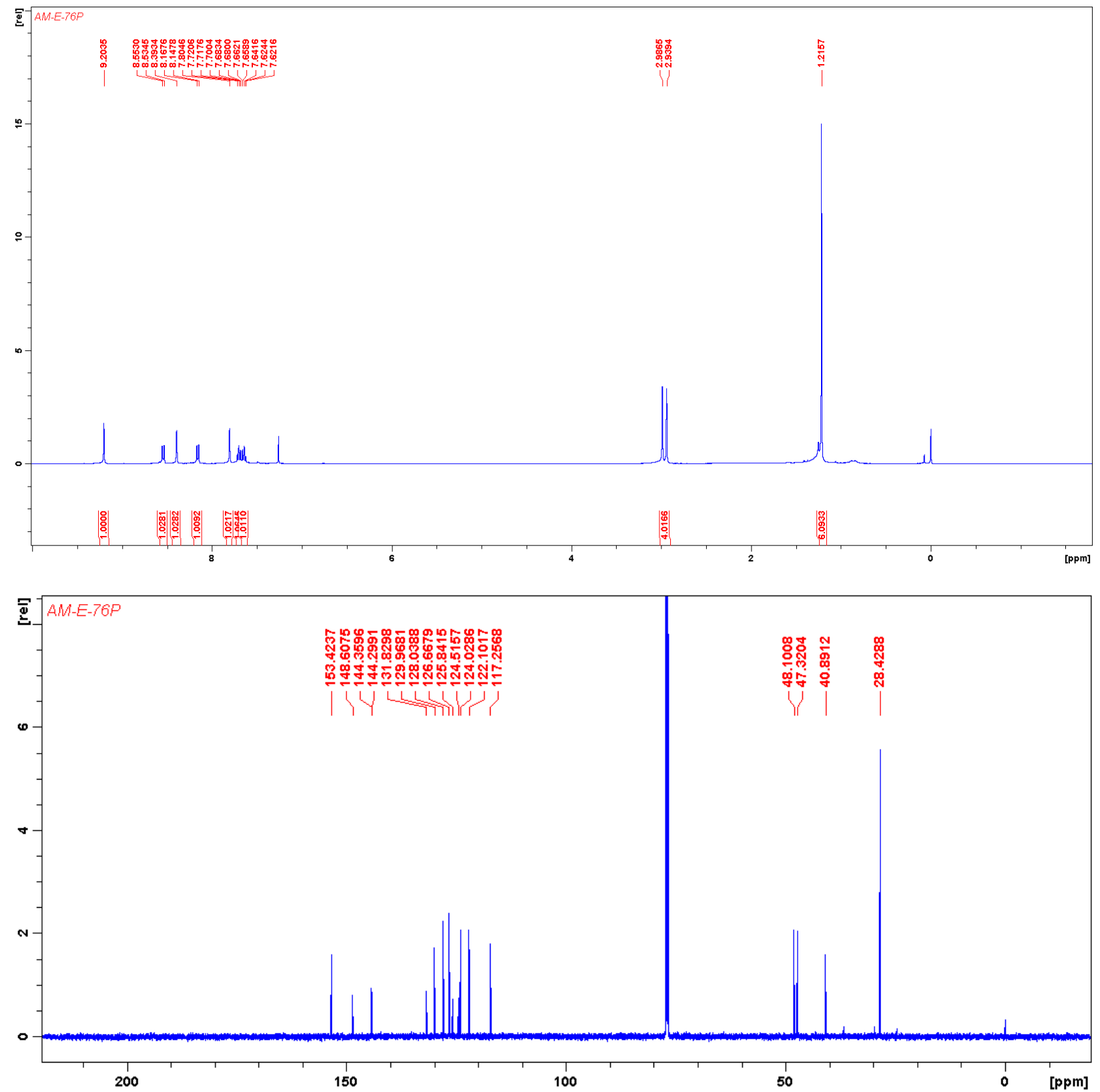
<smiles>CC1(C)Cc2cc3ncc4ccccc4c3cc2C1</smiles>

9,9-dimethyl-9,10-dihydro-8H-cyclopenta[b]phenanthridine (23)
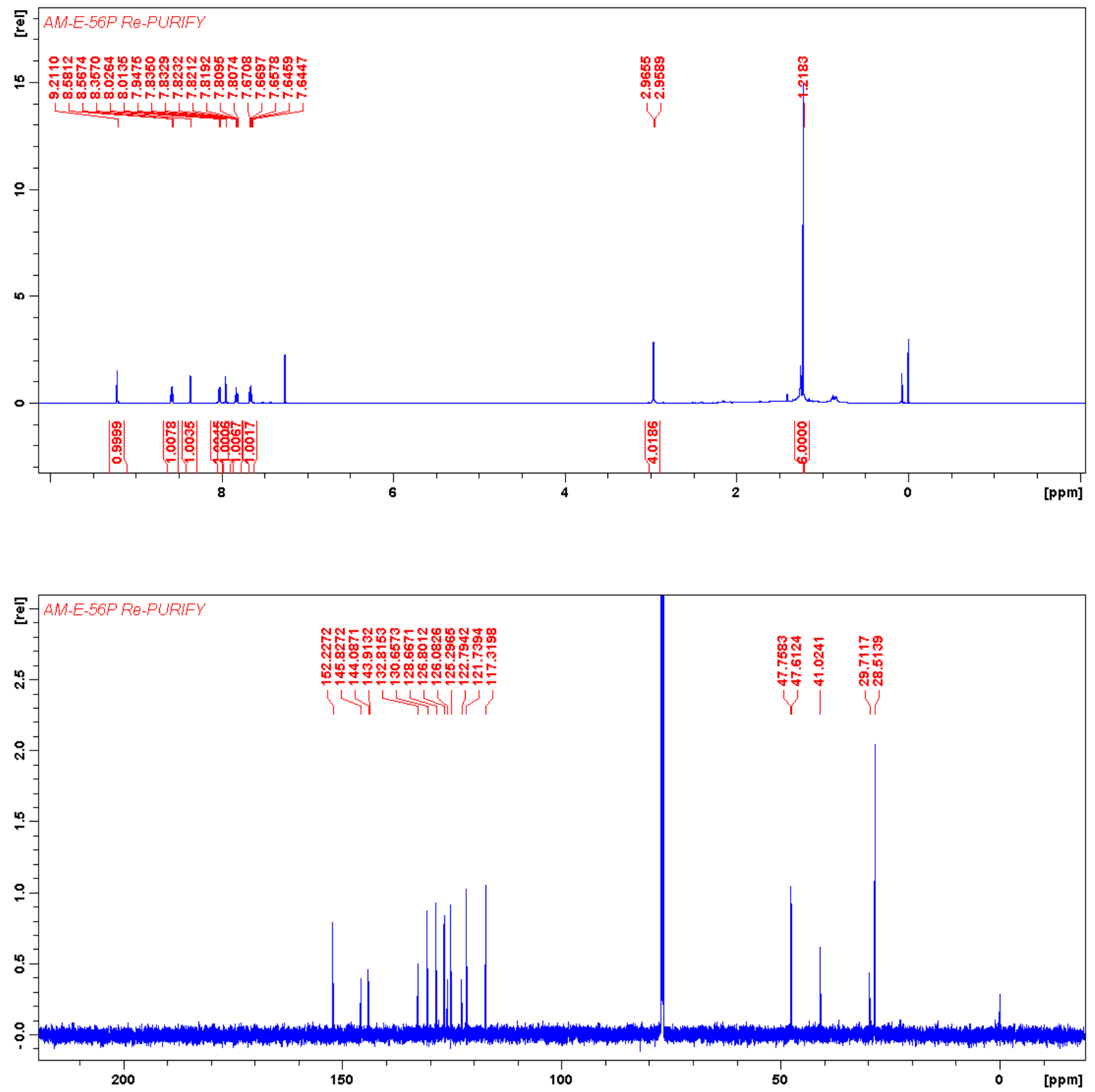
<smiles>CC1(C)Cc2c(c(Br)c3cnccc3c2Br)C1</smiles>

5,9-dibromo-7,7-dimethyl-7,8-dihydro-6H-cyclopenta[g]isoquinoline (24)
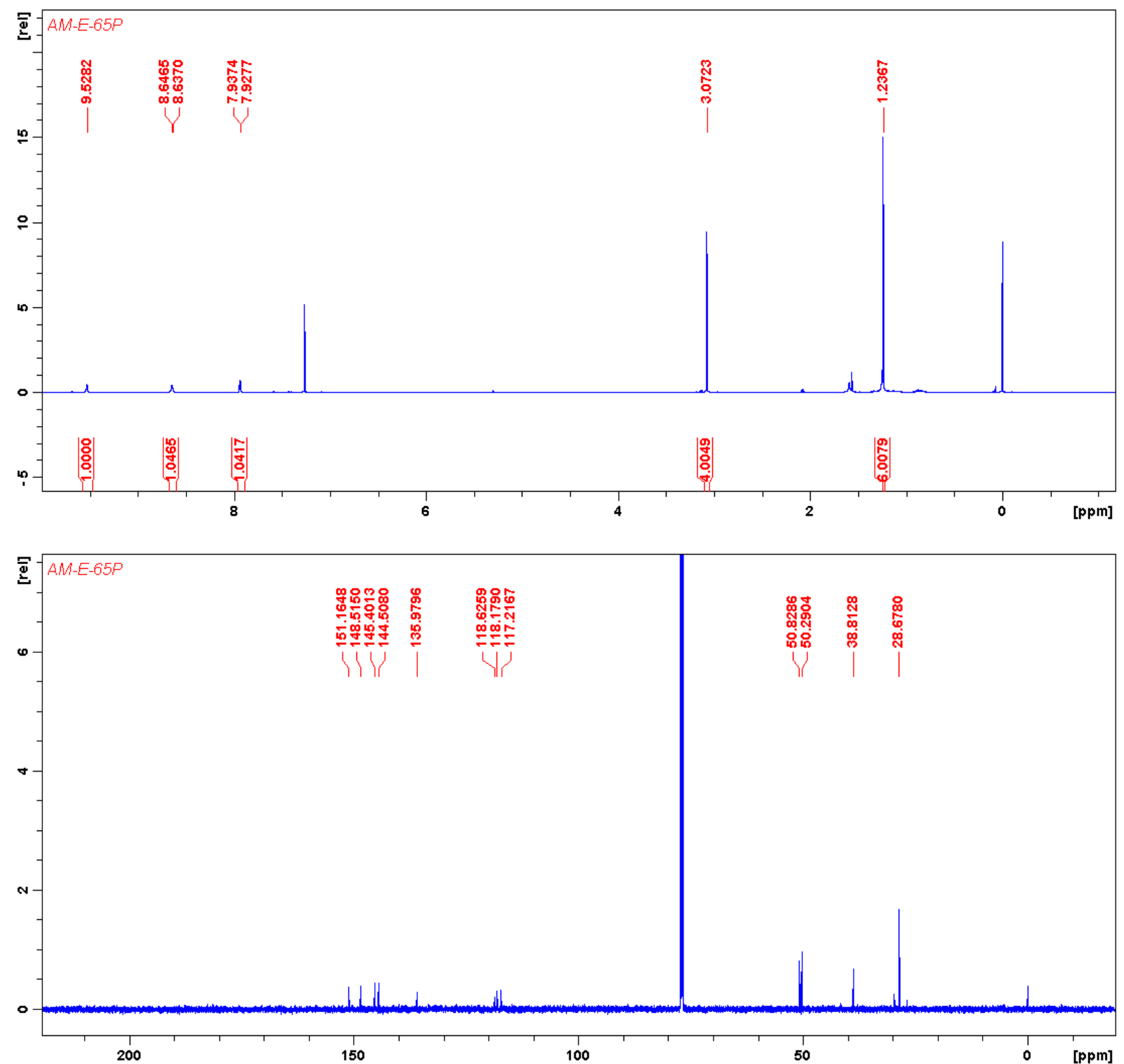
<smiles>Cc1c2c(c(Br)c3ccncc13)CC(C)(C)C2</smiles>

5-bromo-7,7,9-trimethyl-7,8-dihydro-6H-cyclopenta[g]isoquinoline (25)
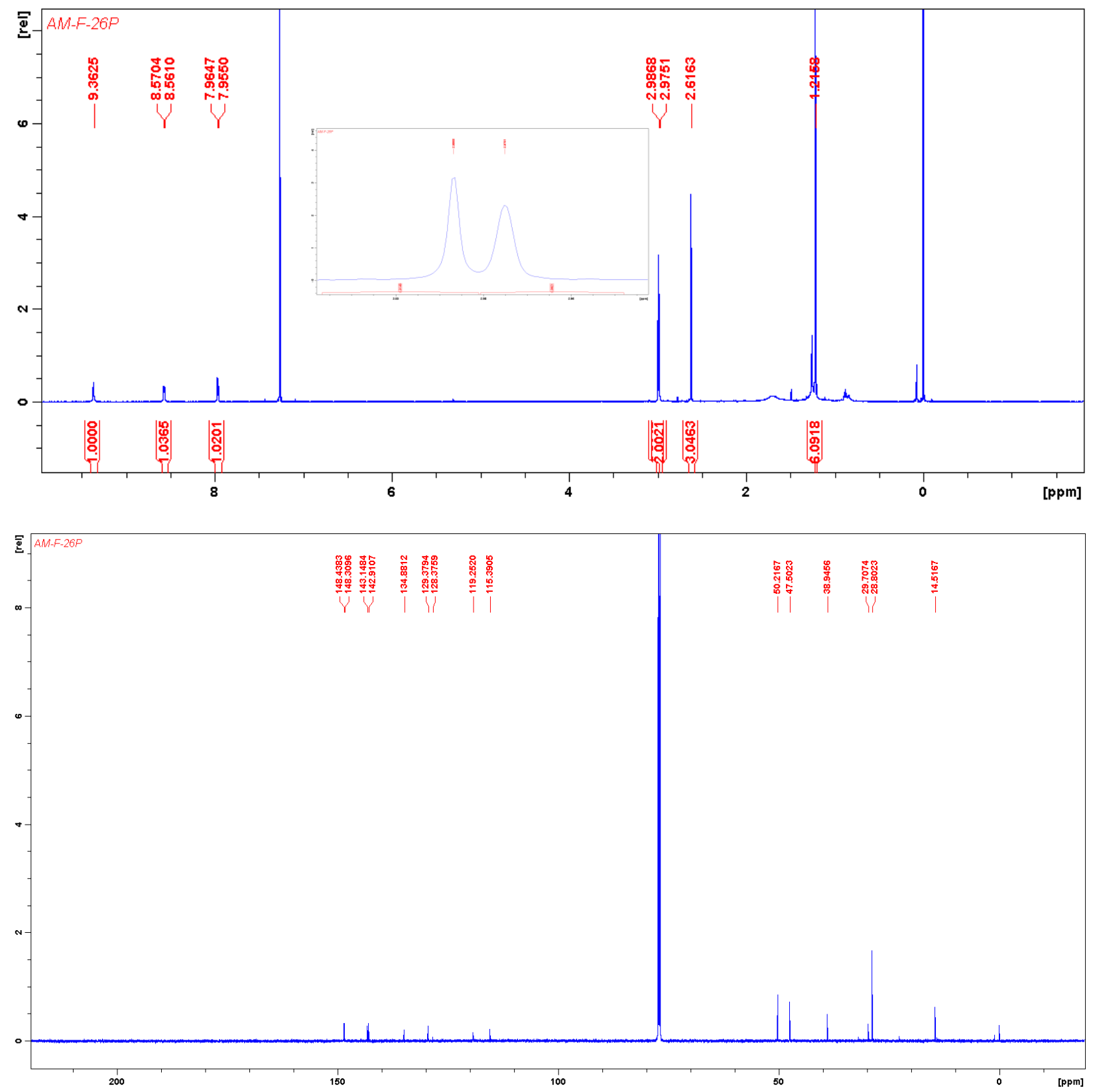
<smiles>Cc1cc2cc3c(cc2c(CC(O)c2ccc(Br)cc2)n1)CC(C)(C)C3</smiles>

1-(4-bromophenyl)-2-(3,7,7-trimethyl-7,8-dihydro-6H-cyclopenta[g]isoquinolin-1-yl)ethan-1-ol (26)
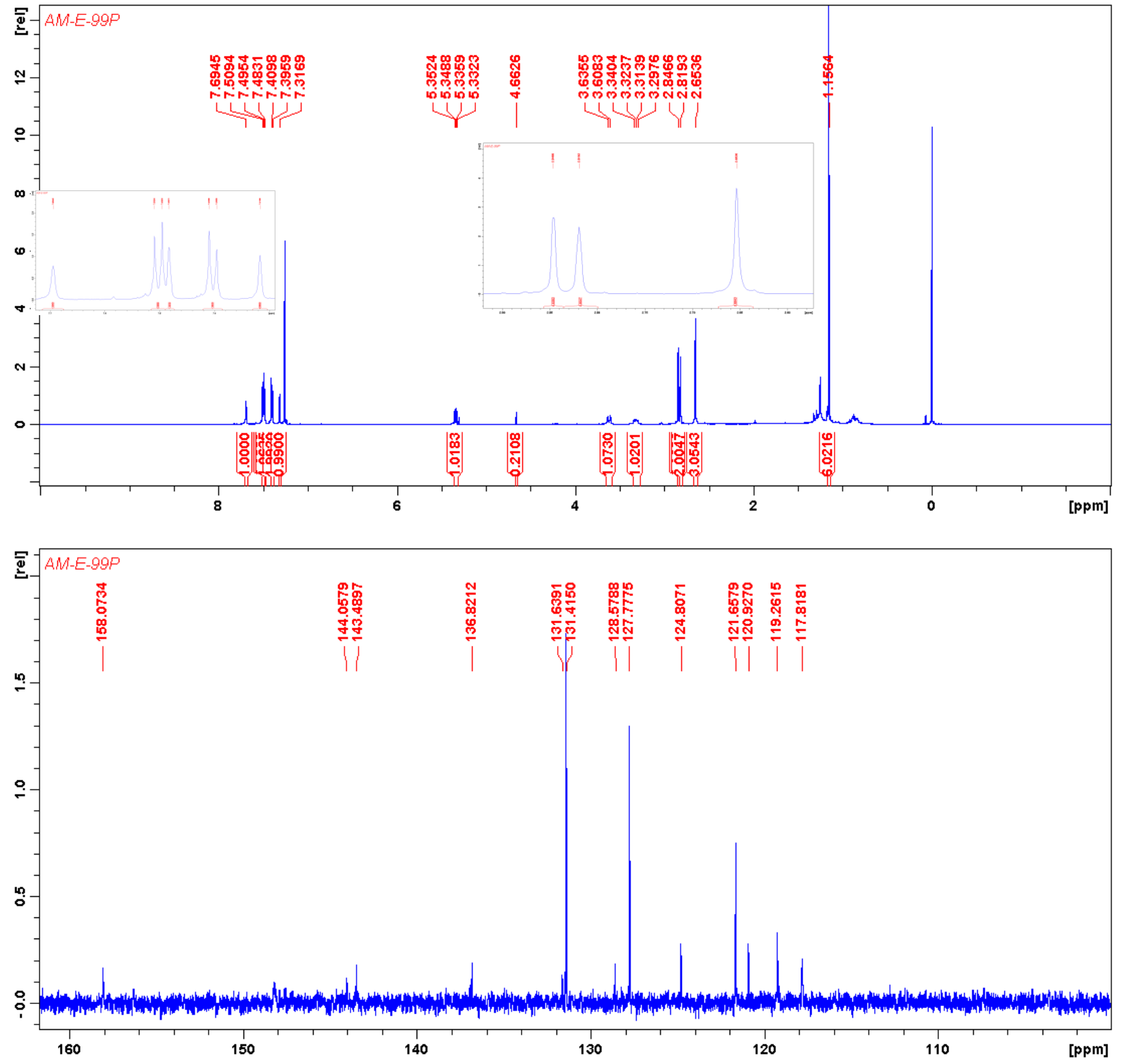


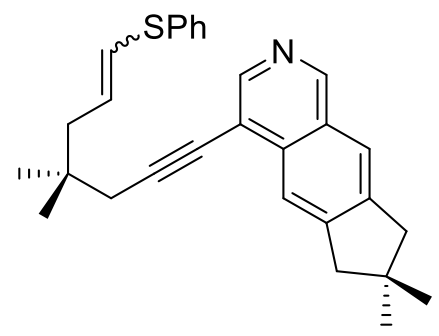

4-(4,4-dimethyl-7-(phenylthio)hept-6-en-1-yn-1-yl)-7,7-dimethyl-7,8-dihydro-6Hcyclopenta[g]isoquinoline (27)
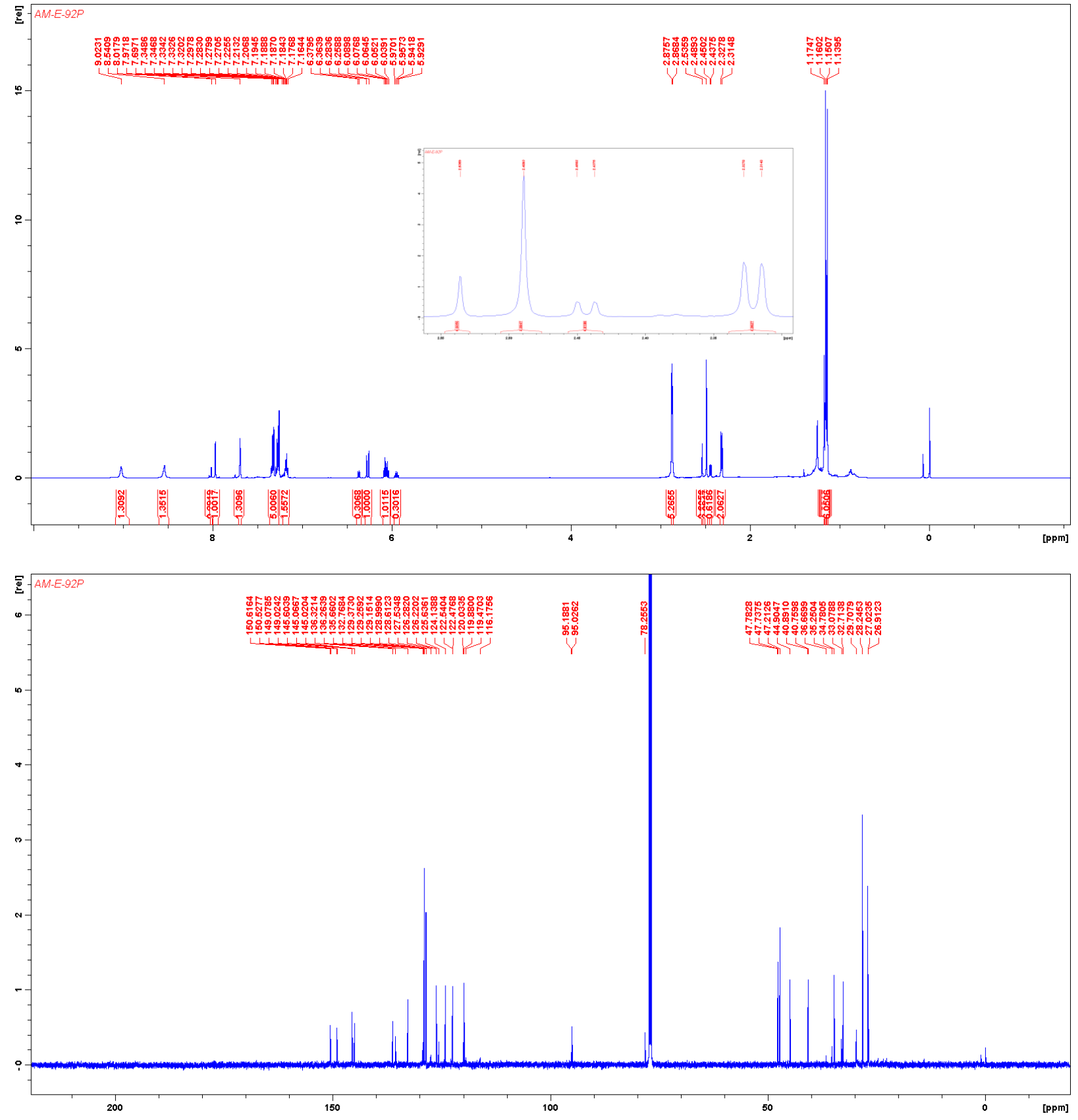


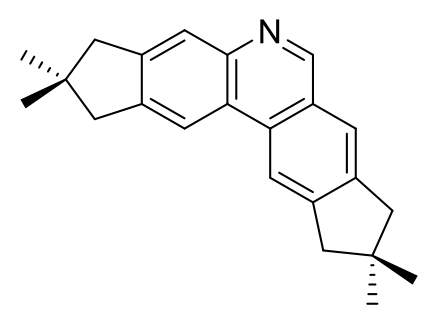

2,2,9,9-tetramethyl-1,2,3,8,9,10-hexahydrodicyclopenta[b,j]phenanthridine (28)
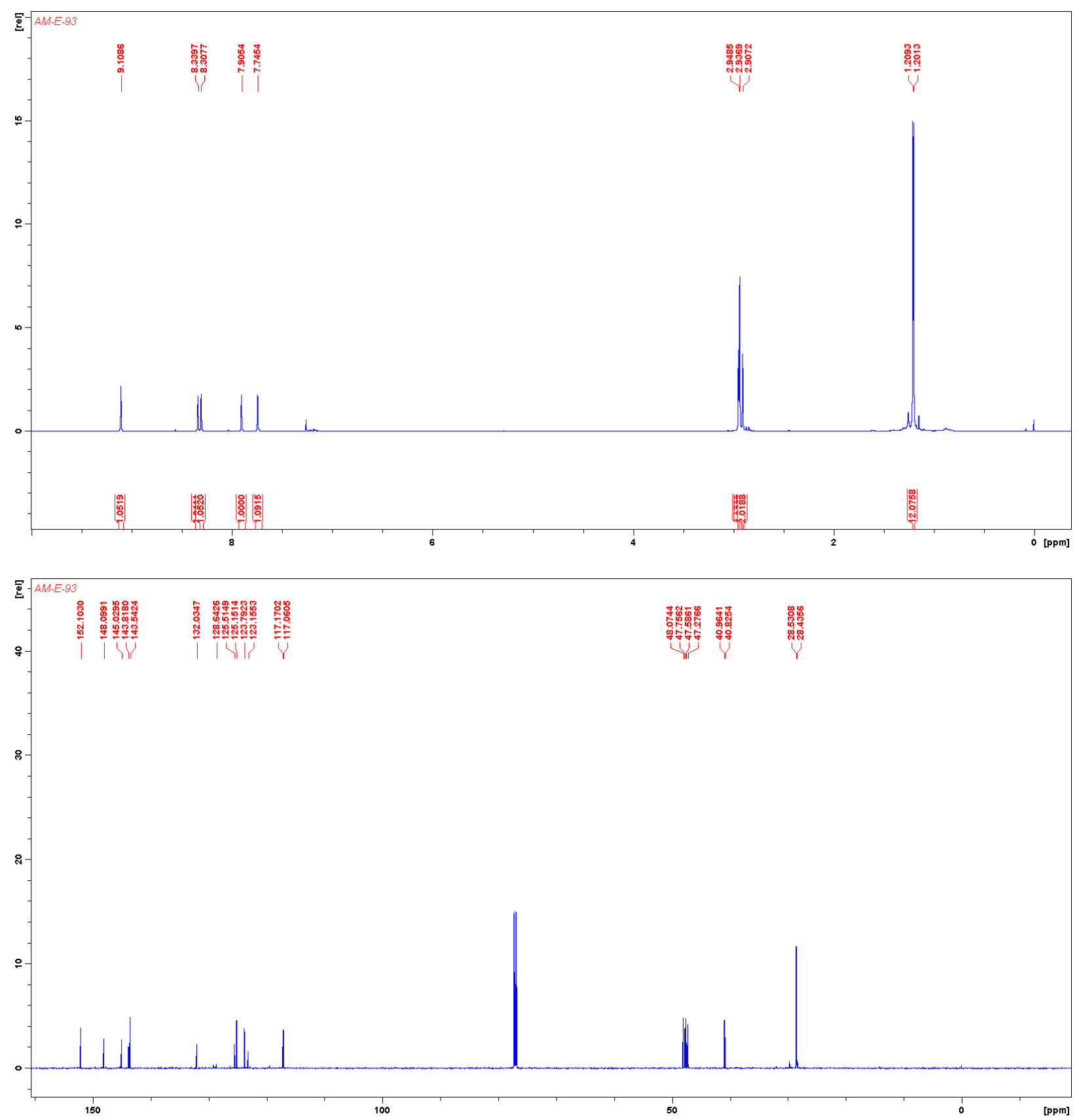
XII. Absorption and Emission of 21, 23, and 28

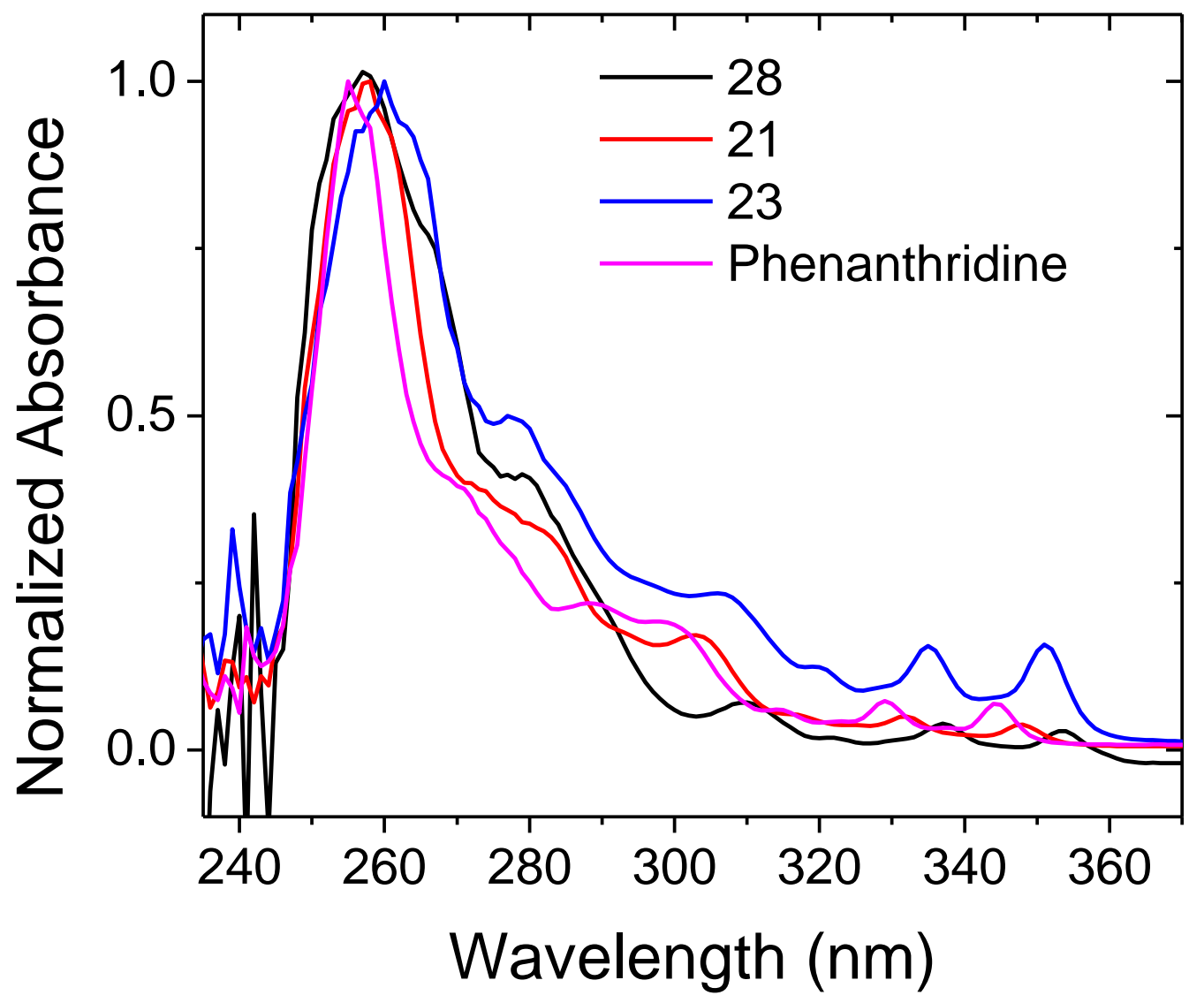




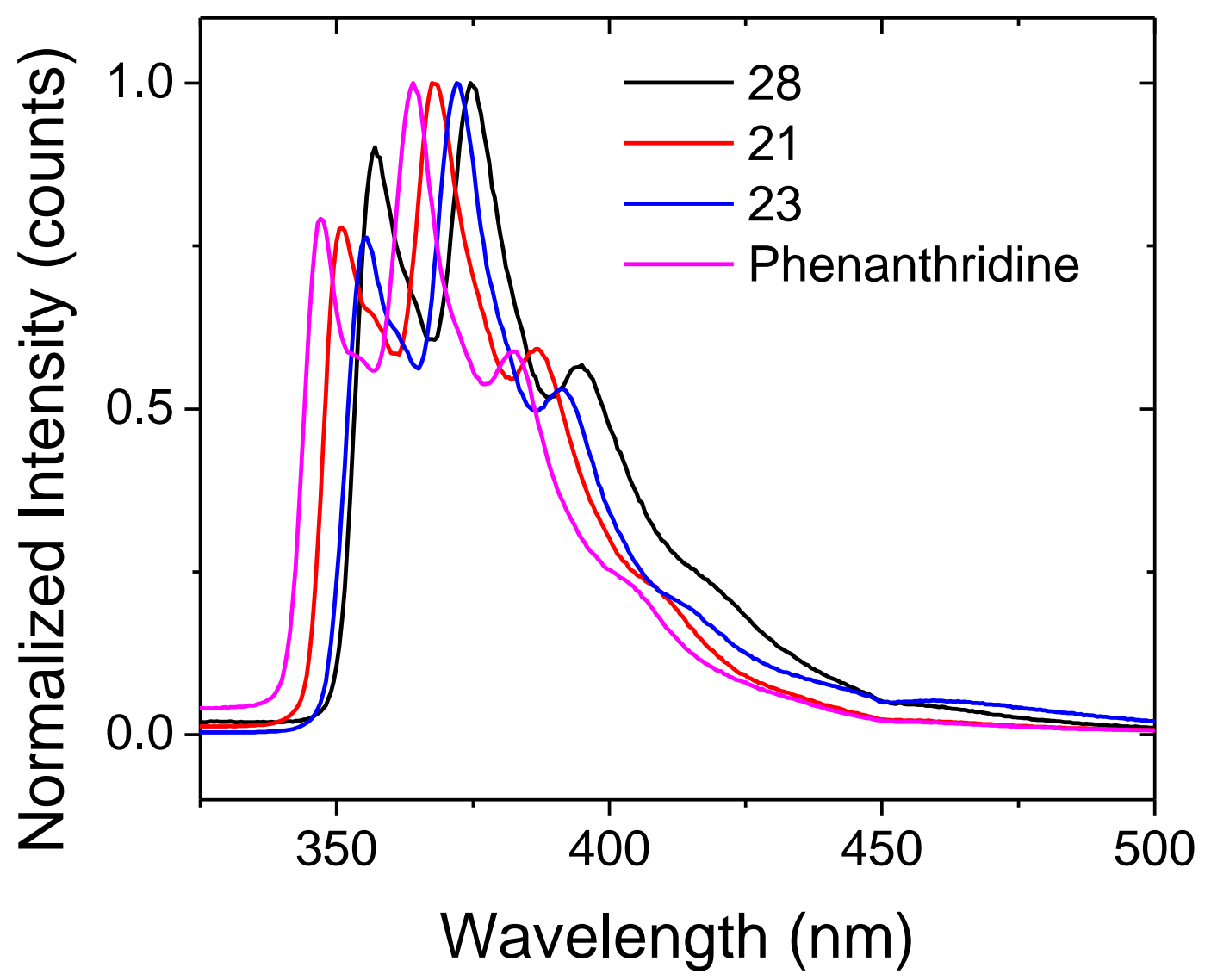

\section{X-ray Crystallography of 28}

\section{(a)}

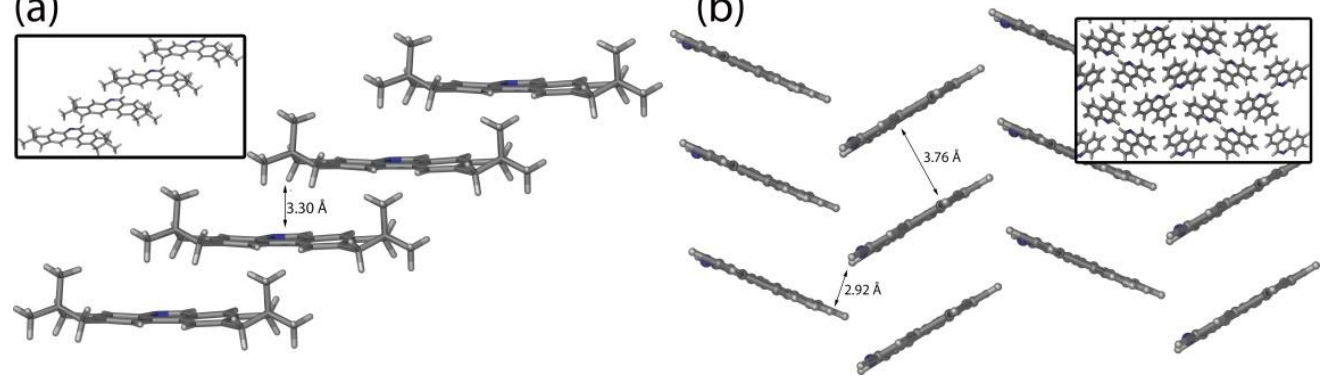

Crystal packing: (a) gem-dimethylcyclopentane-fused phenanthridine $\mathbf{2 8}$ packs in a loosely staggered arrangement whereas phenanthridine itself (b) packs in a herringbone pattern, with edge-to-face interactions at a distance of $2.92 \AA$.

Methods: Single crystals of $\mathbf{2 8}$ were grown via sublimation in a thick-walled Pyrex tube under dynamic vacuum (100 mTorr) at $90 \mathrm{C}$ over 2 days. 
For single-crystal X-ray diffraction experiment, the crystal was removed from solution, covered in Paratone oil, and mounted on a cryoloop (Hampton Research). The mounted crystal was quickly positioned under a cold $\mathrm{N}_{2}$ stream of the Bruker AXS SMART X-ray diffractometer with an APEX-II CCD detector. The data set was recorded as $\omega$-scans at $0.3^{\circ}$ stepwidth and integrated with the Bruker SAINT software package. ${ }^{[\mathrm{S} 1]}$ A multi-scan adsorption correction was applied based on fitting a function to the empirical transmission surface as sampled by multiple equivalent measurements (SADABS). ${ }^{[\mathrm{S} 2]}$ The space group determination was performed with $\mathrm{XPREP},{ }^{[\mathrm{S} 3]}$ while the solution and refinement of the crystal structures were carried out using the SHELX programs. ${ }^{[\mathrm{S} 4]}$ The final refinement was performed with anisotropic atomic displacement parameters for all but $\mathrm{H}$ atoms. All $\mathrm{H}$ atoms were placed in calculated positions. A summary of pertinent information relating to unit cell parameters, data collection, and refinement is provided in Table S1.

Table S1. Data collection and structure refinement parameters for 28.

\begin{tabular}{lc}
\hline Formula & $\mathrm{C}_{23} \mathrm{H}_{25} \mathrm{~N}$ \\
$T, \mathrm{~K}$ & $110(2)$ \\
\hline CCDC number & 1486383 \\
Formula weight & 315.44 \\
Space group & $P \overline{1}$ \\
$a, \AA$ & $7.153(2)$ \\
$b, \AA$ & $10.925(2)$ \\
$c, \AA$ & $12.115(3)$ \\
$\alpha$, deg & $75.115(3)$ \\
$\beta$, deg & $77.430(3)$ \\
$\gamma$, deg & $81.256(3)$ \\
$V, \AA{ }^{3}$ & $888.4(3)$ \\
$Z$ & 2 \\
Crystal color & $c l e a r$ \\
Crystal size, mm & \\
$d_{\text {calc }}$, g cm $^{-3}$ & $0.12 \times 0.04 \times 0.03$ \\
$\mu$, mm & -1 \\
$\lambda, \AA$ & 1.179 \\
$2 \theta_{\text {max }}$, deg & 0.07 \\
Total reflections & 0.71073 \\
$R_{\text {int }}$ & 28.213 \\
Unique reflections $_{\text {Parameters refined }}$ & 3908 \\
Restraints used & 0.0272 \\
\hline
\end{tabular}




$$
\begin{array}{lc}
R_{1}, w R_{2}[I>2 \sigma(I)]^{a} & 0.057,0.155 \\
R_{1}, w R_{2} \text { (all data) } & 0.107,0.189 \\
\text { Goodness of fit }{ }^{b} & 1.027 \\
\text { Diff. peak/hole, e } \AA^{-3} & 0.36,-0.24 \\
\hline{ }^{a} R_{1}=\Sigma|| F_{\mathrm{o}}-\left|F_{\mathrm{c}}\right| / \Sigma\left|F_{\mathrm{o}}\right| ; w R_{2}=\left[\Sigma\left[w\left(F_{\mathrm{o}}{ }^{2}-F_{\mathrm{c}}{ }^{2}\right)^{2}\right] / \Sigma\left[w\left(F_{\mathrm{o}}{ }^{2}\right)^{2}\right]\right]^{1 / 2} \\
{ }^{b} \text { Goodness-of-fit }=\left[\Sigma\left[w\left(F_{\mathrm{o}}{ }^{2}-F_{\mathrm{c}}{ }^{2}\right)^{2}\right] /\left(N_{\mathrm{obs}}-N_{\text {params }}\right]^{1 / 2}\right. \text {, based on all data }
\end{array}
$$

[S1] Bruker, SMART and SAINT; Bruker AXS Inc.: Madison, WI, USA, 2007.

[S2] G. M. Sheldrick $S A D A B S$; University of Gottingen: Gottingen, Germany, 1996.

[S3] G. M. Sheldrick, XPREP. Space group determination and reciprocal space plots.

Siemens Analytical X-ray Instruments: Madison, WI, USA, 1991.

[S4] G. M. Sheldrick, Acta Crystallogr. Sect. A 2008, A64, 112. 